\title{
Study of quark and gluon jet substructure in Z+jet and dijet events from pp collisions
}

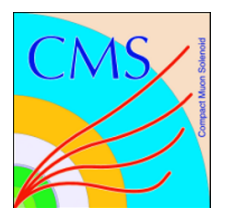

\section{The CMS collaboration}

E-mail: cms-publication-committee-chair@cern.ch

ABSTRACT: Measurements of jet substructure describing the composition of quark- and gluon-initiated jets are presented. Proton-proton (pp) collision data at $\sqrt{s}=13 \mathrm{TeV}$ collected with the CMS detector are used, corresponding to an integrated luminosity of $35.9 \mathrm{fb}^{-1}$. Generalized angularities are measured that characterize the jet substructure and distinguish quark- and gluon-initiated jets. These observables are sensitive to the distributions of transverse momenta and angular distances within a jet. The analysis is performed using a data sample of dijet events enriched in gluon-initiated jets, and, for the first time, a $\mathrm{Z}+$ jet event sample enriched in quark-initiated jets. The observables are measured in bins of jet transverse momentum, and as a function of the jet radius parameter. Each measurement is repeated applying a "soft drop" grooming procedure that removes soft and large angle radiation from the jet. Using these measurements, the ability of various models to describe jet substructure is assessed, showing a clear need for improvements in Monte Carlo generators.

KeYwords: Hadron-Hadron Scattering, Jet Physics

ArXiv ePrint: 2109.03340 


\section{Contents}

1 Introduction 1

2 The CMS detector and event reconstruction 3

3 Data and simulated samples 5

$\begin{array}{lll}4 & \text { Event selection } & 7\end{array}$

5 Jet substructure observables $\quad 10$

6 Unfolding and systematic uncertainties 12

$\begin{array}{lll}7 & \text { Results and discussion } & 15\end{array}$

$\begin{array}{lll}8 & \text { Summary } & 25\end{array}$

$\begin{array}{ll}\text { The CMS collaboration } & 34\end{array}$

\section{Introduction}

Interactions between quarks and gluons, commonly referred to as partons, are governed by quantum chromodynamics (QCD). When quarks or gluons are produced by collisions in particle colliders, collimated showers of hadrons are formed. Theoretical calculations and experimental methods cluster such showers into jets using dedicated clustering algorithms [1] that correlate the kinematic properties of the jets with those of the participating partons. Furthermore, the study of the distribution of particles within a jet, the jet substructure, can be analyzed to determine the likelihood that a jet originates either from a quark or from a gluon. Heavy, Lorentz-boosted particles that decay to quarks and/or gluons, e.g. top quarks or $\mathrm{W}, \mathrm{Z}$ or Higgs bosons, have characteristic distributions of jet substructure observables. Several algorithms have been developed to distinguish such jets from those originating from single partons [2-4]. These are used in numerous measurements and searches at the CERN LHC, and frequently the modelling of jet substructure contributes significantly to the systematic uncertainties [2]. Many of them would benefit from a better understanding and simulation of jet substructure observables.

Although there is no unique way of defining whether a jet originates from a quark or gluon in QCD [5], at leading order (LO) in perturbation theory, one can label jets according to the parton initiating the particle shower. Jets originating from single quarks, quark jets, and those arising from single gluons, gluon jets, are known to differ in their substructure. At LO in perturbative QCD, the difference in the Casimir colour factor between quarks 
$\left(C_{\mathrm{F}}=4 / 3\right)$ and gluons $\left(C_{\mathrm{A}}=3\right)$ leads to a higher probability for gluons to radiate a soft gluon by a factor of $C_{\mathrm{A}} / C_{\mathrm{F}}=9 / 4[6]$. Therefore, we expect gluon jets to contain, on average, more constituents, with a wider geometric spread in the detector [7-12]. However, there are also a variety of other nonperturbative effects that determine the jet substructure, including hadronization and colour reconnection, which are typically described by phenomenological models in Monte Carlo (MC) event generators. The description of jet fragmentation and substructure thus requires a combined understanding of both perturbative calculations and nonperturbative effects. Although the description of jets initiated by quarks is well constrained by many previous measurements, the modelling of gluon jets is less well understood $[2,5]$.

Previous measurements of observables sensitive to jet fragmentation at the LHC have been reported by the ATLAS [13-19], CMS [20-24], ALICE [25-28], and LHCb [29] Collaborations. Jet substructure quantities for the separation of jets initiated by quarks, gluons, W, Z, and Higgs bosons, and top quarks have been measured by ATLAS [30-40], CMS [4145] and ALICE [46, 47]. The goal of this measurement is to systematically explore a set of observables in multiple dimensions of phase space. As outlined in ref. [5], multiple aspects of MC event simulation models of jet substructure can be tested with such a comprehensive set of measurements.

In this paper, we report on a measurement of a set of five observables that can be used to distinguish between quark- and gluon-initiated jets, the generalized angularities $\lambda_{\beta}^{\kappa}$ [48], defined as:

$$
\lambda_{\beta}^{\kappa}=\sum_{i \in \mathrm{jet}} z_{i}^{\kappa}\left(\frac{\Delta R_{i}}{R}\right)^{\beta},
$$

where $z_{i}$ is the fractional transverse momentum carried by the $i$ th jet constituent, $R$ is the jet radius parameter, and $\Delta R_{i}$ is the displacement of the constituent from the jet axis, defined as $\Delta R_{i}=\sqrt{\left(\Delta y_{i}\right)^{2}+\left(\Delta \phi_{i}\right)^{2}}$ where $\Delta y_{i}$ and $\Delta \phi_{i}$ are the separations in rapidity and azimuthal angle (in radians), respectively, between the jet axis and the $i^{\text {th }}$ constituent. The parameters $\kappa \geq 0$ and $\beta \geq 0$ control the momentum and angular contributions, respectively. The five observables measured in this paper are: Les Houches Angularity $($ LHA $)=\lambda_{0.5}^{1}[49] ;$ width $=\lambda_{1}^{1}$; thrust $=\lambda_{2}^{1}[50] ;$ multiplicity $=\lambda_{0}^{0}$; and $\left(p_{\mathrm{T}}^{\mathrm{D}}\right)^{2}=\lambda_{0}^{2}$. These are shown in figure 1 as points in the $(\kappa, \beta)$ plane. These quantities have previously been used by the CMS [51, 52] and ATLAS [53] Collaborations to discriminate between quark and gluon jets. They have also been proposed as a tool to measure parton distribution functions [54] and double-parton scattering [55] in $\mathrm{Z}+$ jets events.

We measure these observables in quark-jet enriched $Z+$ jet and gluon-jet enriched dijet samples. In the $\mathrm{Z}+$ jet sample, we study a jet recoiling against a $\mathrm{Z}$ boson decaying to a pair of muons. The measurement focuses on muons rather than electrons, since effects from bremsstrahlung are negligible for them. Within the dijet event sample, we separately measure the distributions for the more central (smaller absolute rapidity $|y|$ ) of the two jets, and the more forward (larger $|y|$ ) of the two jets. The sample of the more central jets is predicted to provide a higher purity of gluon jets [17]. Each quantity is measured as a function of jet transverse momentum $p_{\mathrm{T}}$, with different jet radius parameters, including all 


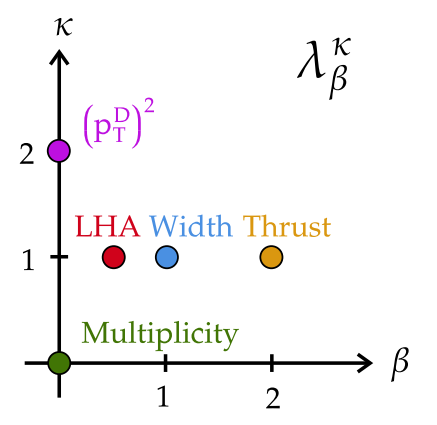

Figure 1. The five generalized angularities $\lambda_{\beta}^{\kappa}$ used in this analysis, represented in the $(\kappa, \beta)$ plane. The Les Houches Angularity is denoted by LHA. Adapted from ref. [48].

particles and only charged particles, and with and without applying a soft drop grooming procedure [56] that removes soft and wide angle radiation from the jet, thereby making the jet substructure observables more resilient to effects from pileup, underlying event, and initial state radiation. The detector level distributions are unfolded to particle level, i.e. only considering final state particles after hadronization with proper lifetime $c \tau>$ $10 \mathrm{~mm}$, for each configuration and observable, and normalized to unity in each $p_{\mathrm{T}}$ bin. The measurements are finally compared with predictions based on analytic resummation [57].

After a brief description of the CMS detector in section 2, details about the data and simulated samples are given in section 3, followed by a description of the event selection in section 4 . The jet substructure observables are then more precisely introduced in section 5 . The unfolding procedure and uncertainties of the measurement are described in section 6 . Finally, results are presented and discussed in section 7 , and briefly summarized in section 8 .

\section{The CMS detector and event reconstruction}

The central feature of the CMS apparatus is a superconducting solenoid of $6 \mathrm{~m}$ internal diameter, providing a magnetic field of $3.8 \mathrm{~T}$. Within the solenoid volume are a silicon pixel and strip tracker, a lead tungstate crystal electromagnetic calorimeter (ECAL), and a brass and scintillator hadron calorimeter (HCAL), each composed of a barrel and two endcap sections. Forward calorimeters extend the pseudorapidity $(\eta)$ coverage provided by the barrel and endcap detectors. Muons are detected in gas-ionization chambers embedded in the steel flux-return yoke outside the solenoid.

A more detailed description of the CMS detector, together with a definition of the coordinate system used and the relevant kinematic variables, can be found in ref. [58].

The silicon tracker measures charged particles within the range $|\eta|<2.5$. It consists of 1440 silicon pixel and 15148 silicon strip detector modules. For particles of $1<p_{\mathrm{T}}<$ $10 \mathrm{GeV}$ and $|\eta|<1.4$, the track resolutions are typically $1.5 \%$ in $p_{\mathrm{T}}$ and $25-90(45-150) \mu \mathrm{m}$ in the transverse (longitudinal) impact parameter [59].

The candidate vertex with the largest value of summed physics-object $p_{\mathrm{T}}^{2}$ is the primary proton-proton $(\mathrm{pp})$ interaction vertex. The physics objects are jets, clustered using the 
anti- $k_{\mathrm{T}}$ jet finding algorithm $[60,61]$ with the tracks assigned to a candidate vertex as inputs, and the associated missing transverse momentum, which is the negative vector sum of the $p_{\mathrm{T}}$ of those jets.

Muons are measured in the pseudorapidity range $|\eta|<2.4$, with detection planes made using three technologies: drift tubes, cathode strip chambers, and resistive plate chambers. The single muon trigger efficiency exceeds $90 \%$ over the full $\eta$ range, and the efficiency to reconstruct and identify muons is greater than $96 \%$. Matching muons to tracks measured in the silicon tracker results in a relative transverse momentum resolution, for muons with $p_{\mathrm{T}}$ up to $100 \mathrm{GeV}$, of $1 \%$ in the barrel and $3 \%$ in the endcaps. The $p_{\mathrm{T}}$ resolution in the barrel is better than $7 \%$ for muons with $p_{\mathrm{T}}$ up to $1 \mathrm{TeV}[62]$.

The particle-flow algorithm [63] is used to reconstruct and identify each individual particle in an event, with an optimized combination of information from the various elements of the CMS detector. The energy of photons is obtained from the ECAL measurement. The energy of electrons is determined from a combination of the electron momentum at the primary interaction vertex as determined by the tracker, the energy of the corresponding ECAL cluster, and the energy sum of all bremsstrahlung photons spatially compatible with originating from the electron track. The energy of muons is obtained from the curvature of the corresponding track. The energy of charged hadrons is determined from a combination of their momentum measured in the tracker and the matching ECAL and HCAL energy deposits, corrected for the response function of the calorimeters to hadronic showers. Finally, the energy of neutral hadrons is obtained from the corrected ECAL and HCAL energies.

For each event, hadronic jets are clustered from these reconstructed particles using the infrared and collinear (IRC) safe anti- $k_{\mathrm{T}}$ algorithm [60, 61] with distance parameters of 0.4 and 0.8 , referred to as AK4 and AK8 jets in the following. Jet momentum is determined as the vectorial sum of all particle momenta in the jet. It is found from simulation to be, on average, within 5 to $10 \%$ of the true momentum over the whole $p_{\mathrm{T}}$ spectrum and detector acceptance.

Additional pp interactions within the same or nearby bunch crossings (pileup) can contribute additional tracks and calorimetric energy depositions to the jet momentum. The pileup per particle identification algorithm $[64,65]$ is used to mitigate the effect of pileup at the level of reconstructed particles, making use of the local momentum distribution, event pileup properties and tracking information. For each neutral particle, a momentum distribution variable $\log \sum p_{\mathrm{T}}^{2} / \Delta R^{2}$ is computed using the surrounding charged particles compatible with the primary vertex within the tracker acceptance $(|\eta|<2.5)$, and using both charged and neutral particles in the region outside of the tracker coverage, where $p_{\mathrm{T}}$ and $\Delta R$ correspond to the transverse momenta and distances of the surrounding particles in the $\eta$ - $\phi$ plane w.r.t. the particle direction. The probability to originate from the primary interaction vertex is deduced by comparing the momentum distribution variable to its event median characterizing the expected value for particles from pileup vertices. The momenta of the neutral particles are rescaled according to their probability, superseding the need for jet-based pileup corrections [65]. Charged particles identified to be originating from pileup vertices are discarded. 
Jet energy corrections are derived from simulation to adjust the measured response of jets to that of particle level jets on average. In situ measurements of the momentum balance in dijet, photon + jet, $\mathrm{Z}+$ jet, and multijet events are used to account for any residual differences in jet energy scale in experimental data and simulation [66]. The jet energy resolution amounts typically to $15 \%$ at $10 \mathrm{GeV}, 8 \%$ at $100 \mathrm{GeV}$, and $4 \%$ at $1 \mathrm{TeV}$. Additional selection criteria are applied to each jet to remove jets potentially dominated by anomalous contributions from various subdetector components or reconstruction failures [65].

Events of interest are selected using a two-tiered trigger system [67]. The first level, composed of custom hardware processors, uses information from the calorimeters and muon detectors to select events at a rate of around $100 \mathrm{kHz}$ within a fixed latency of about $4 \mu \mathrm{s}$ [68]. The second level, known as the high-level trigger, consists of a farm of processors running a version of the full event reconstruction software optimized for fast processing, and reduces the event rate to around $1 \mathrm{kHz}$ before data storage.

\section{Data and simulated samples}

The analysis is based on pp collision data collected by the CMS experiment in 2016 at a centre-of-mass energy of $\sqrt{s}=13 \mathrm{TeV}$, corresponding to an integrated luminosity of $35.9 \mathrm{fb}^{-1}$ and with an average of 23 interactions per bunch crossing. Events are selected by the trigger system using multiple sets of trigger paths. The $\mathrm{Z}+$ jet candidate events are collected with a trigger requiring at least one isolated muon with $p_{\mathrm{T}}>24 \mathrm{GeV}$ and $|\eta|<2.4$; the efficiency to select an event with at least one muon exceeds 90\% [69]. Dijet events with high- $p_{\mathrm{T}}$ jets are collected with triggers requiring at least one AK4 or AK8 jet above a certain threshold in $p_{\mathrm{T}}$ with an efficiency exceeding 99\%. The triggers with $p_{\mathrm{T}}<450 \mathrm{GeV}$ are "prescaled", i.e. only a fraction of the collision events passing the trigger requirements are accepted, which results in a lower integrated luminosity for these triggers. Dijet events with jet $p_{\mathrm{T}}<40 \mathrm{GeV}$ are collected with a zero bias trigger that randomly selects a fraction of the collision events. Table 1 summarizes the zero bias and jet triggers with their integrated luminosity and the offline $p_{\mathrm{T}}$ bin thresholds for which the triggers are used.

The processes of interest in this analysis are the $\mathrm{Z}$ boson production in association with jets, and events uniquely composed of jets produced through the strong interaction (QCD multijet production). Their simulation is performed with multiple combinations of $\mathrm{MC}$ event generators to estimate the detector response and systematic uncertainties. The $\mathrm{Z}$ boson production in association with jets is simulated at LO with the MADGRAPH5_aMC@NLO v2.2.2 [70] generator with up to four outgoing partons in the matrix element calculations, and fragmented with PYтніA v8.212 [71]. PYTHIA8 implements a dipole shower ordered in $p_{\mathrm{T}}$ and the hadronization of quarks and gluons into final hadrons is described by the Lund string model $[72,73]$. Double counting of jets from the matrix element calculation and parton shower is eliminated using the MLM merging scheme [74]. This sample is hereafter referred to by MG5+PYTHIA8. The PYTHIA 8 parameters for the underlying event are set according to the CUETP8M1 tune $[75,76]$. A second $\mathrm{Z}+$ jet sample is generated at LO with HERWIG ++ v2.7.1 $[77,78]$ in standalone mode with the UE-EE-5C underlying event tune [79] to assess systematic uncertainties related to the modelling of the 


\begin{tabular}{|cccc|}
\hline $\begin{array}{c}\text { Online } p_{\mathrm{T}} \text { threshold } \\
(\mathrm{GeV})\end{array}$ & $\begin{array}{c}\text { Integrated luminosity } \\
\left(\mathrm{fb}^{-1}\right)\end{array}$ & $\begin{array}{c}\text { Offline } p_{\mathrm{T}} \text { bin threshold(s) } \\
(\mathrm{GeV})\end{array}$ & Number of events \\
\hline 0 (zero bias) & $2.90 \times 10^{-5}$ & 50 & 309512 \\
40 & $2.64 \times 10^{-4}$ & 65 & 446183 \\
60 & $7.19 \times 10^{-4}$ & 88 & 387849 \\
80 & $2.73 \times 10^{-3}$ & 120,150 & 757778 \\
140 & $2.39 \times 10^{-2}$ & 186 & 1014702 \\
200 & $1.03 \times 10^{-1}$ & 254 & 841507 \\
260 & $5.88 \times 10^{-1}$ & 326 & 1821727 \\
320 & 1.75 & 408 & 1918614 \\
400 & 5.14 & 481 & 1960323 \\
450 & 35.9 & $614,800,1000$ & 6421782 \\
\hline
\end{tabular}

Table 1. Summary of zero bias and jet triggers used in the analysis for the dijet region. For each trigger, the integrated luminosity and number of events collected by it are given. The offline $p_{\mathrm{T}}$ bin threshold(s) indicate the lower edge of the $p_{\mathrm{T}} \operatorname{bin}(\mathrm{s})$ measured with data from a given trigger.

parton showering and hadronization. In HERWIG ++ , the parton shower follows angularordered radiation [80], and the hadronization is described by the cluster model [81]. This sample has one outgoing parton in the matrix element calculations, recoiling against the Z boson. The MADGRAPH5_aMC@NLO and HERWIG++ samples are normalized to the differential cross sections at next-to-LO (NLO) in electroweak and next-to-NLO (NNLO) in strong interactions [82].

The QCD multijet production is simulated separately with two different generator configurations: MADGRAPH5_amC@NLO combined with PYTHIA8, and HERWIG ++ in a standalone mode. In the former sample, again referred to by MG5+PYTHIA8, up to four outgoing partons are allowed in the matrix element calculations using the MLM jet merging scheme. In the standalone HERWIG ++ sample, the matrix element has two outgoing partons, and the same underlying event tune is used as in the HERWIG $++\mathrm{Z}+$ jet sample. The LO NNPDF 3.0 [83] parton distribution functions (PDFs) with $\alpha_{\mathrm{S}}\left(m_{\mathrm{Z}}\right)=0.130$ are used in all generated samples, where $\alpha_{\mathrm{S}}$ is the strong coupling and $m_{\mathrm{Z}}$ is the $\mathrm{Z}$ boson mass. Backgrounds from top quark-antiquark pair production, $\mathrm{W}$ boson production in association with jets, as well as diboson production, are estimated to contribute less than $1 \%$ to the analysis phase space from MC and are not included.

All generated samples are passed through a detailed simulation of the CMS detector using Geant4 [84]. To simulate the effect of pileup, multiple inelastic events are generated using PYTHIA8 and superimposed on the primary interaction events. The MC simulated events are scaled to reproduce the distribution of the number of interactions observed in the experimental data. The MG5+PYTHIA8 and HERWIG ++ simulations have additional selection requirements to ensure that the energy scale of the generated primary interaction events is greater than those of the overlapping pileup events. This can affect the $\mathrm{MC} p_{\mathrm{T}}$ distribution slope at low $p_{\mathrm{T}}$, within the total MC uncertainty; however, this has a negligible effect on the unfolded results and uncertainties. 
Additional samples of $\mathrm{Z}$ boson production in association with jets and QCD multijet production, without detector simulation, are compared with the unfolded experimental data distributions. Predictions are computed at LO with PYTHIA v8.243 with the CP2 and CP5 tunes [85], HERWIG v7.1.4 [86] at LO with angular-ordered showering and the CH3 tune [87], and with SHERPA v2.2.10 [88-90] at LO, and also with two (one) extra jets for QCD multijet $(\mathrm{Z}+\mathrm{jet})$ production with the CKKW matching procedure [91-93]. The parton shower in SHERPA is based on the Catani-Seymour dipole factorization [94], and hadrons are formed by a modified cluster hadronization model [95].

As discussed in refs. $[41,85,87,96]$, the choice of $\alpha_{\mathrm{S}}\left(m_{\mathrm{Z}}\right)$ used in the final-state shower evolution in MC event generators has a significant impact on the shape of jet substructure observables for quark and gluon jets. Whereas the MG5+PYTHIA8, HERWIG++, and PYTHIA8 CP2 predictions use larger values of $\alpha_{\mathrm{S}}\left(m_{\mathrm{Z}}\right)$ for the final-state showering of $0.1383,0.127$, and 0.130 , respectively, the PYTHIa8 CP5, HERWIG CH3, and SHERPA predictions all use a common value of 0.118. The PYTHIA8 CP2 and PYTHIA8 CP5 tunes differ mainly in their choice of $\alpha_{\mathrm{S}}\left(m_{\mathrm{Z}}\right)$ that is also used in the underlying event model. They were obtained by tuning the other parameters controlling the underlying event in PYTHIA8 (except $\alpha_{\mathrm{S}}\left(m_{\mathrm{Z}}\right)$ ) to describe LHC data [85]. Their comparison thus reflects the difference due to the choice of $\alpha_{\mathrm{S}}\left(m_{\mathrm{Z}}\right)$, while keeping a good description of the underlying event. In addition to the choice of $\alpha_{\mathrm{S}}\left(m_{\mathrm{Z}}\right)$, the shapes of jet substructure observables are sensitive to the description of gluon splitting to quarks and colour reconnection in the final state [5].

\section{Event selection}

The measurement of the generalized angularities (figure 1) is carried out using two event samples: a Z+jet sample that is enriched in quark jets, and a dijet sample that is enriched in gluon jets. To calculate each angularity for AK4 and AK8 jets, the following event selection is carried out with the AK4 and AK8 jet collections, respectively. The $\mathrm{Z}+$ jet selection requires at least two reconstructed muons with $p_{\mathrm{T}}>26 \mathrm{GeV}$, which results in a trigger efficiency exceeding $99 \%$. A Z boson candidate is reconstructed from two oppositelycharged muons with $|\eta|<2.4$ and a dimuon invariant mass within $20 \mathrm{GeV}$ of $m_{\mathrm{Z}}$, requiring $p_{\mathrm{T}}^{\mathrm{Z}}>30 \mathrm{GeV}$. At least one reconstructed jet with $p_{\mathrm{T}}>30 \mathrm{GeV}$ and $|y|<1.7$ that does not overlap with the muons of the $\mathrm{Z}$ boson candidate $\left(\Delta R\left(\mathrm{j}_{1}, \mu_{1,2}\right)>R=0.4\right.$ or 0.8 for AK4 or AK8 jets, respectively) is required. The requirement on the jet rapidity ensures that jets are fully within the tracker coverage. The $\mathrm{Z}$ boson candidate with transverse momentum $p_{\mathrm{T}}^{\mathrm{Z}}$, and the jet with the largest $p_{\mathrm{T}}$, with transverse momentum $p_{\mathrm{T}}^{\mathrm{j}_{1}}$, are required to have a $p_{\mathrm{T}}$ asymmetry $\left|p_{\mathrm{T}}^{\mathrm{j}_{1}}-p_{\mathrm{T}}^{\mathrm{Z}}\right| /\left(p_{\mathrm{T}}^{\mathrm{j}_{1}}+p_{\mathrm{T}}^{\mathrm{Z}}\right)<0.3$, and an azimuthal separation $\Delta \phi\left(\mathrm{j}_{1}, \mathrm{Z}\right)>2$ radians. The requirements on $p_{\mathrm{T}}$ asymmetry and azimuthal separation are introduced to restrict the measurement to a phase space dominated by two-body scattering where a single parton is produced together with the $\mathrm{Z}$ boson in the hard scattering process. In this phase space large contributions from higher order QCD corrections, arising when $p_{\mathrm{T}}^{\mathrm{j}_{1}}$ is significantly larger than $p_{\mathrm{T}}^{\mathrm{Z}}$ [97], are suppressed. The substructure of this jet is measured. 


\begin{tabular}{|ll|}
\hline Sample & Event selection \\
\hline Z+jet & $\geq 2$ muons with $|\eta|<2.4$ and $p_{\mathrm{T}}^{\mu}>26 \mathrm{GeV}$ \\
& Opposite-charge muons \\
& $\left|m_{\mu \mu}-m_{\mathrm{Z}}\right|<20 \mathrm{GeV}$ \\
& $p_{\mathrm{T}}^{\mathrm{Z}}>30 \mathrm{GeV}$ \\
& $\geq 1$ jet with $|y|<1.7$ and $p_{\mathrm{T}}^{\mathrm{j}}>30 \mathrm{GeV}$, \\
& $\Delta R\left(\mathrm{j}_{1}, \mu_{1,2}\right)>R=0.4$ or 0.8 \\
& $\Delta \phi\left(\mathrm{j}_{1}, \mathrm{Z}\right)>2$ \\
& $\left|p_{\mathrm{T}}^{\mathrm{j}_{1}}-p_{\mathrm{T}}^{\mathrm{Z}}\right| /\left(p_{\mathrm{T}}^{\mathrm{j}_{1}}+p_{\mathrm{T}}^{\mathrm{Z}}\right)<0.3$ \\
& $\geq 2$ jets with $|y|<1.7$ and $p_{\mathrm{T}}^{\mathrm{j}}>30 \mathrm{GeV}$ \\
Dijet & $\Delta \phi\left(\mathrm{j}_{1}, \mathrm{j}_{2}\right)>2$ \\
(central and & $\left|p_{\mathrm{T}}^{\mathrm{j}_{1}}-p_{\mathrm{T}}^{\mathrm{j}_{2}}\right| /\left(p_{\mathrm{T}}^{\mathrm{j}_{1}}+p_{\mathrm{T}}^{\mathrm{j}_{2}}\right)<0.3$ \\
forward) & \\
\hline
\end{tabular}

Table 2. Summary of the selection criteria for the $\mathrm{Z}+$ jet and dijet event samples.

The dijet selection requires at least two reconstructed jets with $p_{\mathrm{T}}>30 \mathrm{GeV}$ and $|y|<1.7$. The two jets with largest transverse momentum, $p_{\mathrm{T}}^{\mathrm{j}_{1,2}}$, are required to have a transverse momentum asymmetry $\left|p_{\mathrm{T}}^{\mathrm{j}_{1}}-p_{\mathrm{T}}^{\mathrm{j}_{2}}\right| /\left(p_{\mathrm{T}}^{\mathrm{j}_{1}}+p_{\mathrm{T}}^{\mathrm{j}_{2}}\right)<0.3$, and an azimuthal separation larger than 2 radians. The angularities are calculated for these two jets. In the following, the jet with smaller $|y|$ is labelled as the central jet, whereas the jet with larger $|y|$ is labelled as the forward jet.

Table 2 summarizes the selection criteria of the $\mathrm{Z}+$ jet and dijet event samples. The detector-level distributions are unfolded to particle-level, i.e. only considering final-state particles. At particle level the same selection criteria are applied except that the muons of the $\mathrm{Z}$ boson candidate are removed from the particles used as input to the jet clustering, instead of requiring $\Delta R\left(\mathrm{j}_{1}, \mu_{1,2}\right)>R$. Muons at particle level are not "dressed", i.e. radiated photons are not considered part of the muon candidate, since this has a negligible effect on the muons. Although the detector resolution of jet $|y|$ and $\phi$, as well as that of the muon $p_{\mathrm{T}}$, is small enough to maintain equivalent particle- and detector-level selection criteria, the resolution of jet $p_{\mathrm{T}}$ is significantly worse. The measurement is defined for jets with $p_{\mathrm{T}}>50 \mathrm{GeV}$ at particle level. However, because of resolution effects, jets with $p_{\mathrm{T}} \sim 50 \mathrm{GeV}$ at particle level can migrate to lower transverse momentum bins at detector level. The unfolding procedure is therefore carried out for jets with $p_{\mathrm{T}}>30 \mathrm{GeV}$ to minimise modelling uncertainties associated with such migrations.

We quantify the fraction of quark and gluon jets in these event samples using the MG5+PYTHIA8 simulation. As discussed in ref. [5], there is no unique way of defining a quark or gluon jet, and our choice of approach is as follows. The parton from which a jet originates is obtained by first clustering jets from all generated partons (from the matrix element and the shower up to hadronization) together with the generated finalstate particles, where the four-momenta of the generated partons are scaled by a very 


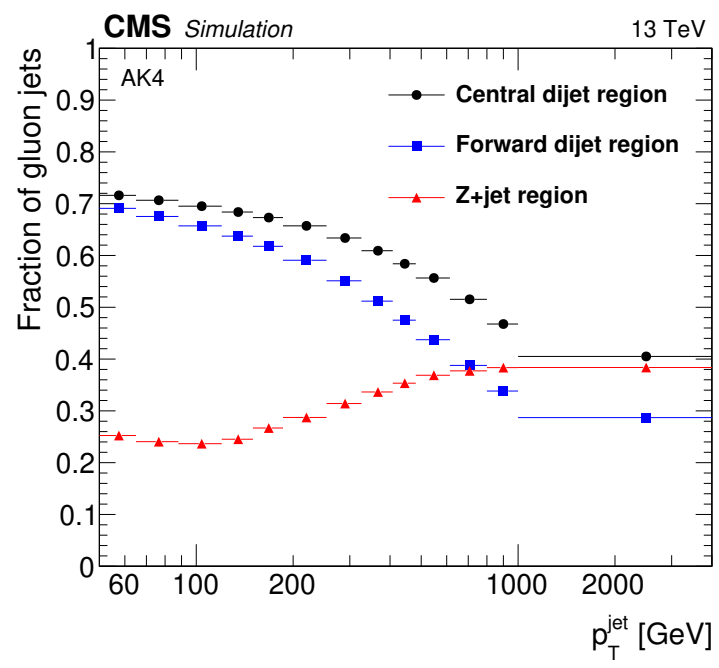

Figure 2. Fraction of AK4 jets that are gluon jets in the $\mathrm{Z}+$ jet sample (red triangles), and the central (black circles) and forward (blue squares) dijet samples. The statistical uncertainty is negligible in the simulation.

small number. The jets clustered in this way are almost identical to those clustered without generator partons because the added partons, with extremely small energy, do not affect the clustered jet momentum. The quark or gluon with highest $p_{\mathrm{T}}$ in the jet then defines the origin of the jet. Figure 2 shows the resulting fraction of AK4 jets which are gluon jets in the $\mathrm{Z}+$ jet sample, and central and forward dijet samples as a function of jet $p_{\mathrm{T}}$. The $\mathrm{Z}+$ jet sample is enriched in quark jets, making up $64-76 \%$ of the jets in the sample. The central and forward dijet samples are dominated by $69-72 \%$ gluon jets at lowest $p_{\mathrm{T}}$, and by $55-68 \%$ quark jets at highest $p_{\mathrm{T}}$. The central dijet jets have a consistently higher fraction of gluon jets than the forward dijet jets across the entire $p_{\mathrm{T}}$ range. The fraction of gluon jets was also computed with HERWIG++, PYTHIA8 CP5, PYTHIA8 CP2, HERWIG7 CH3 and SHERPA. Although the gluon jet fraction in all generators agrees with MG5+PYTHIA 8 within $6 \%$ in the dijet samples, up to $25 \%$ lower gluon fractions are predicted in the $\mathrm{Z}+$ jet sample. The spread reflects the rather large uncertainties associated with LO MC generator predictions.

In figure 2, we consider all quark flavours, except for top, to identify quark jets. Typically, however, quark jets are considered to be jets initiated only by light-flavour quarks $(\mathrm{u}, \mathrm{d}, \mathrm{s})$. The $\mathrm{Z}+$ jet and dijet samples also have a small contribution of $5-15 \%$ from jets initiated by heavy-flavour quarks (b,c), whose behaviour was measured in ref. [41], and is in between that of light-flavour quark jets and gluon jets. Their presence will therefore reduce the expected differences between jets from the $\mathrm{Z}+$ jet and dijet samples.

The $p_{\mathrm{T}}$ distribution in the CMS data and simulation for the $\mathrm{Z}+$ jet and central dijet AK4 jets is shown in figure 3 . The data distribution in bins of jet $p_{\mathrm{T}}$ collected with prescaled triggers (see table 1 ) is multiplied by the corresponding prescale factors to reproduce the smoothly falling physical spectrum. The data distribution is compared with predictions from two generator configurations, where the total number of events in the simulations has 

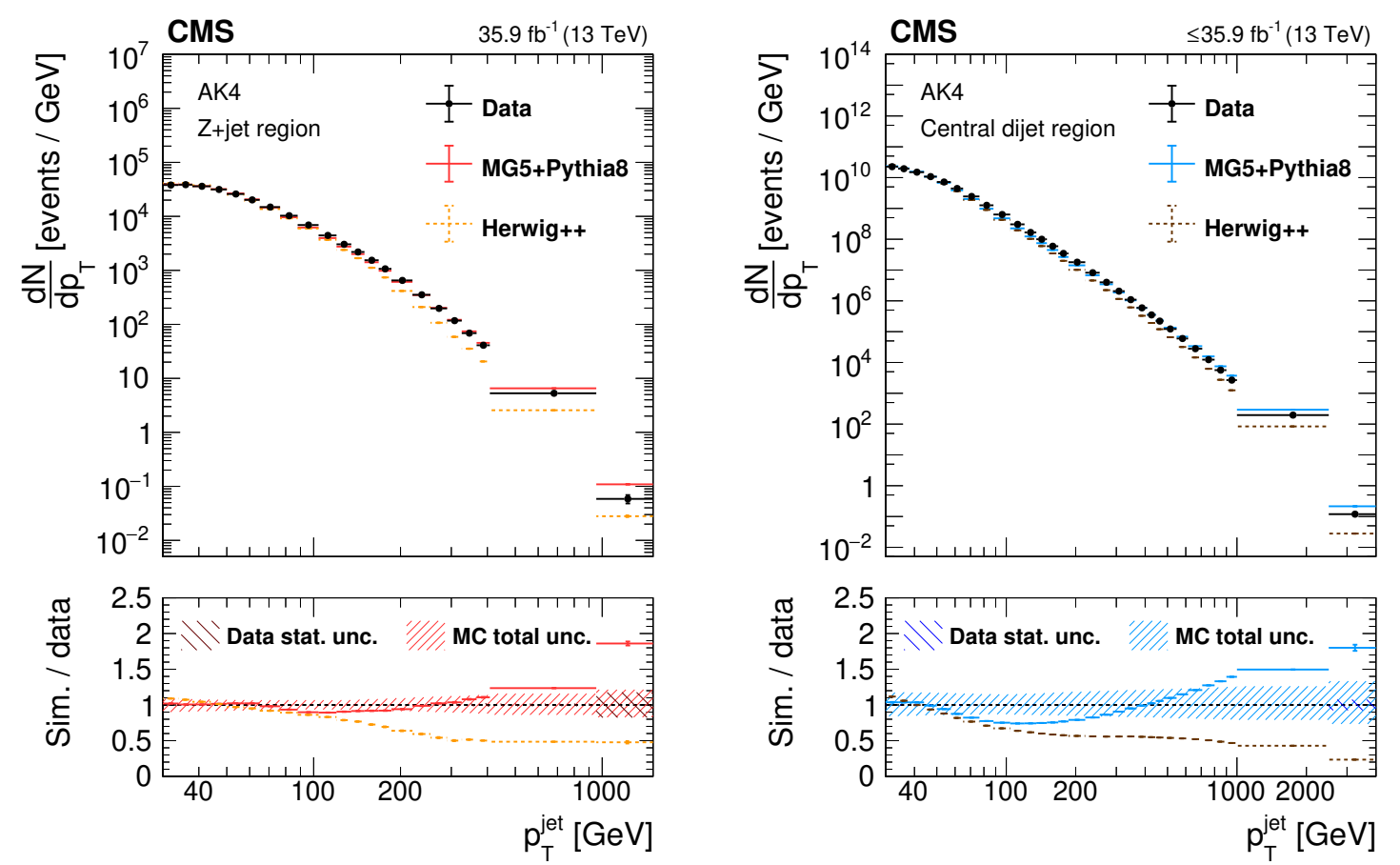

Figure 3. Data to simulation comparisons of the jet $p_{\mathrm{T}}$ in the $\mathrm{Z}+$ jet (left) and central dijet (right) regions. The error bars correspond to the statistical uncertainties in the experimental data. The coarse-grained hashed region in each ratio plot indicates the statistical uncertainty in the experimental data, and the fine-grained hashed region represents the total uncertainty in the MC prediction.

been scaled to match the data distribution. In both selection regions, the MG5+PYTHIA8 simulation provides a better description of the experimental data, and is therefore used to estimate the detector response consistently in both samples in this paper, whereas HERWIG ++ is used to evaluate the systematic uncertainties. The disagreements observed in the $\mathrm{Z}+$ jet and central dijet $p_{\mathrm{T}}$ distributions indicate a sensitivity to higher order corrections not fully modelled in these two generators. These differences have, however, a smaller effect on the jet substructure distributions that are measured in bins of jet $p_{\mathrm{T}}$.

\section{$5 \quad$ Jet substructure observables}

For the AK4 and AK8 jets of interest, we compute and measure five generalized angularities as defined in Equation 1.1 that discriminate quark and gluon jets. Of these variables, only LHA, width, and thrust are IRC-safe as a result of having $\kappa=1$ [98]. However, multiplicity and $\left(p_{\mathrm{T}}^{\mathrm{D}}\right)^{2}$ have been widely used for quark and gluon discrimination $[51,52]$. For $\beta>1$, the anti- $k_{\mathrm{T}}$ jet axis is used to calculate $\Delta R$. For $\beta \leq 1, \Delta R$ is calculated using the jet axis constructed by the winner-takes-all (WTA) method [98]. The use of the WTA axis significantly simplifies theoretical calculations of the angularities, whereas for angularities with $\beta>1$ computation is feasible with both axis definitions. However, using the anti- $k_{\mathrm{T}}$ jet axis results in an observable that is more akin to the jet mass [42]. To calculate the WTA axis, 
the constituents of the anti- $k_{\mathrm{T}}$ jet are reclustered using the $p_{\mathrm{T}}$-based WTA scheme. The resultant jet has the $(y, \phi)$ of the constituent with the largest $p_{\mathrm{T}}$. The calculation of multiplicity includes only constituents with $p_{\mathrm{T}}>1 \mathrm{GeV}$, at both the detector and particle levels.

Each of the angularities is designed to emphasize a particular feature of the jet. The LHA, width, and thrust include both the momentum fraction and the angular distribution of the constituents within the jet. Since the weighting of the angular distribution differs across these variables, comparing them can highlight differences in the constituent positions within the jet. We expect gluon jets to have, on average, larger values of these angularities due to the larger number of constituents that are further from the jet axis. In contrast, $\left(p_{\mathrm{T}}^{\mathrm{D}}\right)^{2}$ places more value on those higher-momentum constituents, irrespective of their position in the jet. With gluon jets typically having more lower-momentum constituents, we therefore expect them to generally have smaller values of $\left(p_{\mathrm{T}}^{\mathrm{D}}\right)^{2}$. Whereas the three IRC-safe angularities are particularly sensitive to the modelling of perturbative emissions in jets, the other two have larger contributions from nonperturbative effects and are thus subject to larger uncertainties in their predictions and measurements.

In this paper, we measure each substructure observable with various configurations. Each quantity is computed in multiple bins of jet $p_{\mathrm{T}}$, and for two different jet radius parameters, $R=0.4$ and 0.8 . For each quantity, we define a variant where the observables is calculated using only the charged constituents in anti- $k_{\mathrm{T}}$ algorithm ("charged"). While observables computed with both charged and neutral constituents can be described more easily from first-principle calculations, the charged variants can be measured with a better resolution as a result of the high efficiency and precision of the tracking detector.

Additionally, we compute a groomed variant of each observable, where the jet is reclustered with the Cambridge-Aachen (CA) algorithm [99], and then groomed using the modified mass-drop algorithm [100, 101], known as the soft drop algorithm [56]. This splits the jet into two subjets by undoing the last step of the CA jet clustering. It regards the jet as the final soft drop jet if the two subjets satisfy the condition:

$$
\frac{\min \left(p_{\mathrm{T}}^{(1)}, p_{\mathrm{T}}^{(2)}\right)}{p_{\mathrm{T}}^{(1)}+p_{\mathrm{T}}^{(2)}}>z_{\text {cut }}\left(\frac{\Delta R_{12}}{R}\right)^{\beta_{\mathrm{sd}}}
$$

where $p_{\mathrm{T}}^{(1)}$ and $p_{\mathrm{T}}^{(2)}$ are the transverse momenta of the two subjets, $R$ is the jet radius parameter, $\Delta R_{12}=\sqrt{\left(\Delta y_{12}\right)^{2}+\left(\Delta \phi_{12}\right)^{2}}$ is the distance between the two subjets, and $z_{\text {cut }}$ and $\beta_{\text {sd }}$ are tunable parameters of the soft drop algorithm, set to $z_{\text {cut }}=0.1$ and $\beta_{\text {sd }}=0$ in this study. If the condition is not met, the subjet with the lower $p_{\mathrm{T}}$ is rejected. The declustering procedure is repeated splitting the higher $p_{\mathrm{T}}$ subjet into two subjets by undoing another CA clustering step, iterating until the condition is met. This grooming procedure removes soft and wide-angle radiation from the jet, thereby making the jet substructure observables more resilient to effects from pileup, underlying event, and initial-state radiation. In all cases, the jet $p_{\mathrm{T}}$ used for binning is taken from the original ungroomed anti- $k_{\mathrm{T}}$ jet (i.e. using charged+neutral constituents, before calculating the WTA axis, and before grooming). A summary of all variations of the observables and the $p_{\mathrm{T}}$ ranges measured in this paper is given in table 3 . Whereas the measurements are carried out for all variations 


\begin{tabular}{|lc|}
\hline Dimension & Variants \\
\hline Region & Z+jet vs. central dijet vs. forward dijet \\
Observable $\lambda_{\beta}^{\kappa}$ & LHA, width, thrust, multiplicity, $\left(p_{\mathrm{T}}^{\mathrm{D}}\right)^{2}$ \\
Jet $p_{\mathrm{T}}$ & $50<p_{\mathrm{T}}<65 \mathrm{GeV}, \ldots, p_{\mathrm{T}}>408 \mathrm{GeV}$ for $\mathrm{Z}+$ jet \\
& $50<p_{\mathrm{T}}<65 \mathrm{GeV}, \ldots, p_{\mathrm{T}}>1000 \mathrm{GeV}$ for dijet \\
Jet radius parameter $R$ & 0.4 vs. 0.8 \\
Constituents & Charged + neutral vs. charged \\
Grooming & Ungroomed vs. groomed \\
\hline
\end{tabular}

Table 3. Summary of the variants of observables measured in this paper.

of the observables and made public in HEPData record [102], this paper will focus on representative distributions, typically taking the ungroomed charged+neutral distribution with $R=0.4$ at $120<p_{\mathrm{T}}<150 \mathrm{GeV}$ as a reference for comparisons. This $p_{\mathrm{T}}$ range is chosen as a compromise between lowest $p_{\mathrm{T}}$ with highest enrichment in gluons for the dijet central sample and higher $p_{\mathrm{T}}$ yielding lower statistical uncertainties for the dijet sample (see table 1) and lower systematic uncertainties because of better jet energy resolutions.

\section{$6 \quad$ Unfolding and systematic uncertainties}

The experimental data distributions are unfolded to particle level using unregularized unfolding as implemented in the TUNFOLD package [103]. The particle-level distribution and covariance matrix are obtained by minimizing $\chi_{A}^{2}=(y-A x)^{T} V_{y y}^{-1}(y-A x)$, where $A$ is the particle-to-reconstructed phase space migration matrix, $V_{y y}$ is an estimate of the variance of the observations $y$, and $x$ is an estimator of the true particle-level values. Here, $V_{y y}$ is a diagonal matrix formed by the square of the bin errors. The migration matrix in the $2 \mathrm{D}$ phase space of $\left(p_{\mathrm{T}}, \lambda_{\beta}^{\kappa}\right)$ is derived from the MG5+PYTHIA8 simulation, matching particle-level jets with detector-level jets by requiring an angular separation of less than half of the jet radius parameter. Before the detector-level distribution is unfolded, background from misreconstructed jets, i.e. jets that pass the reconstruction criteria, but fail the particle-level selection, are subtracted. The proportion of misreconstructed jets ranges from $<10 \%$ for jets at $50 \mathrm{GeV}$ to $<1 \%$ at $1 \mathrm{TeV}$. The contribution from other standard model processes was negligible. The migration matrix also includes those particle-level jets that fail the reconstruction criteria, to correct for the reconstruction inefficiency.

The binning of the migration matrix includes the detector resolution. We define purity as the fraction of reconstructed events that are generated in the same $\left(p_{\mathrm{T}}, \lambda_{\beta}^{\kappa}\right)$ bin, and stability as the fraction of generated events that are reconstructed in the same bin. Both quantities are $\geq 50 \%$ in most bins. The detector-level bins have half the width of the particle-level bins in both $p_{\mathrm{T}}$ and $\lambda_{\beta}^{\kappa}$, to guarantee an over constrained system for the minimization carried out during unfolding.

Various sources of systematic uncertainty are considered, which are treated as uncorrelated among each other, but correlated in the $\left(p_{\mathrm{T}}, \lambda_{\beta}^{\kappa}\right)$ plane. These are divided into 
experimental sources, uncertainties related to the theoretical model used to derive the migration matrix, and inherent unfolding uncertainties. Experimental and physics model uncertainties are treated by constructing variations in the migration matrix, and propagating the changes to the final unfolded $1 \mathrm{D}$ distribution.

Uncertainties in the calibration of the jet energy scale (JES) and jet energy resolution (JER) measurement [66] are included by varying the jet $p_{\mathrm{T}}$ when constructing the migration matrix. Furthermore, we account for variations in the measurement of the particle-flow candidates, which are propagated to the computation of the jet substructure observables in the migration matrix. These include variations of the charged-hadron momentum scale by $\pm 1 \%$, photon energy scale by $\pm 1 \%$, and neutral-hadron energy scale by $\pm 3 \%[63,66]$ that are estimated from single-particle response measurements [104]. We find uncertainties related to the angular resolution of the particle-flow reconstruction and charged-hadron reconstruction efficiency and momentum resolution are negligible. The simulation of pileup is tuned to match the particle multiplicities and $p_{\mathrm{T}}$ spectra observed in data with a dedicated MC tune [85]. We estimate the uncertainty in jet $p_{\mathrm{T}}$ and substructure observables related to pileup by reweighting the simulated events within the uncertainty of the distribution of the average number of interactions per bunch crossing. For the $\mathrm{Z}+$ jet region, this corresponds to a $\pm 4.6 \%$ shift in the total inelastic cross section [105], whereas for the dijet region it is a $+4.6 /-13.8 \%$ shift to account for larger variations coming from the use of prescaled triggers. Since the luminosity collected by prescaled triggers varies over the datataking periods, the pileup profile differs from the pileup distribution of the full dataset. Uncertainties related to the inelastic pp model [76] used to simulate pileup interactions are assumed to be negligible, because the variations in the model would produce a smaller impact on the charged particle multiplicity than the changes in the average number of interactions per bunch crossing (discussed above). No uncertainty related to the integrated luminosity is considered since this affects only the overall normalization, and therefore does not affect the normalized distributions presented in this paper. Uncertainties in the muon reconstruction and identification are negligible.

Uncertainties because of the choices of the factorization and renormalization scales $\left(\mu_{\mathrm{F}}, \mu_{\mathrm{R}}\right)$ are computed from the envelope of six variations by factors $(0.5,0.5),(0.5,1)$, $(1,0.5),(2,2),(2,1)$ and $(1,2)[106,107]$. The uncertainty from the PDFs is determined using the LO NNPDF replica set, where the root-mean-square of 100 pseudo-experiments provided by the PDF set represents the uncertainty. For the MG5+PYTHIA8 prediction, these uncertainties related to scale choices and PDFs mainly vary the prediction of jet momenta, whereas the substructure observables are only mildly modified. The uncertainty in the modelling of the parton shower and hadronization process is estimated using HER$\mathrm{WIG}++$ to construct an alternative response matrix. The statistical uncertainty in the HERWIG ++ simulation is small compared to the parton shower and hadronization uncertainty. This uncertainty is treated as single-sided when building the covariance matrix, since the experimental data distributions are generally enveloped by the MG5+PYTHIA 8 and HERWIG ++ predictions. We tested that rescaling the $p_{\mathrm{T}}$ spectrum of the HERWIG ++ prediction to match that of the MG5+PYTHIA8 prediction does not significantly change the estimated uncertainty, confirming that this uncertainty mainly captures differences in 

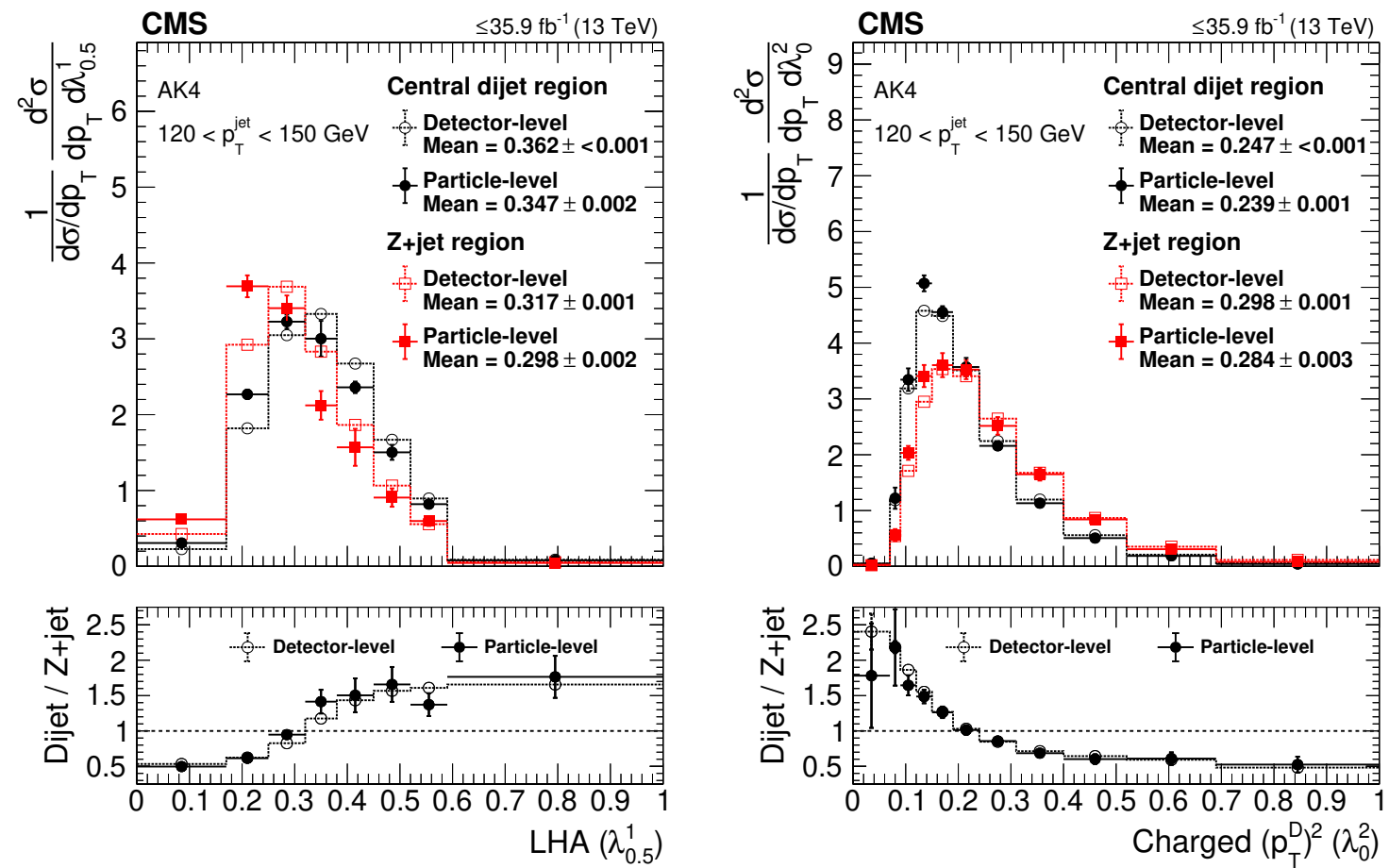

Figure 4. Detector-level and particle-level (unfolded) experimental data distributions of LHA $\left(\lambda_{0.5}^{1}\right)$ (left) using charged +neutral constituents, and $\left(p_{\mathrm{T}}^{\mathrm{D}}\right)^{2}\left(\lambda_{0}^{2}\right)$ (right) using only charged constituents, for jets with $120<p_{\mathrm{T}}<150 \mathrm{GeV}$ in the $\mathrm{Z}+$ jet (red) and central dijet (black) regions. The detectorlevel data uncertainties are statistical. The particle-level (unfolded) data uncertainties include the systematic components. Also shown is the mean of each distribution, calculated from the binned data. The ratio plots show the ratio of dijet to $\mathrm{Z}+$ jet distributions, for both the detector- and particle-level distributions.

the shower and hadronization modelling of substructure rather than the modelling of the $p_{\mathrm{T}}$ spectrum. We conclude that the mismodeling of the $p_{\mathrm{T}}$ spectrum in figure 3 has a negligible effect on the result.

The statistical uncertainty in the simulation that is used to derive the migration matrix is propagated through the unfolding and results in a contribution to the covariance matrix. The statistical uncertainties in the presented distributions correspond to those of the input experimental data, whereas statistical uncertainties in the response matrix are considered as part of the systematic uncertainties. The unfolding procedure reproduces the particle-level distributions when unfolding a statistically independent sample from the same generator that is used to derive the response matrix.

Figure 4 shows the distributions of two representative jet substructure observables in experimental data, computed using AK4 jets with $120<p_{\mathrm{T}}<150 \mathrm{GeV}$ in the central dijet and $\mathrm{Z}+$ jet regions, at both the detector and particle levels. The discrimination power between quark and gluon jets for each observable can be deduced by comparing the distributions in the $\mathrm{Z}+$ jet and central dijet regions that are enriched in quark and gluon jets, respectively. As previously mentioned, gluon jets exhibit typically larger values of LHA, width, thrust, and multiplicity, and smaller values of $\left(p_{\mathrm{T}}^{\mathrm{D}}\right)^{2}$, than quark jets. 
The distortion of the distribution by the detector effects can be inferred by comparing the detector-level data distributions with their corresponding particle-level (unfolded) data distributions. The charged $\left(p_{\mathrm{T}}^{\mathrm{D}}\right)^{2}$ (figure 4 , right) is an example where the difference between detector-level data and particle-level (unfolded) data is small as a result of considering only charged constituents. To aid comparison, the mean of each distribution is quoted. The mean is computed from the binned distribution, which is an approximation to the real mean of the underlying distribution, treating experimental data and simulation consistently, both at detector and particle levels. Although the uncertainty quoted in the mean of the detector-level data is purely statistical, the uncertainty quoted for the mean in particle-level (unfolded) data also includes systematic uncertainties.

Figure 5 shows the size of the considered uncertainties in the ungroomed LHA $\left(\lambda_{0.5}^{1}\right)$ for AK4 jets with $120<p_{\mathrm{T}}<150 \mathrm{GeV}$ in the central dijet region before (left) and after (right) the distribution for the given $p_{\mathrm{T}}$ bin is normalized. Although the JES uncertainty has the largest effect in the yield variations due to migration across the jet $p_{\mathrm{T}}$ boundaries, it plays a limited role for the normalized jet substructure distributions, where the shower and hadronization model uncertainty is typically dominant. The shape of the shower and hadronization uncertainty is related to some extend to the shape of the $\operatorname{LHA}\left(\lambda_{0.5}^{1}\right)$ distribution of HERWIG ++ , exhibiting a lower mean than MG5+PYTHIA8. The total systematic uncertainty is computed from the sum in quadrature of statistical and systematic uncertainties in each bin.

\section{Results and discussion}

Experimental data distributions unfolded to particle level in the $\mathrm{Z}+$ jet and central dijet regions are presented in figures 6-8 for the ungroomed substructure observables of AK4 jets with $120<p_{\mathrm{T}}<150 \mathrm{GeV}$. The data are compared with MG5+PYTHIA8 and HERWIG++ predictions at particle level. For the IRC-safe observables (LHA, width, and thrust) in the $\mathrm{Z}+$ jet region, the data are also compared with predictions from ref. [57] based on analytic resummation of large logarithms at next-to-leading logarithmic accuracy (NLL), matched to the exact NLO prediction, plus nonperturbative (NP) corrections derived from Sherpa, labeled NLO+NLL'+NP, where the superscript' of NLL indicates that a matching procedure keeping track of the jet flavour is used. The uncertainty band of the NLO+NLL'+NP prediction includes variations of factorization, renormalization and resummation scales as well as the model for NP corrections. For intermediate values of $\lambda_{\beta}^{\kappa}$ the prediction has small contributions from NP effects, which dominate at very low and high values of $\lambda_{\beta}^{\kappa}$.

As a simple quantitative assessment of agreement of each prediction with CMS data, values of $\chi^{2} / N_{\text {bins }}$ are quoted, where $N_{\text {bins }}$ is the number of bins of the distribution. The $\chi^{2} / N_{\text {bins }}$ is computed including the covariance between the measured cross sections including all statistical and experimental systematic uncertainties. For the NLO+NLL'+NP prediction theoretical uncertainties are taken into account as well, whereas for the MC generator prediction no theoretical systematic uncertainties are considered.

The agreement of the MG5+PYTHIA8 and HERWIG++ simulations with the experimental data varies depending on the observable and sample. In both $\mathrm{Z}+$ jet and central dijet regions, the MG5+PYTHIA8 and HERWIG ++ predictions tend to envelop the exper- 

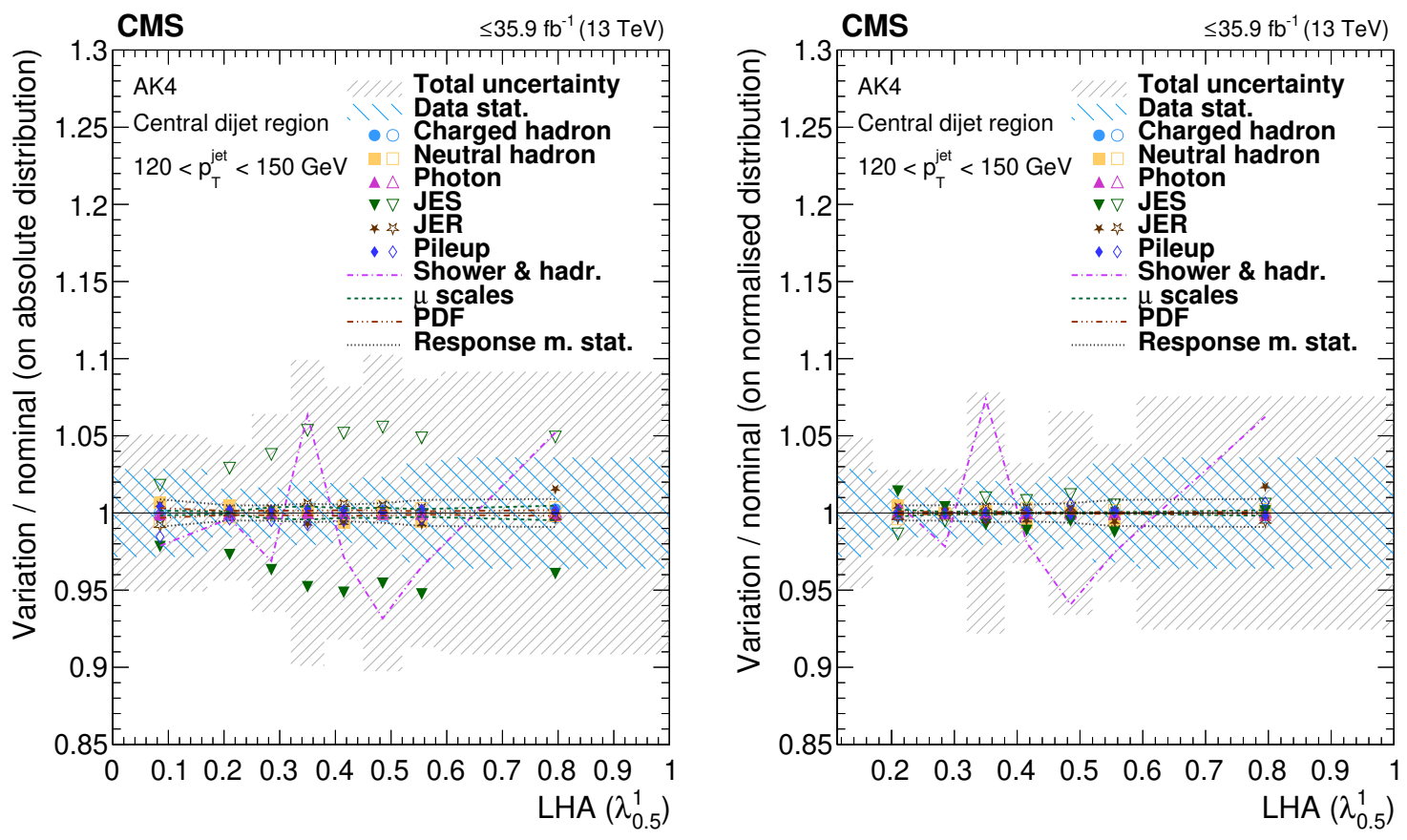

Figure 5. Ratio of the distribution that results from a varied response matrix to the nominal distribution of ungroomed LHA $\left(\lambda_{0.5}^{1}\right)$ for AK4 jets with $120<p_{\mathrm{T}}<150 \mathrm{GeV}$ in the central dijet region before (left) and after (right) normalization. Filled symbols correspond to upward variations, and the corresponding downward variations are represented by open symbols. The coarse-grained hashed region indicates the statistical uncertainty of the experimental data, and the fine-grained hashed region represents the total uncertainty, the sum in quadrature of statistical and systematic uncertainties.

imental data distributions. The significant difference between the MG5+PYTHIA8 and HERWIG++ predictions reflects the uncertainty in the prediction from MC event generators. These two predictions differ in the modelling of perturbative (matrix element, parton shower) and nonperturbative (hadronization) effects. In the $\mathrm{Z}+$ jet region, MG5+PYTHIA8 provides the best description of all angularities. HERWIG ++ predicts smaller values than MG5+PYTHIA8 for all observables, except $\left(p_{\mathrm{T}}^{\mathrm{D}}\right)^{2}$. In the central dijet region, the agreement of both generators is worse than in the $\mathrm{Z}+$ jet region. Here, HERWIG ++ shows a slightly better description of the experimental data than MG5+PYTHIA8, except for multiplicity and $\left(p_{\mathrm{T}}^{\mathrm{D}}\right)^{2}$.

The NLO+NLL'+NP prediction describes the thrust well, with a $\chi^{2} / N_{\text {bins }}$ below unity. The description of the width distribution is slightly worse, in particular at very low and high values of width, where NP effects contribute. The LHA shows a significant disagreement, indicating the need for further investigation of this prediction. These observations are consistent with the conclusions from ref. [57] where reasonable control of the thrust and width was obtained, although the LHA was not well under control. In the following, we focus on the LHA distribution where data may guide investigations. 

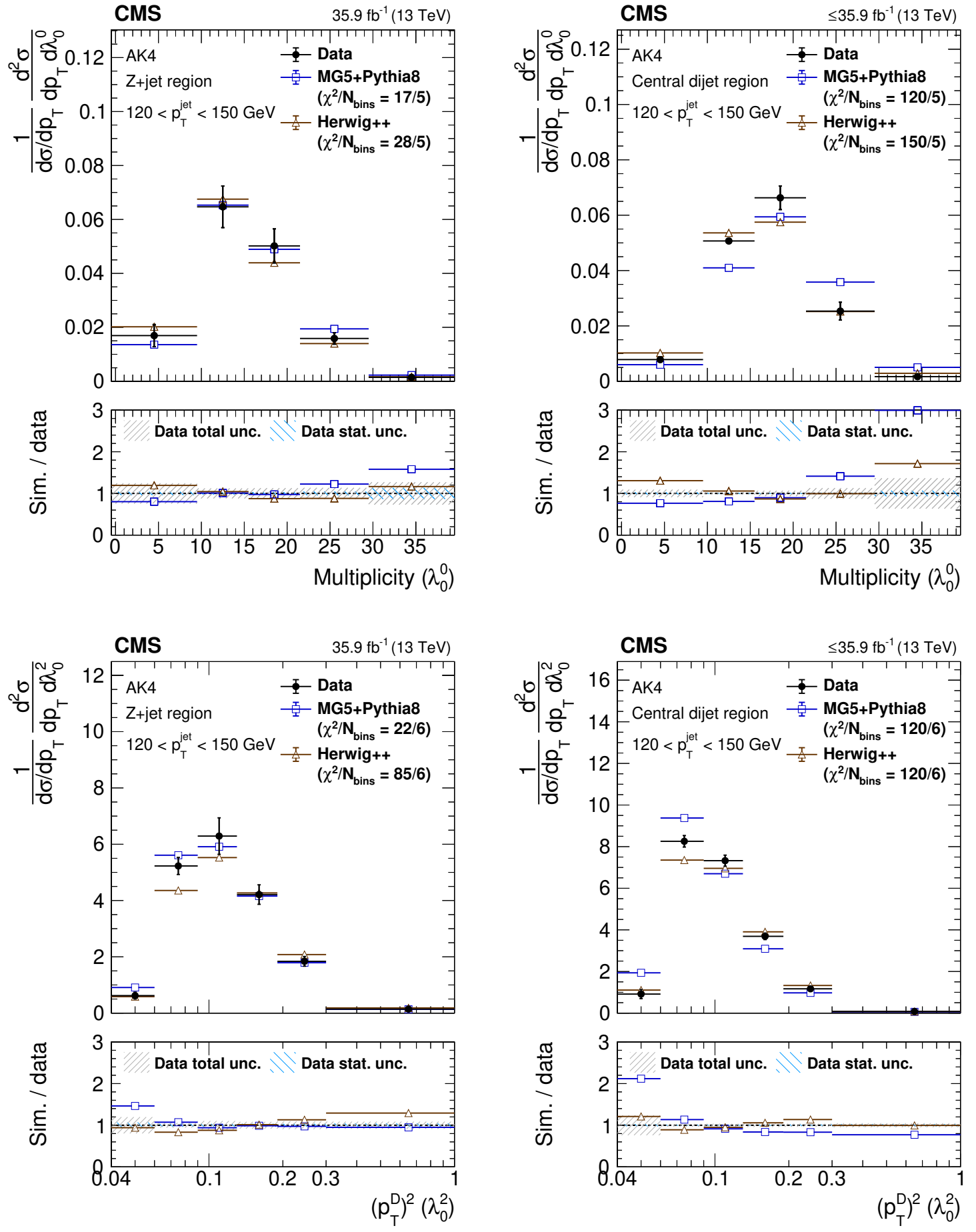

Figure 6. Particle-level distributions of (upper) ungroomed multiplicity $\left(\lambda_{0}^{0}\right)$ and (lower) ungroomed $\left(p_{\mathrm{T}}^{\mathrm{D}}\right)^{2}\left(\lambda_{0}^{2}\right)$ in $120<p_{\mathrm{T}}<150 \mathrm{GeV}$ in the $\mathrm{Z}+$ jet region (left) and central dijet region (right). The error bars on the data correspond to the total uncertainties. The coarse-grained blue hashed region in the ratio plot indicates the statistical uncertainty of the data, and the fine-grained grey hashed region represents the total uncertainty. The lowest bin extends down to $\lambda_{\beta}^{\kappa}>=0$. 

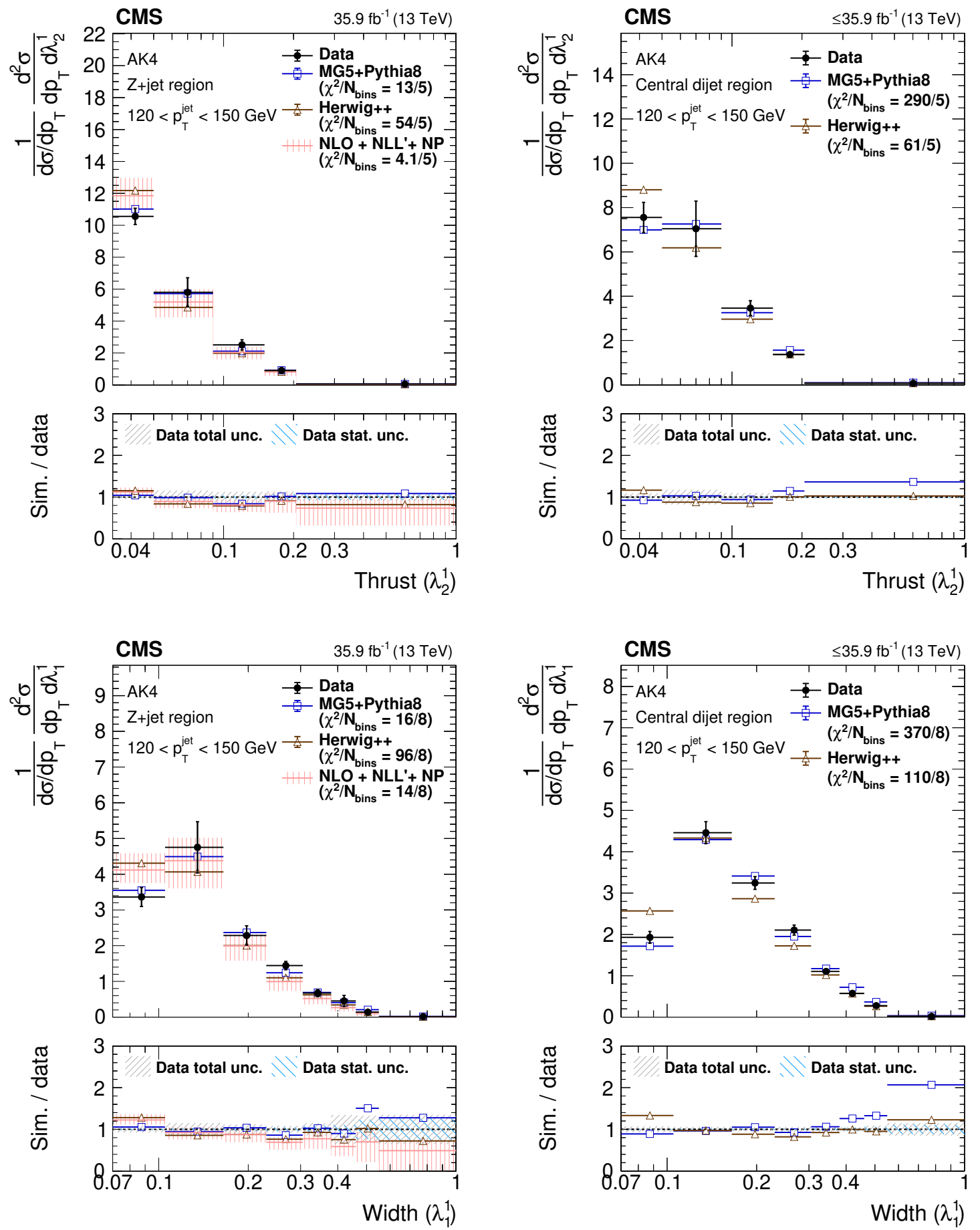

Figure 7. Particle-level distributions of (upper) ungroomed thrust $\left(\lambda_{2}^{1}\right)$ and (lower) ungroomed width $\left(\lambda_{1}^{1}\right)$ in $120<p_{\mathrm{T}}<150 \mathrm{GeV}$ in the $\mathrm{Z}+$ jet region (left) and central dijet region (right). The error bars on the data correspond to the total uncertainties. For the NLO+NLL'+NP prediction, the theory uncertainty is displayed as a red hashed band. The coarse-grained blue hashed region in the ratio plot indicates the statistical uncertainty of the data, and the fine-grained grey hashed region represents the total uncertainty. The lowest bin extends down to $\lambda_{\beta}^{\kappa}>=0$. 

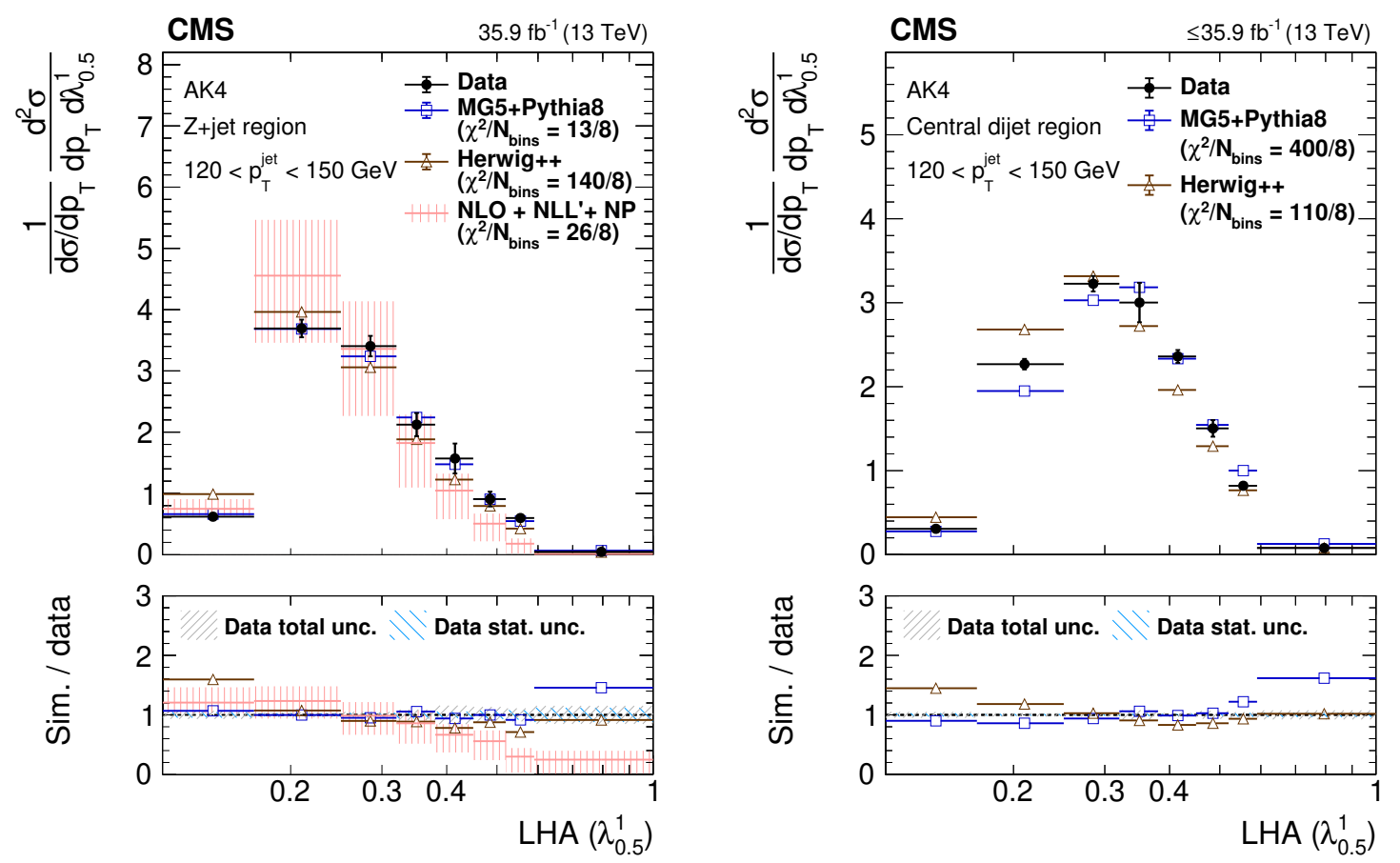

Figure 8. Particle-level distributions of ungroomed LHA $\left(\lambda_{0.5}^{1}\right)$ in $120<p_{\mathrm{T}}<150 \mathrm{GeV}$ in the $\mathrm{Z}+$ jet region (left) and central dijet region (right). The error bars on the data correspond to the total uncertainties. For the NLO+NLL' $+\mathrm{NP}$ prediction, the theory uncertainty is displayed as a red hashed band. The coarse-grained blue hashed region in the ratio plot indicates the statistical uncertainty of the data, and the fine-grained grey hashed region represents the total uncertainty. The lowest bin extends down to $\lambda_{\beta}^{\kappa}>=0$.

Figures 9-10 show multiple variants of LHA. Based on the $\chi^{2} / N_{\text {bins }}$, the level of datato-simulation agreement of MG5+PYTHIA8 for AK4 jets with $408<p_{\mathrm{T}}<1500 \mathrm{GeV}$ in the $\mathrm{Z}+$ jet region (figure 9, upper left) is worse than in the low- $p_{\mathrm{T}} \mathrm{Z}+$ jet region (figure 8 , left). The level of data-to-simulation agreement of MG5+PYTHIA 8 for $1<p_{\mathrm{T}}<4 \mathrm{TeV}$ in the central dijet region (enriched in quark jets, figure 9, upper right) is better than in the low- $p_{\mathrm{T}}$ central dijet region (enriched in gluon jets, figure 8, right). For HERWIG ++ , the level of agreement changes in opposite directions going from low- $p_{\mathrm{T}}$ to high- $p_{\mathrm{T}}$. This is consistent with the hypothesis that the level of agreement is related to the expected gluon fraction in the sample. The NLO+NLL'+NP prediction yields a similar level of agreement at low- $p_{\mathrm{T}}$ and high- $p_{\mathrm{T}}$.

The LHA distribution for AK8 jets with $120<p_{\mathrm{T}}<150 \mathrm{GeV}$ (figure 9 lower) is similar to that for AK4 jets. Its level of agreement between data and the MG5+PYTHIA 8 and $\mathrm{NLO}+\mathrm{NLL}+\mathrm{NP}$ predictions is worse than for AK4 jets, although the level of agreement remains more similar for HERWIG ++ .

Compared to LHA from charged+neutral constituents, the charged LHA distribution for ungroomed AK4 jets with $120<p_{\mathrm{T}}<150 \mathrm{GeV}$ (figure 10 upper) is binned more finely taking advantage of the good track resolution, resolving LHA values well below 0.1 . The 
data-to-theory agreement is similar in magnitude and shape above 0.1 , though the $\chi^{2} / N_{\text {bins }}$ is worse, suggesting that the majority of the underlying difference to experimental data in the theory prediction may be probed using a charged observable. This measurement is consistent with conclusions in ref. [57], where the NP corrections were very similar between charged+neutral and charged observables.

The LHA distribution for groomed AK4 jets with $120<p_{\mathrm{T}}<150 \mathrm{GeV}$ (figure 10 lower) is wider than for ungroomed jets. The data-to-theory agreement for LHA in groomed jets is similar compared with that in ungroomed jets. Although grooming is expected to reduce the influence from pileup, underlying event, and initial-state radiation, which are all difficult to model, we find no significant improvement in the description of LHA with grooming. This measurement is consistent with conclusions in ref. [57], where groomed LHA has a large remaining contribution from NP effects.

To study the behaviour of the measured jet substructure observables in the dimensions summarized in table 3, we focus on the mean value of each substructure distribution. Figure 11 shows the measured mean of ungroomed LHA $\left(\lambda_{0.5}^{1}\right)$ for AK4 jets as a function of jet $p_{\mathrm{T}}$, including also predictions from more recent generators. The mean decreases with the jet $p_{\mathrm{T}}$ in both the $\mathrm{Z}+$ jet and central dijet regions, as a result of constituents being located closer to the jet axis due to the larger Lorentz boost at higher $p_{\mathrm{T}}$. This trend is displayed by all generators. The mean in the $\mathrm{Z}+$ jet region is well-described at low $p_{\mathrm{T}}$ and overestimated at high $p_{\mathrm{T}}$ by MG5+PYTHIA8, whereas all other predictions significantly underestimate it across the entire $p_{\mathrm{T}}$ range. In the central dijet sample, MG5+PYTHIA 8 and HERWIG ++ generator predictions deviate significantly from the measurement. Although MG5+PYTHIA8 predicts a larger mean than that measured in the experimental data, HERWIG ++ underestimates it across the whole $p_{\mathrm{T}}$ range. The HERWIG7 $\mathrm{CH} 3$ and PYTHIA 8 CP2 simulations provide the best description, followed by PYTHIA8 CP5 and SHERPA. One can infer from this observation that the data-to-simulation agreement depends on how generators model the difference between quark and gluon jets, consistent with previous measurements of observables related to jet fragmentation [23].

Figure 12 summarizes the behaviour of jet substructure observables across all dimensions under study using the mean of each distribution. The upper plot in figure 12 shows the results from CMS data and the MG5+PYTHIA8 and HERWIG ++ simulations. Across most observables and dimensions, the measurements are enveloped by the two generator predictions. The behaviour as a function of $p_{\mathrm{T}}$ and jet radius parameter as well as the behaviour of charged and groomed observables discussed previously for LHA holds also for the other substructure observables.

Figure 12 (lower) compares the same measured data to predictions from more recent generators. In the gluon-enriched sample, HERWIG7 CH3, PYTHIA8 CP5, PYTHIA8 CP2, and SHERPA generally provide a better description than either HERWIG++ or MG5+PYTHIA8. In the quark-enriched sample, MG5+PYTHiA8 provides the best description, followed by HERWIG7, PYTHIA8 CP2, SHERPA, HERWIG++, and PYTHIA8 CP5. Thus, the previous observations in figure 12 (upper) of more accurate modelling of quark jet substructure, and less accurate modelling of gluon jet substructure, does not necessarily hold for more recent generators and tunes under study. Improved modelling of gluon jets at the cost of poorer 

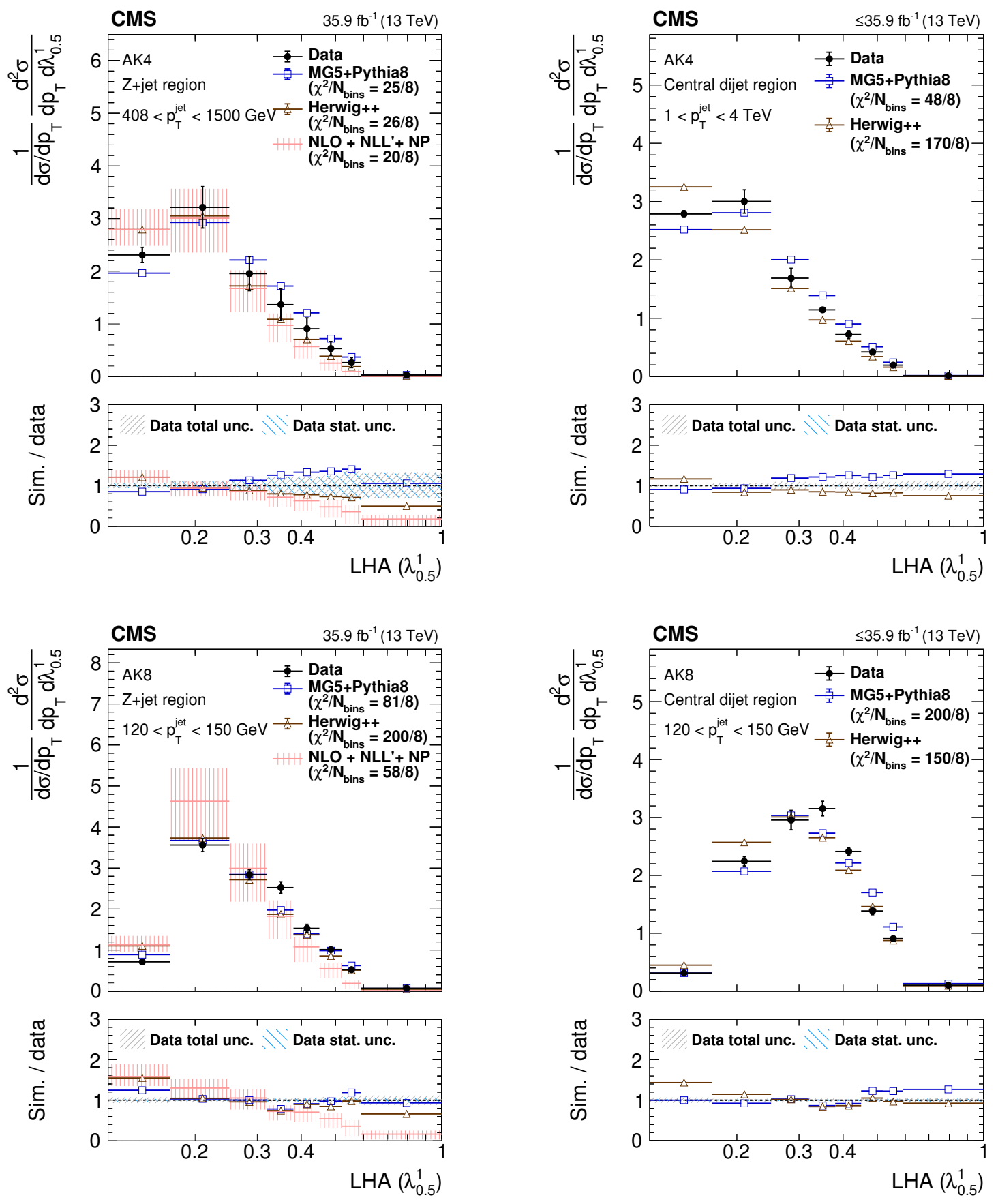

Figure 9. Particle-level distributions of (upper) ungroomed AK4 LHA $\left(\lambda_{0.5}^{1}\right)$ in $408<p_{\mathrm{T}}<$ $1500 \mathrm{GeV}$ in the $\mathrm{Z}+$ jet region (left) and in $1<p_{\mathrm{T}}<4 \mathrm{TeV}$ in the central dijet region (right) and (lower) ungroomed AK8 LHA $\left(\lambda_{0.5}^{1}\right)$ in AK8 $120<p_{\mathrm{T}}<150 \mathrm{GeV}$ in the $\mathrm{Z}+$ jet region (left) and central dijet region (right). The error bars on the data correspond to the total uncertainties. For the NLO+NLL'+NP prediction, the theory uncertainty is displayed as a red hashed band. The coarse-grained blue hashed region in the ratio plot indicates the statistical uncertainty of the data, and the fine-grained grey hashed region represents the total uncertainty. The lowest bin extends down to $\lambda_{\beta}^{\kappa}>=0$. 

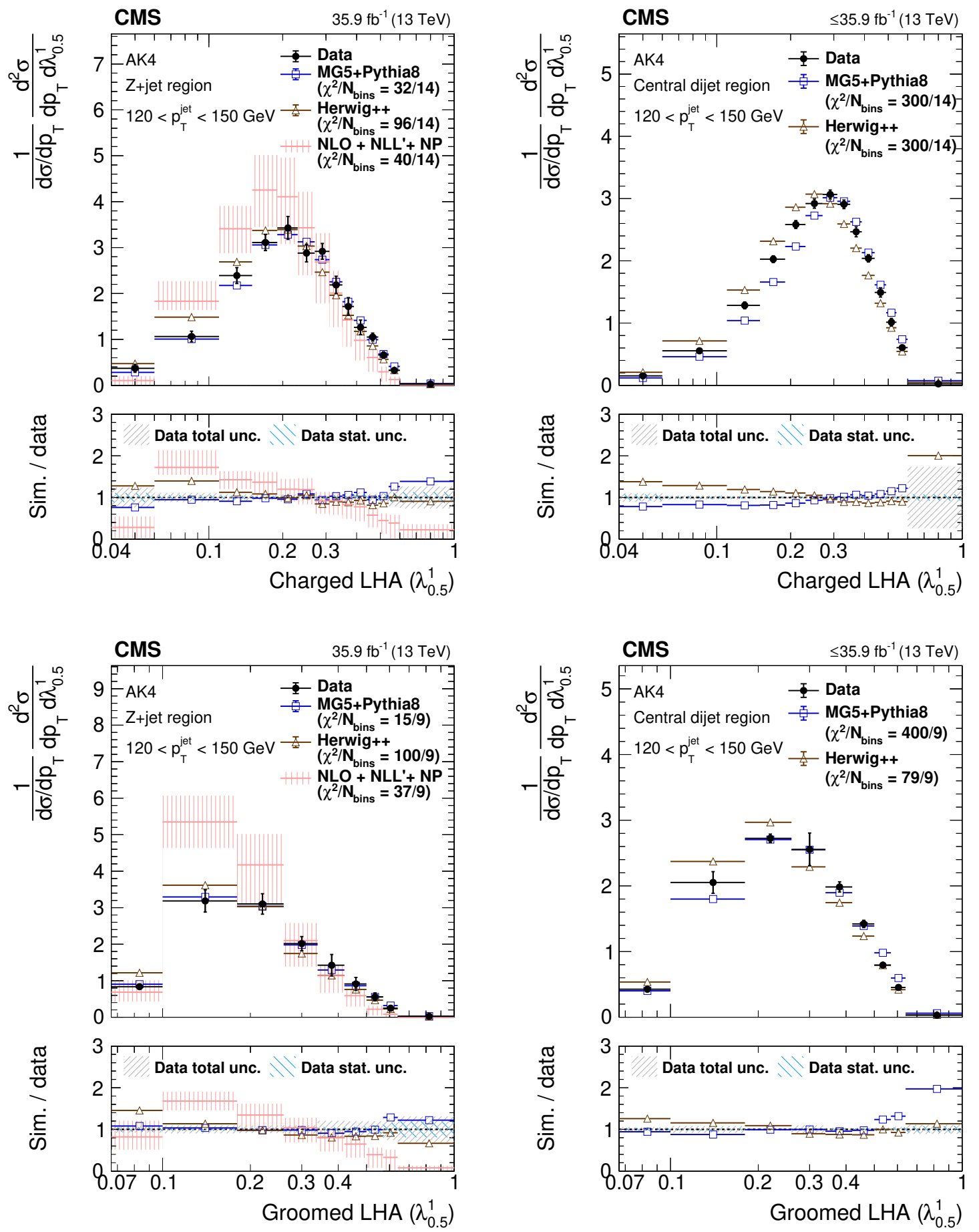

Figure 10. Particle-level distributions of (upper) ungroomed charged AK4 LHA $\left(\lambda_{0.5}^{1}\right)$ and (lower) groomed AK4 LHA $\left(\lambda_{0.5}^{1}\right)$ in $120<p_{\mathrm{T}}<150 \mathrm{GeV}$ in the $\mathrm{Z}+$ jet region (left) and central dijet region (right). The error bars on the data correspond to the total uncertainties. For the NLO+NLL'+NP prediction, the theory uncertainty is displayed as a red hashed band. The coarse-grained blue hashed region in the ratio plot indicates the statistical uncertainty of the data, and the fine-grained grey hashed region represents the total uncertainty. The lowest bin extends down to $\lambda_{\beta}^{\kappa}>=0$. 

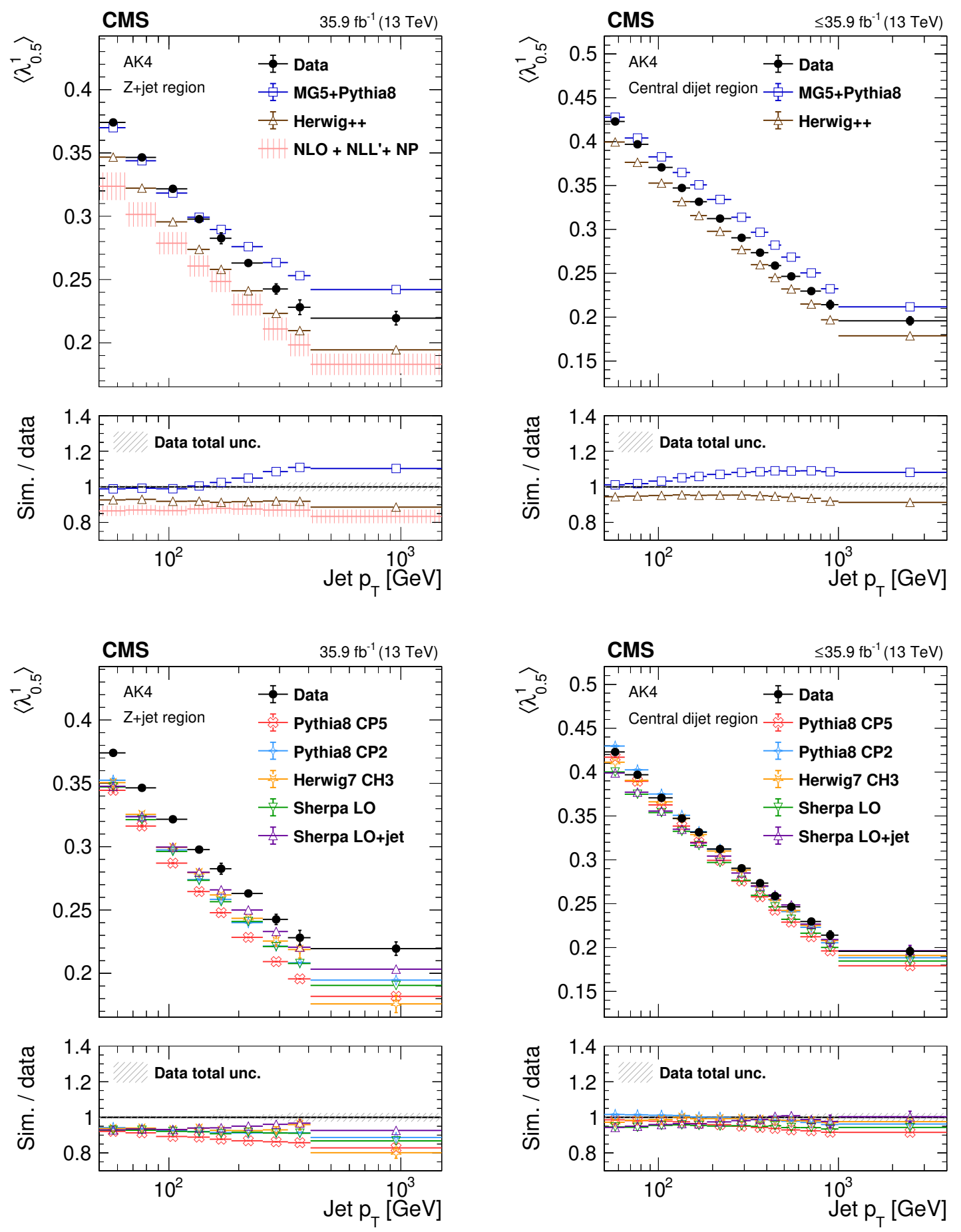

Figure 11. Mean value of ungroomed LHA $\left(\lambda_{0.5}^{1}\right)$ for AK4 jets as a function of $p_{\mathrm{T}}$ in the $\mathrm{Z}+$ jet (left) and central dijet region (right) regions. The upper and lower plots show the same data distribution compared with different generator predictions. The error bars on the data and the hashed region in the ratio plot correspond to the total uncertainties of the experimental data. The error bars on the simulation correspond to the statistical uncertainties. 

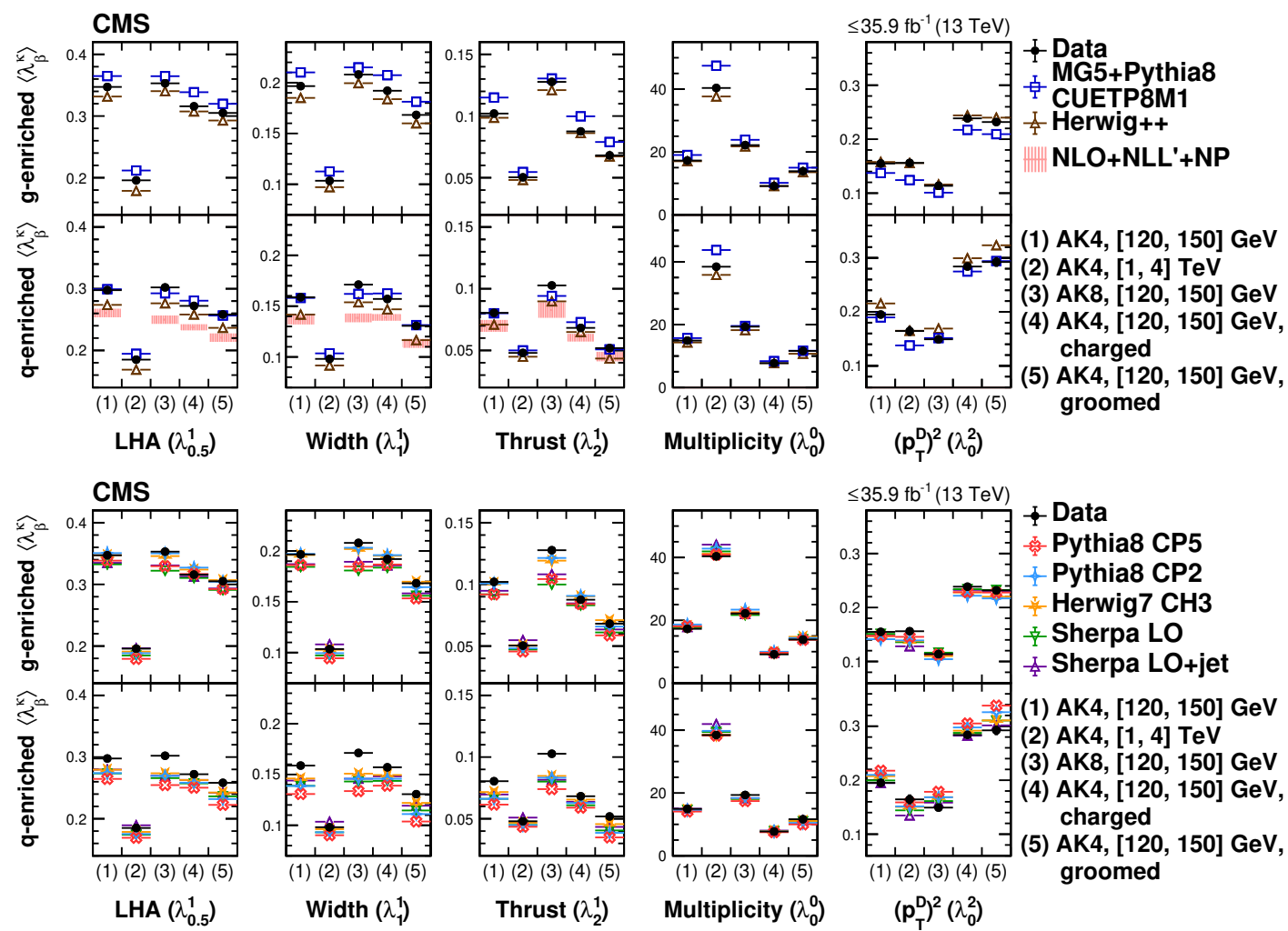

Figure 12. Mean value of substructure observables in regions with gluon-enriched and quarkenriched jets, for the following configurations: (1) ungroomed AK4 $120<p_{\mathrm{T}}<150 \mathrm{GeV}$, (2) ungroomed AK4 $1<p_{\mathrm{T}}<4 \mathrm{TeV}$, (3) ungroomed AK8 $120<p_{\mathrm{T}}<150 \mathrm{GeV}$, (4) ungroomed charged AK4 $120<p_{\mathrm{T}}<150 \mathrm{GeV}$, and (5) groomed AK4 $120<p_{\mathrm{T}}<150 \mathrm{GeV}$; shown for each of the observables LHA $\left(\lambda_{0.5}^{1}\right)$, width $\left(\lambda_{1}^{1}\right)$, thrust $\left(\lambda_{2}^{1}\right)$, multiplicity $\left(\lambda_{0}^{0}\right)$, and $\left(p_{\mathrm{T}}^{\mathrm{D}}\right)^{2}\left(\lambda_{0}^{2}\right)$. The central jet in the dijet region is used for the gluon-enriched sample, whereas for the quark-enriched sample the jet in the $\mathrm{Z}+$ jet region is used for $120<p_{\mathrm{T}}<150 \mathrm{GeV}$, and the forward jet in the dijet region is used for $1<p_{\mathrm{T}}<4 \mathrm{TeV}$. The upper and lower plots show the same data distribution compared with different generator predictions. The error bars on the data correspond to the total uncertainties. The error bars on the simulation correspond to the statistical uncertainties.

modelling of quark jets is observed. In addition to the more recent generator versions and tunes used for HERWIG and PYTHIA8, an important difference among the predictions is the choice of $\alpha_{\mathrm{S}}\left(m_{\mathrm{Z}}\right)$ in the final state shower, to which the jet substructure modelling is sensitive [41]. The PYTHIA8 CP2 prediction using $\alpha_{\mathrm{S}}\left(m_{\mathrm{Z}}\right)=0.130$ predicts systematically larger values of LHA, width, thrust, and multiplicity, and smaller value of $\left(p_{\mathrm{T}}^{\mathrm{D}}\right)^{2}$, for both quarks and gluons than the PYTHIA8 CP5 prediction using $\alpha_{\mathrm{S}}\left(m_{\mathrm{Z}}\right)=0.118$.

Figure 13 shows the ratio of the means in the gluon- and quark-enriched samples, illustrating how well the generators model both the differences between quarks and gluons, and the relative quark/gluon composition of the samples. As previously mentioned, gluon jets exhibit, on average, larger values of LHA, width, thrust, and multiplicity, and smaller values of $\left(p_{\mathrm{T}}^{\mathrm{D}}\right)^{2}$, than quark jets. At low $p_{\mathrm{T}}$, the ratio of the means in the central dijet and $\mathrm{Z}+$ jet regions is significantly larger than unity for LHA, width, thrust, and multiplicity, and 
significantly smaller for $\left(p_{\mathrm{T}}^{\mathrm{D}}\right)^{2}$, indicating that these observables have significant separation power between quark and gluon jets. Strikingly, all generators overestimate the difference between quark and gluon jets at low $p_{\mathrm{T}}$ when compared with experimental data. At high $p_{\mathrm{T}}$, however, the ratio of the means in the central and forward dijet regions is significantly closer to unity, and all generators give a reasonable description of the ratio that is consistent with that measured in data. The gluon- and quark-enriched samples are each expected to have a more equal relative quark/gluon jet composition at this $p_{\mathrm{T}}$ (see figure 2). The best overall data-to-simulation agreement for the ratio is achieved by SHERPA, followed by HERWIG ++ , MG5+PYTHIA8, HeRWig7 CH3, PYTHIA8 CP2, and PYTHIA8 CP5. The PYTHia8 CP2 and CP5 predictions are very similar, showing that the quark and gluon discrimination power is not governed by the choice of $\alpha_{\mathrm{S}}\left(m_{\mathrm{Z}}\right)$ in the final state shower, but rather by the model used for jet fragmentation. Comparing the behaviour of SHERPA LO to LO+jet, the effect of the additional outgoing partons is an improvement in the description of the ratio of the means in the gluon- and quark-enriched samples. Since the additional outgoing partons can affect the fraction of gluon jets in the signal regions, this demonstrates that the measurement of the ratio provides information not only about the differences between quarks and gluons, but also about the fraction of gluon jets. A higher order prediction of the angularities in the dijet region, matching the precision of the NLO+NLL'+NP predictions in the $\mathrm{Z}+$ jet region, may yield better understanding of the source of this disagreement.

\section{Summary}

Measurements of distributions of generalized jet angularities in proton-proton collision data taken by the CMS detector at $\sqrt{s}=13 \mathrm{TeV}$ in dijet and, for the first time, also in Z+jet topologies have been presented. Whereas the dijet topology allows access to a sample of jets that predominantly originate from gluon fragmentation, the $\mathrm{Z}+$ jet topology yields a sample enriched in quark-initiated jets. Five generalized angularities are measured to study different features in the modelling of jet substructure. Three infrared- and collinear-safe angularities are particularly sensitive to perturbative emissions in jets, whereas the other two have larger contributions from nonperturbative effects. For the first time, a measurement of angularities with different jet radii, both with and without the application of a grooming algorithm, was carried out to further discriminate between different features in the modelling. Although a subset of these distributions was discussed in this paper, the full range of measurements is made public in HEPData record [102]. The measurements for quark and gluon jets yield values in between the predictions from the MADGRAPH5_aMC@NLO+PYTHIA8 and HERWIG++ simulations. The quality of modelling for the infrared- and collinear-safe angularities is sensitive to the quark and gluon composition of the sample of jets. Although grooming is expected to reduce the influence from pileup, underlying event, and initial-state radiation, which are all difficult to model, we find no significant improvement in the description with grooming. A calculation based on analytic resummation of large logarithms of the collinear-safe angularities in the $\mathrm{Z}+$ jet topology at next-to-leading order + next-to-leading logarithm (NLO+NLL') accuracy including nonperturbative effects best describes the thrust with a $\chi^{2} / N_{\text {bins }}$ (where $N_{\text {bins }}$ is the number 

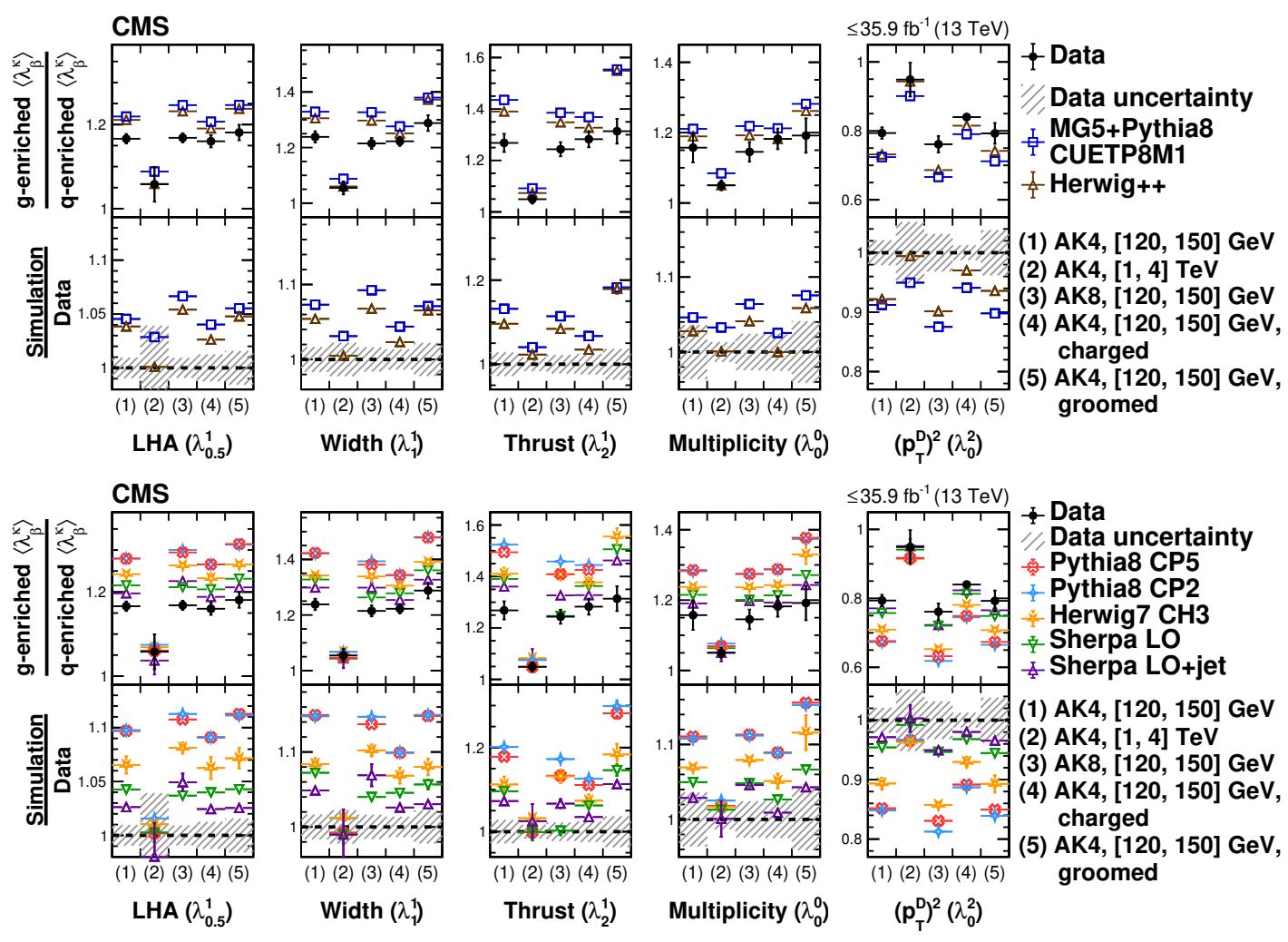

Figure 13. Ratio of the mean of substructure observables in regions with gluon- and quark-enriched jets, for the following configurations: (1) ungroomed AK4 $120<p_{\mathrm{T}}<150 \mathrm{GeV}$, (2) ungroomed AK4 $1<p_{\mathrm{T}}<4 \mathrm{TeV}$, (3) ungroomed AK8 $120<p_{\mathrm{T}}<150 \mathrm{GeV}$, (4) ungroomed charged AK4 $120<p_{\mathrm{T}}<150 \mathrm{GeV}$, and (5) groomed AK4 $120<p_{\mathrm{T}}<150 \mathrm{GeV}$; for the observables LHA $\left(\lambda_{0.5}^{1}\right)$, width $\left(\lambda_{1}^{1}\right)$, thrust $\left(\lambda_{2}^{1}\right)$, multiplicity $\left(\lambda_{0}^{0}\right)$, and $\left(p_{\mathrm{T}}^{\mathrm{D}}\right)^{2}\left(\lambda_{0}^{2}\right)$. The central jet in the dijet region is used for the gluon-enriched sample, whereas for the quark-enriched sample the jet in the $\mathrm{Z}+$ jet region is used for $120<p_{\mathrm{T}}<150 \mathrm{GeV}$, whereas the forward jet in the dijet region is used for $1<p_{\mathrm{T}}<4 \mathrm{TeV}$. The upper and lower plots show the same data distribution compared with different generator predictions. The error bars on the data correspond to the total uncertainties. The error bars on the simulation correspond to the statistical uncertainties.

of bins of the distribution) as low as 4.1/5.0, whereas the Les Houches Angularity was described significantly worse with $\chi^{2} / N_{\text {bins }}$ up to 58/8. A comparison of the means of the angularities in quark- and gluon-enriched data samples demonstrated their discrimination power, which was overestimated by all generators under study, showing a clear need for improvements in the simulation.

\section{Acknowledgments}

We congratulate our colleagues in the CERN accelerator departments for the excellent performance of the LHC and thank the technical and administrative staffs at CERN and at other CMS institutes for their contributions to the success of the CMS effort. In addition, we gratefully acknowledge the computing centres and personnel of the Worldwide LHC 
Computing Grid and other centres for delivering so effectively the computing infrastructure essential to our analyses. Finally, we acknowledge the enduring support for the construction and operation of the LHC, the CMS detector, and the supporting computing infrastructure provided by the following funding agencies: BMBWF and FWF (Austria); FNRS and FWO (Belgium); CNPq, CAPES, FAPERJ, FAPERGS, and FAPESP (Brazil); MES and BNSF (Bulgaria); CERN; CAS, MoST, and NSFC (China); MINCIENCIAS (Colombia); MSES and CSF (Croatia); RIF (Cyprus); SENESCYT (Ecuador); MoER, ERC PUT and ERDF (Estonia); Academy of Finland, MEC, and HIP (Finland); CEA and CNRS/IN2P3 (France); BMBF, DFG, and HGF (Germany); GSRI (Greece); NKFIA (Hungary); DAE and DST (India); IPM (Iran); SFI (Ireland); INFN (Italy); MSIP and NRF (Republic of Korea); MES (Latvia); LAS (Lithuania); MOE and UM (Malaysia); BUAP, CINVESTAV, CONACYT, LNS, SEP, and UASLP-FAI (Mexico); MOS (Montenegro); MBIE (New Zealand); PAEC (Pakistan); MSHE and NSC (Poland); FCT (Portugal); JINR (Dubna); MON, RosAtom, RAS, RFBR, and NRC KI (Russia); MESTD (Serbia); SEIDI, CPAN, PCTI, and FEDER (Spain); MOSTR (Sri Lanka); Swiss Funding Agencies (Switzerland); MST (Taipei); ThEPCenter, IPST, STAR, and NSTDA (Thailand); TUBITAK and TAEK (Turkey); NASU (Ukraine); STFC (United Kingdom); DOE and NSF (U.S.A.).

Individuals have received support from the Marie-Curie programme and the European Research Council and Horizon 2020 Grant, contract Nos. 675440, 724704, 752730, 758316, 765710, 824093, 884104, and COST Action CA16108 (European Union); the Leventis Foundation; the Alfred P. Sloan Foundation; the Alexander von Humboldt Foundation; the Belgian Federal Science Policy Office; the Fonds pour la Formation à la Recherche dans l'Industrie et dans l'Agriculture (FRIA-Belgium); the Agentschap voor Innovatie door Wetenschap en Technologie (IWT-Belgium); the F.R.S.-FNRS and FWO (Belgium) under the "Excellence of Science - EOS" — be.h project n. 30820817; the Beijing Municipal Science \& Technology Commission, No. Z191100007219010; the Ministry of Education, Youth and Sports (MEYS) of the Czech Republic; the Deutsche Forschungsgemeinschaft (DFG), under Germany's Excellence Strategy - EXC 2121 "Quantum Universe" - 390833306, and under project number 400140256 - GRK2497; the Lendület ("Momentum") Programme and the János Bolyai Research Scholarship of the Hungarian Academy of Sciences, the New National Excellence Program ÚNKP, the NKFIA research grants 123842, 123959, 124845, 124850, 125105, 128713, 128786, and 129058 (Hungary); the Council of Science and Industrial Research, India; the Latvian Council of Science; the Ministry of Science and Higher Education and the National Science Center, contracts Opus 2014/15/B/ST2/03998 and 2015/19/B/ST2/02861 (Poland); the Fundação para a Ciência e a Tecnologia, grant CEECIND/01334/2018 (Portugal); the National Priorities Research Program by Qatar National Research Fund; the Ministry of Science and Higher Education, project no. 14.W03.31.0026 (Russia); the Programa Estatal de Fomento de la Investigación Científica y Técnica de Excelencia María de Maeztu, grant MDM-2015-0509 and the Programa Severo Ochoa del Principado de Asturias; the Stavros Niarchos Foundation (Greece); the Rachadapisek Sompot Fund for Postdoctoral Fellowship, Chulalongkorn University and the Chulalongkorn Academic into Its 2nd Century Project Advancement Project (Thailand); the Kavli Foundation; the Nvidia Corporation; the SuperMicro Corporation; the Welch Foundation, contract C-1845; and the Weston Havens Foundation (U.S.A.). 
Open Access. This article is distributed under the terms of the Creative Commons Attribution License (CC-BY 4.0), which permits any use, distribution and reproduction in any medium, provided the original author(s) and source are credited.

\section{References}

[1] G.P. Salam, Towards jetography, Eur. Phys. J. C 67 (2010) 637 [arXiv:0906.1833] [INSPIRE].

[2] R. Kogler et al., Jet substructure at the Large Hadron Collider, Rev. Mod. Phys. 91 (2019) 045003 [arXiv: 1803.06991] [INSPIRE].

[3] A.J. Larkoski, I. Moult and B. Nachman, Jet substructure at the Large Hadron Collider: a review of recent advances in theory and machine learning, Phys. Rept. 841 (2020) 1 [arXiv: 1709.04464] [INSPIRE].

[4] S. Marzani, G. Soyez and M. Spannowsky, Looking inside jets: an introduction to jet substructure and boosted-object phenomenology, Lect. Notes Phys. 958 (2019) 1 [arXiv: 1901.10342] [INSPIRE].

[5] P. Gras et al., Systematics of quark/gluon tagging, JHEP 07 (2017) 091 [arXiv: 1704.03878] [INSPIRE].

[6] K. Konishi, A. Ukawa and G. Veneziano, Jet calculus: a simple algorithm for resolving QCD jets, Nucl. Phys. B 157 (1979) 45 [InSPIRE].

[7] OPAL collaboration, A study of differences between quark and gluon jets using vertex tagging of quark jets, Z. Phys. C 58 (1993) 387 [INSPIRE].

[8] OPAL collaboration, A model independent measurement of quark and gluon jet properties and differences, Z. Phys. C 68 (1995) 179 [inSPIRE].

[9] DELPHI collaboration, Energy dependence of the differences between the quark and gluon jet fragmentation, Z. Phys. C $\mathbf{7 0}$ (1996) 179 [INSPIRE].

[10] ALEPH collaboration, Measurements of the structure of quark and gluon jets in hadronic $Z$ decays, Eur. Phys. J. C 17 (2000) 1 [INSPIRE].

[11] ALEPH collaboration, Quark and gluon jet properties in symmetric three jet events, Phys. Lett. B 384 (1996) 353 [INSPIRE].

[12] ALEPH collaboration, Studies of quantum chromodynamics with the ALEPH detector, Phys. Rept. 294 (1998) 1 [inSPIRE].

[13] ATLAS collaboration, Properties of jet fragmentation using charged particles measured with the ATLAS detector in pp collisions at $\sqrt{s}=13 \mathrm{TeV}$, Phys. Rev. D 100 (2019) 052011 [arXiv: 1906.09254] [INSPIRE].

[14] ATLAS collaboration, Properties of $g \rightarrow b \bar{b}$ at small opening angles in pp collisions with the ATLAS detector at $\sqrt{s}=13 \mathrm{TeV}$, Phys. Rev. D 99 (2019) 052004 [arXiv:1812.09283] [INSPIRE].

[15] ATLAS collaboration, Measurement of jet fragmentation in $5.02 \mathrm{TeV}$ proton-lead and proton-proton collisions with the ATLAS detector, Nucl. Phys. A 978 (2018) 65 [arXiv: 1706. 02859] [INSPIRE]. 
[16] ATLAS collaboration, Measurement of jet fragmentation in $\mathrm{Pb}+\mathrm{Pb}$ and $\mathrm{pp}$ collisions at $\sqrt{s_{N N}}=2.76 \mathrm{TeV}$ with the ATLAS detector at the LHC, Eur. Phys. J. C 77 (2017) 379 [arXiv: 1702.00674] [INSPIRE].

[17] ATLAS collaboration, Measurement of the charged-particle multiplicity inside jets from $\sqrt{s}=8 \mathrm{TeV}$ pp collisions with the ATLAS detector, Eur. Phys. J. C 76 (2016) 322 [arXiv: 1602.00988] [INSPIRE].

[18] ATLAS collaboration, Measurement of the jet fragmentation function and transverse profile in proton-proton collisions at a center-of-mass energy of $7 \mathrm{TeV}$ with the ATLAS detector, Eur. Phys. J. C 71 (2011) 1795 [arXiv:1109.5816] [inSPIRE].

[19] ATLAS collaboration, Properties of jets measured from tracks in proton-proton collisions at center-of-mass energy $\sqrt{s}=7 \mathrm{TeV}$ with the ATLAS detector, Phys. Rev. D 84 (2011) 054001 [arXiv: 1107.3311] [INSPIRE].

[20] CMS collaboration, Jet shapes of isolated photon-tagged jets in $\mathrm{Pb}-\mathrm{Pb}$ and $\mathrm{pp}$ collisions at $\sqrt{s_{N N}}=5.02 \mathrm{TeV}$, Phys. Rev. Lett. 122 (2019) 152001 [arXiv:1809.08602] [INSPIRE].

[21] CMS collaboration, Measurement of the splitting function in pp and Pb-Pb collisions at $\sqrt{s_{N N}}=5.02 \mathrm{TeV}$, Phys. Rev. Lett. 120 (2018) 142302 [arXiv:1708.09429] [INSPIRE].

[22] CMS collaboration, Measurement of jet fragmentation in $\mathrm{Pb}-\mathrm{Pb}$ and $\mathrm{pp}$ collisions at $\sqrt{s_{N N}}=2.76 \mathrm{TeV}$, Phys. Rev. C 90 (2014) 024908 [arXiv:1406.0932] [InSPIRE].

[23] CMS collaboration, Shape, transverse size, and charged hadron multiplicity of jets in pp collisions at $7 \mathrm{TeV}$, JHEP 06 (2012) 160 [arXiv:1204.3170] [INSPIRE].

[24] CMS collaboration, Measurement of jet fragmentation into charged particles in pp and $\mathrm{PbPb}$ collisions at $\sqrt{s_{N N}}=2.76 \mathrm{TeV}$, JHEP 10 (2012) 087 [arXiv: 1205.5872] [INSPIRE].

[25] ALICE collaboration, Exploration of jet substructure using iterative declustering in pp and Pb-Pb collisions at LHC energies, Phys. Lett. B 802 (2020) 135227 [arXiv: 1905.02512] [INSPIRE].

[26] ALICE collaboration, Measurement of the production of charm jets tagged with $D^{0}$ mesons in pp collisions at $\sqrt{s}=7 \mathrm{TeV}$, JHEP 08 (2019) 133 [arXiv:1905.02510] [INSPIRE].

[27] ALICE collaboration, Jet fragmentation transverse momentum measurements from di-hadron correlations in $\sqrt{s}=7 \mathrm{TeV} p p$ and $\sqrt{s_{N N}}=5.02 \mathrm{TeV} p$-Pb collisions, JHEP 03 (2019) 169 [arXiv: 1811.09742] [InSPIRE].

[28] ALICE collaboration, Charged jet cross section and fragmentation in proton-proton collisions at $\sqrt{s}=7 \mathrm{TeV}$, Phys. Rev. D 99 (2019) 012016 [arXiv:1809.03232] [inSPIRE].

[29] LHCb collaboration, Measurement of charged hadron production in Z-tagged jets in proton-proton collisions at $\sqrt{s}=8 \mathrm{TeV}$, Phys. Rev. Lett. 123 (2019) 232001 [arXiv: 1904.08878] [INSPIRE].

[30] ATLAS collaboration, Measurement of the Lund jet plane using charged particles in $13 \mathrm{TeV}$ proton-proton collisions with the ATLAS detector, Phys. Rev. Lett. 124 (2020) 222002 [arXiv: 2004.03540] [INSPIRE].

[31] ATLAS collaboration, Measurement of soft-drop jet observables in pp collisions with the ATLAS detector at $\sqrt{s}=13 \mathrm{TeV}$, Phys. Rev. D 101 (2020) 052007 [arXiv:1912.09837] [INSPIRE].

[32] ATLAS collaboration, Measurement of jet-substructure observables in top quark, $W$ boson and light jet production in proton-proton collisions at $\sqrt{s}=13 \mathrm{TeV}$ with the ATLAS detector, JHEP 08 (2019) 033 [arXiv:1903.02942] [INSPIRE]. 
[33] ATLAS collaboration, Measurement of the jet mass in high transverse momentum $Z(\rightarrow b \bar{b}) \gamma$ production at $\sqrt{s}=13 \mathrm{TeV}$ using the ATLAS detector, Phys. Lett. B 812 (2021) 135991 [arXiv: 1907.07093] [INSPIRE].

[34] ATLAS collaboration, Measurement of colour flow using jet-pull observables in t $\bar{t}$ events with the ATLAS experiment at $\sqrt{s}=13 \mathrm{TeV}$, Eur. Phys. J. C 78 (2018) 847 [arXiv: 1805.02935] [INSPIRE].

[35] ATLAS collaboration, Measurement of the soft-drop jet mass in pp collisions at $\sqrt{s}=13 \mathrm{TeV}$ with the ATLAS detector, Phys. Rev. Lett. 121 (2018) 092001 [arXiv: 1711.08341] [INSPIRE].

[36] ATLAS collaboration, Measurement of colour flow with the jet pull angle in $t \bar{t}$ events using the ATLAS detector at $\sqrt{s}=8 \mathrm{TeV}$, Phys. Lett. B 750 (2015) 475 [arXiv:1506.05629] [INSPIRE].

[37] ATLAS collaboration, Measurement of jet charge in dijet events from $\sqrt{s}=8 \mathrm{TeV} p p$ collisions with the ATLAS detector, Phys. Rev. D 93 (2016) 052003 [arXiv:1509.05190] [INSPIRE].

[38] ATLAS collaboration, Measurement of jet shapes in top-quark pair events at $\sqrt{s}=7 \mathrm{TeV}$ using the ATLAS detector, Eur. Phys. J. C 73 (2013) 2676 [arXiv:1307.5749] [InSPIRE].

[39] ATLAS collaboration, ATLAS measurements of the properties of jets for boosted particle searches, Phys. Rev. D 86 (2012) 072006 [arXiv:1206.5369] [InSPIRE].

[40] ATLAS collaboration, Jet mass and substructure of inclusive jets in $\sqrt{s}=7 \mathrm{TeV} p p$ collisions with the ATLAS experiment, JHEP 05 (2012) 128 [arXiv: 1203.4606] [INSPIRE].

[41] CMS collaboration, Measurement of jet substructure observables in t $\bar{t}$ events from proton-proton collisions at $\sqrt{s}=13 \mathrm{TeV}$, Phys. Rev. D 98 (2018) 092014 [arXiv: 1808.07340] [INSPIRE].

[42] CMS collaboration, Measurements of the differential jet cross section as a function of the jet mass in dijet events from proton-proton collisions at $\sqrt{s}=13 \mathrm{TeV}$, JHEP 11 (2018) 113 [arXiv: 1807.05974] [INSPIRE].

[43] CMS collaboration, Measurement of the jet mass in highly boosted t $\bar{t}$ events from $p p$ collisions at $\sqrt{s}=8 \mathrm{TeV}$, Eur. Phys. J. C 77 (2017) 467 [arXiv:1703.06330] [INSPIRE].

[44] CMS collaboration, Measurements of jet charge with dijet events in pp collisions at $\sqrt{s}=8 \mathrm{TeV}$, JHEP 10 (2017) 131 [arXiv:1706.05868] [INSPIRE].

[45] CMS collaboration, Studies of jet mass in dijet and $W / Z+$ jet events, JHEP 05 (2013) 090 [arXiv: 1303.4811] [INSPIRE].

[46] ALICE collaboration, Measurement of the groomed jet radius and momentum splitting fraction in pp and $\mathrm{Pb}-\mathrm{Pb}$ collisions at $\sqrt{s_{N N}}=5.02 \mathrm{TeV}$, submitted to Phys. Rev. Lett. [arXiv:2107.12984] [INSPIRE].

[47] ALICE collaboration, Measurements of the groomed and ungroomed jet angularities in pp collisions at $\sqrt{s}=5.02 \mathrm{TeV}$, arXiv:2107.11303 [INSPIRE].

[48] A.J. Larkoski, J. Thaler and W.J. Waalewijn, Gaining (mutual) information about quark/gluon discrimination, JHEP 11 (2014) 129 [arXiv:1408.3122] [INSPIRE].

[49] J.R. Andersen et al., Les Houches 2015: physics at TeV colliders Standard Model working group report, in $9^{\text {th }}$ Les Houches workshop on physics at TeV colliders, (2016) [arXiv: 1605. 04692] [INSPIRE]. 
[50] E. Farhi, Quantum chromodynamics test for jets, Phys. Rev. Lett. 39 (1977) 1587 [INSPIRE].

[51] CMS collaboration, Performance of quark/gluon discrimination in $8 \mathrm{TeV}$ pp data, CMS Physics Analysis Summary CMS-PAS-JME-13-002, CERN, Geneva, Switzerland (2013).

[52] CMS collaboration, Jet algorithms performance in $13 \mathrm{TeV}$ data, CMS Physics Analysis Summary CMS-PAS-JME-16-003, CERN, Geneva, Switzerland (2017).

[53] ATLAS collaboration, Light-quark and gluon jet discrimination in pp collisions at $\sqrt{s}=7 \mathrm{TeV}$ with the ATLAS detector, Eur. Phys. J. C 74 (2014) 3023 [arXiv:1405.6583] [INSPIRE].

[54] S. Caletti, O. Fedkevych, S. Marzani and D. Reichelt, Tagging the initial-state gluon, Eur. Phys. J. C 81 (2021) 844 [arXiv:2108.10024] [inSPIRE].

[55] R. Kumar, M. Bansal and S. Bansal, Jet fragmentation as a tool to explore double parton scattering using Z-boson + jets processes at the LHC, Phys. Rev. D 99 (2019) 094025 [arXiv: 1905.00184] [INSPIRE].

[56] A.J. Larkoski, S. Marzani, G. Soyez and J. Thaler, Soft drop, JHEP 05 (2014) 146 [arXiv: 1402.2657] [INSPIRE].

[57] S. Caletti et al., Jet angularities in $Z+$ jet production at the LHC, JHEP 07 (2021) 076 [arXiv: 2104.06920] [INSPIRE].

[58] CMS collaboration, The CMS experiment at the CERN LHC, 2008 JINST 3 S08004 [INSPIRE].

[59] CMS collaboration, Description and performance of track and primary-vertex reconstruction with the CMS tracker, 2014 JINST 9 P10009 [arXiv:1405.6569] [INSPIRE].

[60] M. Cacciari, G.P. Salam and G. Soyez, The anti- $k_{t}$ jet clustering algorithm, JHEP 04 (2008) 063 [arXiv: 0802.1189] [INSPIRE].

[61] M. Cacciari, G.P. Salam and G. Soyez, FastJet user manual, Eur. Phys. J. C 72 (2012) 1896 [arXiv:1111.6097] [INSPIRE].

[62] CMS collaboration, Performance of the CMS muon detector and muon reconstruction with proton-proton collisions at $\sqrt{s}=13 \mathrm{TeV}, 2018$ JINST $13 \mathrm{P} 06015$ [arXiv: 1804.04528] [INSPIRE].

[63] CMS collaboration, Particle-flow reconstruction and global event description with the CMS detector, 2017 JINST 12 P10003 [arXiv: 1706.04965] [INSPIRE].

[64] D. Bertolini, P. Harris, M. Low and N. Tran, Pileup per particle identification, JHEP 10 (2014) 059 [arXiv: 1407.6013] [InSPIRE].

[65] CMS collaboration, Pileup mitigation at CMS in $13 \mathrm{TeV}$ data, 2020 JINST 15 P09018 [arXiv: 2003. 00503] [INSPIRE].

[66] CMS collaboration, Jet energy scale and resolution in the CMS experiment in pp collisions at $8 \mathrm{TeV}, 2017$ JINST 12 P02014 [arXiv: 1607.03663] [INSPIRE].

[67] CMS collaboration, The CMS trigger system, 2017 JINST 12 P01020 [arXiv:1609.02366] [INSPIRE].

[68] CMS collaboration, Performance of the CMS level-1 trigger in proton-proton collisions at $\sqrt{s}=13 \mathrm{TeV}, 2020$ JINST 15 P10017 [arXiv:2006.10165] [INSPIRE].

[69] CMS collaboration, Performance of the CMS muon trigger system in proton-proton collisions at $\sqrt{s}=13 \mathrm{TeV}, 2021$ JINST $16 \mathrm{P} 07001$ [arXiv:2102.04790] [INSPIRE]. 
[70] J. Alwall et al., The automated computation of tree-level and next-to-leading order differential cross sections, and their matching to parton shower simulations, JHEP $\mathbf{0 7}$ (2014) 079 [arXiv: 1405.0301] [INSPIRE].

[71] T. Sjöstrand et al., An introduction to PYTHIA 8.2, Comput. Phys. Commun. 191 (2015) 159 [arXiv: 1410.3012] [INSPIRE].

[72] B. Andersson, G. Gustafson, G. Ingelman and T. Sjöstrand, Parton fragmentation and string dynamics, Phys. Rept. 97 (1983) 31 [inSPIRE].

[73] T. Sjöstrand, The merging of jets, Phys. Lett. B 142 (1984) 420 [INSPIRE].

[74] J. Alwall et al., Comparative study of various algorithms for the merging of parton showers and matrix elements in hadronic collisions, Eur. Phys. J. C 53 (2008) 473 [arXiv:0706.2569] [INSPIRE].

[75] P. Skands, S. Carrazza and J. Rojo, Tuning PYTHIA 8.1: the Monash 2013 tune, Eur. Phys. J. C 74 (2014) 3024 [arXiv: 1404.5630] [InSPIRE].

[76] CMS collaboration, Event generator tunes obtained from underlying event and multiparton scattering measurements, Eur. Phys. J. C 76 (2016) 155 [arXiv:1512.00815] [INSPIRE].

[77] M. Bahr et al., HERWIG++ physics and manual, Eur. Phys. J. C 58 (2008) 639 [arXiv:0803.0883] [INSPIRE].

[78] J. Bellm et al., HERWIG++ 2.7 release note, arXiv:1310.6877 [INSPIRE].

[79] M.H. Seymour and A. Siodmok, Constraining MPI models using $\sigma_{\mathrm{eff}}$ and recent Tevatron and LHC underlying event data, JHEP 10 (2013) 113 [arXiv: 1307.5015] [INSPIRE].

[80] S. Gieseke, P. Stephens and B. Webber, New formalism for QCD parton showers, JHEP 12 (2003) 045 [hep-ph/0310083] [INSPIRE].

[81] B.R. Webber, A QCD model for jet fragmentation including soft gluon interference, Nucl. Phys. B 238 (1984) 492 [INSPIRE].

[82] J.M. Lindert et al., Precise predictions for $V+$ jets dark matter backgrounds, Eur. Phys. J. $C 77$ (2017) 829 [arXiv: 1705.04664] [INSPIRE].

[83] NNPDF collaboration, Parton distributions for the LHC run II, JHEP 04 (2015) 040 [arXiv: 1410.8849] [INSPIRE].

[84] GEANT4 collaboration, GEANT4 - a simulation toolkit, Nucl. Instrum. Meth. A $\mathbf{5 0 6}$ (2003) 250 [INSPIRE].

[85] CMS collaboration, Extraction and validation of a new set of CMS PYTHIA8 tunes from underlying-event measurements, Eur. Phys. J. C 80 (2020) 4 [arXiv:1903.12179] [INSPIRE].

[86] J. Bellm et al., HERWIG 7.0/HERWIG++3.0 release note, Eur. Phys. J. C 76 (2016) 196 [arXiv: 1512.01178] [INSPIRE].

[87] CMS collaboration, Development and validation of HERWIG 7 tunes from CMS underlying-event measurements, Eur. Phys. J. C 81 (2021) 312 [arXiv:2011.03422] [INSPIRE].

[88] Sherpa collaboration, Event generation with Sherpa 2.2, SciPost Phys. 7 (2019) 034 [arXiv: 1905.09127] [INSPIRE].

[89] F. Krauss, R. Kuhn and G. Soff, $A M E G I C++1.0$ : a matrix element generator in $\mathrm{C}++$, JHEP 02 (2002) 044 [hep-ph/0109036] [inSPIRE]. 
[90] T. Gleisberg and S. Hoeche, Comix, a new matrix element generator, JHEP 12 (2008) 039 [arXiv:0808.3674] [INSPIRE].

[91] S. Catani, F. Krauss, R. Kuhn and B.R. Webber, QCD matrix elements + parton showers, JHEP 11 (2001) 063 [hep-ph/0109231] [INSPIRE].

[92] F. Krauss, Matrix elements and parton showers in hadronic interactions, JHEP 08 (2002) 015 [hep-ph/0205283] [INSPIRE].

[93] A. Schalicke and F. Krauss, Implementing the ME+PS merging algorithm, JHEP 07 (2005) 018 [hep-ph/0503281] [INSPIRE].

[94] S. Schumann and F. Krauss, A parton shower algorithm based on Catani-Seymour dipole factorisation, JHEP 03 (2008) 038 [arXiv:0709.1027] [INSPIRE].

[95] J.-C. Winter, F. Krauss and G. Soff, A modified cluster hadronization model, Eur. Phys. J. C 36 (2004) 381 [hep-ph/0311085] [INSPIRE].

[96] D. Reichelt, P. Richardson and A. Siodmok, Improving the simulation of quark and gluon jets with HERWIG 7, Eur. Phys. J. C 77 (2017) 876 [arXiv:1708.01491] [InSPIRE].

[97] M. Rubin, G.P. Salam and S. Sapeta, Giant QCD K-factors beyond NLO, JHEP 09 (2010) 084 [arXiv: 1006.2144] [INSPIRE].

[98] A.J. Larkoski, D. Neill and J. Thaler, Jet shapes with the broadening axis, JHEP 04 (2014) 017 [arXiv: 1401.2158] [INSPIRE].

[99] Y.L. Dokshitzer, G.D. Leder, S. Moretti and B.R. Webber, Better jet clustering algorithms, JHEP 08 (1997) 001 [hep-ph/9707323] [INSPIRE].

[100] M. Dasgupta, A. Fregoso, S. Marzani and G.P. Salam, Towards an understanding of jet substructure, JHEP 09 (2013) 029 [arXiv:1307.0007] [INSPIRE].

[101] J.M. Butterworth, A.R. Davison, M. Rubin and G.P. Salam, Jet substructure as a new Higgs search channel at the LHC, Phys. Rev. Lett. 100 (2008) 242001 [arXiv:0802.2470] [INSPIRE].

[102] HEPData record for this analysis, CMS-SMP-20-010, (2021).

[103] S. Schmitt, TUnfold: an algorithm for correcting migration effects in high energy physics, 2012 JINST 7 T10003 [arXiv:1205.6201] [INSPIRE].

[104] CMS collaboration, Single-particle response in the CMS calorimeters, CMS Physics Analysis Summary CMS-PAS-JME-10-008, CERN, Geneva, Switzerland (2010).

[105] CMS collaboration, Measurement of the inelastic proton-proton cross section at $\sqrt{s}=13 \mathrm{TeV}$, JHEP 07 (2018) 161 [arXiv: 1802.02613] [inSPIRE].

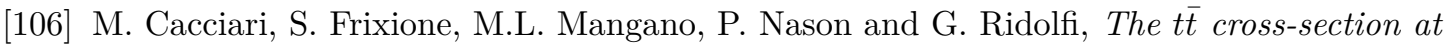
$1.8 \mathrm{TeV}$ and $1.96 \mathrm{TeV}$ : a study of the systematics due to parton densities and scale dependence, JHEP 04 (2004) 068 [hep-ph/0303085] [INSPIRE].

[107] S. Catani, D. de Florian, M. Grazzini and P. Nason, Soft gluon resummation for Higgs boson production at hadron colliders, JHEP 07 (2003) 028 [hep-ph/0306211] [INSPIRE]. 


\section{The CMS collaboration}

\section{Yerevan Physics Institute, Yerevan, Armenia}

A. Tumasyan

\section{Institut für Hochenergiephysik, Vienna, Austria}

W. Adam, J.W. Andrejkovic, T. Bergauer, S. Chatterjee, M. Dragicevic, A. Escalante Del Valle, R. Frühwirth $^{1}$, M. Jeitler ${ }^{1}$, N. Krammer, L. Lechner, D. Liko, I. Mikulec, P. Paulitsch, F.M. Pitters, J. Schieck ${ }^{1}$, R. Schöfbeck, M. Spanring, S. Templ, W. Waltenberger, C.-E. Wulz ${ }^{1}$

Institute for Nuclear Problems, Minsk, Belarus

V. Chekhovsky, A. Litomin, V. Makarenko

Universiteit Antwerpen, Antwerpen, Belgium

M.R. Darwish ${ }^{2}$, E.A. De Wolf, T. Janssen, T. Kello ${ }^{3}$, A. Lelek, H. Rejeb Sfar, P. Van Mechelen, S. Van Putte, N. Van Remortel

\section{Vrije Universiteit Brussel, Brussel, Belgium}

F. Blekman, E.S. Bols, J. D'Hondt, M. Delcourt, H. El Faham, S. Lowette, S. Moortgat, A. Morton, D. Müller, A.R. Sahasransu, S. Tavernier, W. Van Doninck, P. Van Mulders

\section{Université Libre de Bruxelles, Bruxelles, Belgium}

D. Beghin, B. Bilin, B. Clerbaux, G. De Lentdecker, L. Favart, A. Grebenyuk, A.K. Kalsi, K. Lee, M. Mahdavikhorrami, I. Makarenko, L. Moureaux, L. Pétré, A. Popov, N. Postiau, E. Starling, L. Thomas, M. Vanden Bemden, C. Vander Velde, P. Vanlaer, D. Vannerom, L. Wezenbeek

\section{Ghent University, Ghent, Belgium}

T. Cornelis, D. Dobur, J. Knolle, L. Lambrecht, G. Mestdach, M. Niedziela, C. Roskas, A. Samalan, K. Skovpen, M. Tytgat, W. Verbeke, B. Vermassen, M. Vit

\section{Université Catholique de Louvain, Louvain-la-Neuve, Belgium}

A. Bethani, G. Bruno, F. Bury, C. Caputo, P. David, C. Delaere, I.S. Donertas, A. Giammanco, K. Jaffel, Sa. Jain, V. Lemaitre, K. Mondal, J. Prisciandaro, A. Taliercio, M. Teklishyn, T.T. Tran, P. Vischia, S. Wertz

Centro Brasileiro de Pesquisas Fisicas, Rio de Janeiro, Brazil

G.A. Alves, C. Hensel, A. Moraes

\section{Universidade do Estado do Rio de Janeiro, Rio de Janeiro, Brazil}

W.L. Aldá Júnior, M. Alves Gallo Pereira, M. Barroso Ferreira Filho, H. BRANDAO MALBOUISSON, W. Carvalho, J. Chinellato ${ }^{4}$, E.M. Da Costa, G.G. Da Silveira ${ }^{5}$, D. De Jesus Damiao, S. Fonseca De Souza, D. Matos Figueiredo, C. Mora Herrera, K. Mota Amarilo, L. Mundim, H. Nogima, P. Rebello Teles, A. Santoro, S.M. Silva Do Amaral, A. Sznajder, M. Thiel, F. Torres Da Silva De Araujo, A. Vilela Pereira 
Universidade Estadual Paulista ${ }^{a}$, Universidade Federal do $\mathrm{ABC}^{b}$, São Paulo, Brazil

C.A. Bernardes ${ }^{a, a, 5}$, L. Calligaris ${ }^{a}$, T.R. Fernandez Perez Tomei ${ }^{a}$, E.M. Gregores ${ }^{a, b}$, D.S. Lemos ${ }^{a}$, P.G. Mercadante ${ }^{a, b}$, S.F. Novaes ${ }^{a}$, Sandra S. Padula ${ }^{a}$

Institute for Nuclear Research and Nuclear Energy, Bulgarian Academy of Sciences, Sofia, Bulgaria

A. Aleksandrov, G. Antchev, R. Hadjiiska, P. Iaydjiev, M. Misheva, M. Rodozov,

M. Shopova, G. Sultanov

University of Sofia, Sofia, Bulgaria

A. Dimitrov, T. Ivanov, L. Litov, B. Pavlov, P. Petkov, A. Petrov

Beihang University, Beijing, China

T. Cheng, Q. Guo, T. Javaid ${ }^{6}$, M. Mittal, H. Wang, L. Yuan

Department of Physics, Tsinghua University

M. Ahmad, G. Bauer, C. Dozen ${ }^{7}$, Z. Hu, J. Martins ${ }^{8}$, Y. Wang, K. Yi ${ }^{9,10}$

Institute of High Energy Physics, Beijing, China

E. Chapon, G.M. Chen ${ }^{6}$, H.S. Chen ${ }^{6}$, M. Chen, F. Iemmi, A. Kapoor, D. Leggat, H. Liao, Z.-A. LIU ${ }^{6}$, V. Milosevic, F. Monti, R. Sharma, J. Tao, J. Thomas-wilsker, J. Wang, H. Zhang, S. Zhang ${ }^{6}$, J. Zhao

State Key Laboratory of Nuclear Physics and Technology, Peking University, Beijing, China

A. Agapitos, Y. An, Y. Ban, C. Chen, A. Levin, Q. Li, X. Lyu, Y. Mao, S.J. Qian, D. Wang, Q. Wang, J. Xiao

\section{Sun Yat-Sen University, Guangzhou, China}

M. Lu, Z. You

Institute of Modern Physics and Key Laboratory of Nuclear Physics and Ionbeam Application (MOE) — Fudan University, Shanghai, China

X. $\mathrm{Gao}^{3}, \mathrm{H}$. Okawa

Zhejiang University, Hangzhou, China

Z. Lin, M. Xiao

Universidad de Los Andes, Bogota, Colombia

C. Avila, A. Cabrera, C. Florez, J. Fraga

Universidad de Antioquia, Medellin, Colombia

J. Mejia Guisao, F. Ramirez, J.D. Ruiz Alvarez, C.A. Salazar González

University of Split, Faculty of Electrical Engineering, Mechanical Engineering and Naval Architecture, Split, Croatia

D. Giljanovic, N. Godinovic, D. Lelas, I. Puljak 
University of Split, Faculty of Science, Split, Croatia

Z. Antunovic, M. Kovac, T. Sculac

Institute Rudjer Boskovic, Zagreb, Croatia

V. Brigljevic, D. Ferencek, D. Majumder, M. Roguljic, A. Starodumov ${ }^{11}$, T. Susa

University of Cyprus, Nicosia, Cyprus

A. Attikis, K. Christoforou, E. Erodotou, A. Ioannou, G. Kole, M. Kolosova, S. Konstantinou, J. Mousa, C. Nicolaou, F. Ptochos, P.A. Razis, H. Rykaczewski, H. Saka

Charles University, Prague, Czech Republic

M. Finger ${ }^{12}$, M. Finger Jr. ${ }^{12}$, A. Kveton

Escuela Politecnica Nacional, Quito, Ecuador

E. Ayala

Universidad San Francisco de Quito, Quito, Ecuador

E. Carrera Jarrin

Academy of Scientific Research and Technology of the Arab Republic of Egypt, Egyptian Network of High Energy Physics, Cairo, Egypt

H. Abdalla ${ }^{13}$, S. Abu Zeid ${ }^{14}$

Center for High Energy Physics (CHEP-FU), Fayoum University, El-Fayoum, Egypt

M.A. Mahmoud, Y. Mohammed

National Institute of Chemical Physics and Biophysics, Tallinn, Estonia

S. Bhowmik, R.K. Dewanjee, K. Ehataht, M. Kadastik, S. Nandan, C. Nielsen, J. Pata, M. Raidal, L. Tani, C. Veelken

Department of Physics, University of Helsinki, Helsinki, Finland

P. Eerola, L. Forthomme, H. Kirschenmann, K. Osterberg, M. Voutilainen

Helsinki Institute of Physics, Helsinki, Finland

S. Bharthuar, E. Brücken, F. Garcia, J. Havukainen, M.S. Kim, R. Kinnunen, T. Lampén, K. Lassila-Perini, S. Lehti, T. Lindén, M. Lotti, L. Martikainen, M. Myllymäki, J. Ott, H. Siikonen, E. Tuominen, J. Tuominiemi

Lappeenranta University of Technology, Lappeenranta, Finland

P. Luukka, H. Petrow, T. Tuuva

IRFU, CEA, Université Paris-Saclay, Gif-sur-Yvette, France

C. Amendola, M. Besancon, F. Couderc, M. Dejardin, D. Denegri, J.L. Faure, F. Ferri, S. Ganjour, A. Givernaud, P. Gras, G. Hamel de Monchenault, P. Jarry, B. Lenzi, E. Locci, J. Malcles, J. Rander, A. Rosowsky, M.Ö. Sahin, A. Savoy-Navarro ${ }^{15}$, M. Titov, G.B. Yu

Laboratoire Leprince-Ringuet, CNRS/IN2P3, Ecole Polytechnique, Institut Polytechnique de Paris, Palaiseau, France

S. Ahuja, F. Beaudette, M. Bonanomi, A. Buchot Perraguin, P. Busson, A. Cappati, C. Charlot, O. Davignon, B. Diab, G. Falmagne, S. Ghosh, R. Granier de Cassagnac, 
A. Hakimi, I. Kucher, J. Motta, M. Nguyen, C. Ochando, P. Paganini, J. Rembser, R. Salerno, J.B. Sauvan, Y. Sirois, A. Tarabini, A. Zabi, A. Zghiche

\section{Université de Strasbourg, CNRS, IPHC UMR 7178, Strasbourg, France}

J.-L. Agram ${ }^{16}$, J. Andrea, D. Apparu, D. Bloch, G. Bourgatte, J.-M. Brom, E.C. Chabert, C. Collard, D. Darej, J.-C. Fontaine ${ }^{16}$, U. Goerlach, C. Grimault, A.-C. Le Bihan, E. Nibigira, P. Van Hove

\section{Institut de Physique des 2 Infinis de Lyon (IP2I ), Villeurbanne, France}

E. Asilar, S. Beauceron, C. Bernet, G. Boudoul, C. Camen, A. Carle, N. Chanon, D. Contardo, P. Depasse, H. El Mamouni, J. Fay, S. Gascon, M. Gouzevitch, B. Ille, I.B. Laktineh, H. Lattaud, A. Lesauvage, M. Lethuillier, L. Mirabito, S. Perries, K. Shchablo, V. Sordini, L. Torterotot, G. Touquet, M. Vander Donckt, S. Viret

\section{Georgian Technical University, Tbilisi, Georgia}

I. Lomidze, T. Toriashvili ${ }^{17}, \mathrm{Z}$. Tsamalaidze ${ }^{12}$

\section{RWTH Aachen University, I. Physikalisches Institut, Aachen, Germany}

L. Feld, K. Klein, M. Lipinski, D. Meuser, A. Pauls, M.P. Rauch, N. Röwert, J. Schulz, M. Teroerde

\section{RWTH Aachen University, III. Physikalisches Institut A, Aachen, Germany}

A. Dodonova, D. Eliseev, M. Erdmann, P. Fackeldey, B. Fischer, S. Ghosh, T. Hebbeker, K. Hoepfner, F. Ivone, H. Keller, L. Mastrolorenzo, M. Merschmeyer, A. Meyer, G. Mocellin, S. Mondal, S. Mukherjee, D. Noll, A. Novak, T. Pook, A. Pozdnyakov, Y. Rath, H. Reithler, J. Roemer, A. Schmidt, S.C. Schuler, A. Sharma, L. Vigilante, S. Wiedenbeck, S. Zaleski

\section{RWTH Aachen University, III. Physikalisches Institut B, Aachen, Germany}

C. Dziwok, G. Flügge, W. Haj Ahmad ${ }^{18}$, O. Hlushchenko, T. Kress, A. Nowack, C. Pistone, O. Pooth, D. Roy, H. Sert, A. Stahl ${ }^{19}$, T. Ziemons

\section{Deutsches Elektronen-Synchrotron, Hamburg, Germany}

H. Aarup Petersen, M. Aldaya Martin, P. Asmuss, I. Babounikau, S. Baxter, O. Behnke, A. Bermúdez Martínez, S. Bhattacharya, A.A. Bin Anuar, K. Borras ${ }^{20}$, V. Botta, D. Brunner, A. Campbell, A. Cardini, C. Cheng, F. Colombina, S. Consuegra Rodríguez, G. Correia Silva, V. Danilov, L. Didukh, G. Eckerlin, D. Eckstein, L.I. Estevez Banos, O. Filatov, E. Gallo ${ }^{21}$, A. Geiser, A. Giraldi, A. Grohsjean, M. Guthoff, A. Jafari ${ }^{22}$, N.Z. Jomhari, H. Jung, A. Kasem ${ }^{20}$, M. Kasemann, H. Kaveh, C. Kleinwort, D. Krücker, W. Lange, J. Lidrych, K. Lipka, W. Lohmann ${ }^{23}$, R. Mankel, I.-A. Melzer-Pellmann, M. Mendizabal Morentin, J. Metwally, A.B. Meyer, M. Meyer, J. Mnich, A. Mussgiller, Y. Otarid, D. Pérez Adán, D. Pitzl, A. Raspereza, B. Ribeiro Lopes, J. Rübenach, A. Saggio, A. Saibel, M. Savitskyi, M. Scham, V. Scheurer, P. Schütze, C. Schwanenberger ${ }^{21}$, A. Singh, R.E. Sosa Ricardo, D. Stafford, N. Tonon, O. Turkot, M. Van De Klundert, R. Walsh, D. Walter, Y. Wen, K. Wichmann, L. Wiens, C. Wissing, S. Wuchterl 


\section{University of Hamburg, Hamburg, Germany}

R. Aggleton, S. Albrecht, S. Bein, L. Benato, A. Benecke, P. Connor, K. De Leo, M. Eich, F. Feindt, A. Fröhlich, C. Garbers, E. Garutti, P. Gunnellini, J. Haller, A. Hinzmann, G. Kasieczka, R. Klanner, R. Kogler, T. Kramer, V. Kutzner, J. Lange, T. Lange, A. Lobanov, A. Malara, A. Nigamova, K.J. Pena Rodriguez, O. Rieger, P. Schleper, M. Schröder, J. Schwandt, D. Schwarz, J. Sonneveld, H. Stadie, G. Steinbrück, A. Tews, B. Vormwald, I. Zoi

\section{Karlsruher Institut fuer Technologie, Karlsruhe, Germany}

J. Bechtel, T. Berger, E. Butz, R. Caspart, T. Chwalek, W. De Boer ${ }^{\dagger}$, A. Dierlamm, A. Droll, K. El Morabit, N. Faltermann, M. Giffels, J.o. Gosewisch, A. Gottmann, F. Hartmann ${ }^{19}$, C. Heidecker, U. Husemann, P. Keicher, R. Koppenhöfer, S. Maier, M. Metzler, S. Mitra, Th. Müller, M. Neukum, A. Nürnberg, G. Quast, K. Rabbertz, J. Rauser, D. Savoiu, M. Schnepf, D. Seith, I. Shvetsov, H.J. Simonis, R. Ulrich, J. Van Der Linden, R.F. Von Cube, M. Wassmer, M. Weber, S. Wieland, R. Wolf, S. Wozniewski, S. Wunsch

Institute of Nuclear and Particle Physics (INPP), NCSR Demokritos, Aghia Paraskevi, Greece

G. Anagnostou, G. Daskalakis, T. Geralis, A. Kyriakis, D. Loukas, A. Stakia

National and Kapodistrian University of Athens, Athens, Greece

M. Diamantopoulou, D. Karasavvas, G. Karathanasis, P. Kontaxakis, C.K. Koraka, A. Manousakis-katsikakis, A. Panagiotou, I. Papavergou, N. Saoulidou, K. Theofilatos, E. Tziaferi, K. Vellidis, E. Vourliotis

National Technical University of Athens, Athens, Greece

G. Bakas, K. Kousouris, I. Papakrivopoulos, G. Tsipolitis, A. Zacharopoulou

University of Ioánnina, Ioánnina, Greece

I. Evangelou, C. Foudas, P. Gianneios, P. Katsoulis, P. Kokkas, N. Manthos, I. Papadopoulos, J. Strologas

MTA-ELTE Lendület CMS Particle and Nuclear Physics Group, Eötvös Loránd University

M. Csanad, K. Farkas, M.M.A. Gadallah ${ }^{24}$, S. Lökös ${ }^{25}$, P. Major, K. Mandal, A. Mehta, G. Pasztor, A.J. Rádl, O. Surányi, G.I. Veres

Wigner Research Centre for Physics, Budapest, Hungary

M. Bartók ${ }^{26}$, G. Bencze, C. Hajdu, D. Horvath ${ }^{27}$, F. Sikler, V. Veszpremi, G. Vesztergombi ${ }^{\dagger}$

Institute of Nuclear Research ATOMKI, Debrecen, Hungary

S. Czellar, J. Karancsi ${ }^{26}$, J. Molnar, Z. Szillasi, D. Teyssier

Institute of Physics, University of Debrecen

P. Raics, Z.L. Trocsanyi ${ }^{28}$, B. Ujvari

Karoly Robert Campus, MATE Institute of Technology

T. Csorgo ${ }^{29}$, F. Nemes ${ }^{29}$, T. Novak 
Indian Institute of Science (IISc), Bangalore, India

J.R. Komaragiri, D. Kumar, L. Panwar, P.C. Tiwari

National Institute of Science Education and Research, HBNI, Bhubaneswar, India

S. Bahinipati ${ }^{30}$, C. Kar, P. Mal, T. Mishra, V.K. Muraleedharan Nair Bindhu ${ }^{31}$, A. Nayak ${ }^{31}$, P. Saha, N. Sur, S.K. Swain, D. Vats ${ }^{31}$

Panjab University, Chandigarh, India

S. Bansal, S.B. Beri, V. Bhatnagar, G. Chaudhary, S. Chauhan, N. Dhingra ${ }^{32}$, R. Gupta, A. Kaur, M. Kaur, S. Kaur, P. Kumari, M. Meena, K. Sandeep, J.B. Singh, A.K. Virdi

University of Delhi, Delhi, India

A. Ahmed, A. Bhardwaj, B.C. Choudhary, M. Gola, S. Keshri, A. Kumar, M. Naimuddin, P. Priyanka, K. Ranjan, A. Shah

Saha Institute of Nuclear Physics, HBNI, Kolkata, India

M. Bharti ${ }^{33}$, R. Bhattacharya, S. Bhattacharya, D. Bhowmik, S. Dutta, S. Dutta, B. Gomber ${ }^{34}$, M. Maity ${ }^{35}$, P. Palit, P.K. Rout, G. Saha, B. Sahu, S. Sarkar, M. Sharan, B. Singh ${ }^{33}$, S. Thakur ${ }^{33}$

Indian Institute of Technology Madras, Madras, India

P.K. Behera, S.C. Behera, P. Kalbhor, A. Muhammad, R. Pradhan, P.R. Pujahari, A. Sharma, A.K. Sikdar

Bhabha Atomic Research Centre, Mumbai, India

D. Dutta, V. Jha, V. Kumar, D.K. Mishra, K. Naskar ${ }^{36}$, P.K. Netrakanti, L.M. Pant, P. Shukla

Tata Institute of Fundamental Research-A, Mumbai, India

T. Aziz, S. Dugad, M. Kumar, U. Sarkar

Tata Institute of Fundamental Research-B, Mumbai, India

S. Banerjee, R. Chudasama, M. Guchait, S. Karmakar, S. Kumar, G. Majumder, K. Mazumdar, S. Mukherjee

Indian Institute of Science Education and Research (IISER), Pune, India

K. Alpana, S. Dube, B. Kansal, A. Laha, S. Pandey, A. Rane, A. Rastogi, S. Sharma

Isfahan University of Technology, Isfahan, Iran

H. Bakhshiansohi ${ }^{37}$, M. Zeinali ${ }^{38}$

Institute for Research in Fundamental Sciences (IPM), Tehran, Iran

S. Chenarani ${ }^{39}$, S.M. Etesami, M. Khakzad, M. Mohammadi Najafabadi

University College Dublin, Dublin, Ireland

M. Grunewald 
INFN Sezione di Bari ${ }^{a}$, Università di Bari ${ }^{b}$, Politecnico di Bari ${ }^{c}$, Bari, Italy

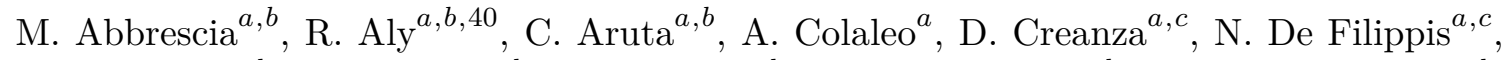
M. De Palma ${ }^{a, b}$, A. Di Florio ${ }^{a, b}$, A. Di Pilato ${ }^{a, b}$, W. Elmetenawee ${ }^{a, b}{\text {, L. } \text { Fiore }^{a} \text {, A. Gelmi }}^{a, b}$,

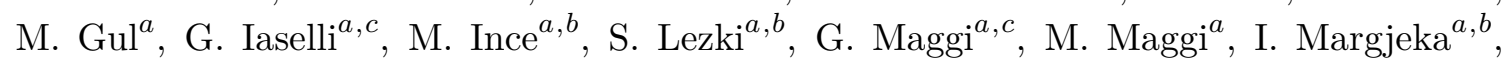
V. Mastrapasqua ${ }^{a, b}$, J.A. Merlin ${ }^{a}$, S. My ${ }^{a, b}$, S. Nuzzo ${ }^{a, b}$, A. Pellecchia ${ }^{a, b}$, A. Pompili ${ }^{a, b}$, G. Pugliese ${ }^{a, c}$, A. Ranieri ${ }^{a}$, G. Selvaggi ${ }^{a, b}$, L. Silvestris ${ }^{a}$, F.M. Simone ${ }^{a, b}$, R. Venditti ${ }^{a}$, P. Verwilligen ${ }^{a}$

INFN Sezione di Bologna ${ }^{a}$, Università di Bologna ${ }^{b}$, Bologna, Italy

G. Abbiendi ${ }^{a}$, C. Battilana ${ }^{a, b}$, D. Bonacorsi ${ }^{a, b}$, L. Borgonovi ${ }^{a}$, L. Brigliadori ${ }^{a}$, R. Campanini ${ }^{a, b}$, P. Capiluppi ${ }^{a, b}$, A. Castro ${ }^{a, b}$, F.R. Cavallo ${ }^{a}$, M. Cuffiani ${ }^{a, b}$, G.M. Dallavalle ${ }^{a}$, T. Diotalevi ${ }^{a, b}$, F. Fabbri ${ }^{a}$, A. Fanfani ${ }^{a, b}$, P. Giacomelli $^{a}$, L. Giommi $^{a, b}{ }^{,}$ C. Grandi ${ }^{a}$, L. Guiducci ${ }^{a, b}$, S. Lo Meo ${ }^{a, 41}$, L. Lunerti ${ }^{a, b}$, S. Marcellini ${ }^{a}$, G. Masetti ${ }^{a}$, F.L. Navarria ${ }^{a, b}$, A. Perrotta ${ }^{a}$, F. Primavera ${ }^{a, b}$, A.M. Rossi ${ }^{a, b}$, T. Rovelli ${ }^{a, b}$, G.P. Siroli ${ }^{a, b}$

INFN Sezione di Catania ${ }^{a}$, Università di Catania ${ }^{b}$, Catania, Italy

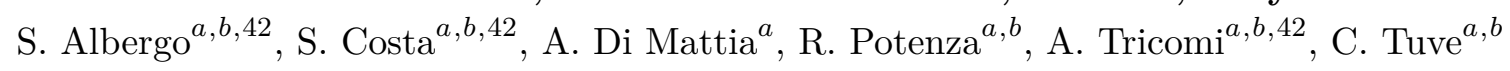
INFN Sezione di Firenze ${ }^{a}$, Università di Firenze ${ }^{b}$, Firenze, Italy

G. Barbagli $^{a}$, A. Cassese ${ }^{a}$, R. Ceccarelli ${ }^{a, b}$, V. Ciulli $^{a, b}$, C. Civinini $^{a}$, R. D'Alessandro ${ }^{a, b}$, E. Focardi ${ }^{a, b}$, G. Latino ${ }^{a, b}$, P. Lenzi ${ }^{a, b}$, M. Lizzo ${ }^{a, b}$, M. Meschini ${ }^{a}$, S. Paoletti ${ }^{a}$, R. Seidita ${ }^{a, b}$, G. Sguazzoni ${ }^{a}$, L. Viliani $^{a}$

INFN Laboratori Nazionali di Frascati, Frascati, Italy

L. Benussi, S. Bianco, D. Piccolo

INFN Sezione di Genova ${ }^{a}$, Università di Genova ${ }^{b}$, Genova, Italy

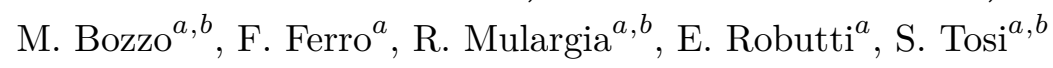

INFN Sezione di Milano-Bicocca ${ }^{a}$, Università di Milano-Bicocca ${ }^{b}$, Milano, Italy

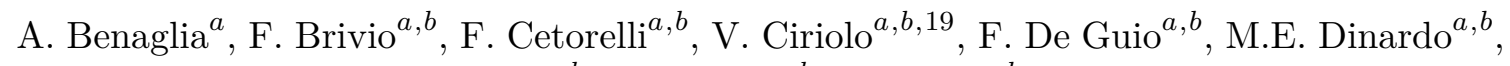
P. Dini ${ }^{a}$, S. Gennai ${ }^{a}$, A. Ghezzi ${ }^{a, b}$, P. Govoni $^{a}, b$, L. Guzzi $^{a}, b$, M. Malberti $^{a}$, S. Malvezzi $^{a}$, A. Massironi ${ }^{a}$, D. Menasce ${ }^{a}$, L. Moroni ${ }^{a}$, M. Paganoni ${ }^{a, b}$, D. Pedrini ${ }^{a}$, S. Ragazzi ${ }^{a, b}$, N. Redaelli ${ }^{a}$, T. Tabarelli de Fatis ${ }^{a, b}$, D. Valsecchi ${ }^{a, b, 19}$, D. Zuolo ${ }^{a, b}$

INFN Sezione di Napoli ${ }^{a}$, Università di Napoli 'Federico II' ${ }^{b}$, Napoli, Italy, Università della Basilicata ${ }^{c}$, Potenza, Italy, Università G. Marconi ${ }^{d}$, Roma, Italy

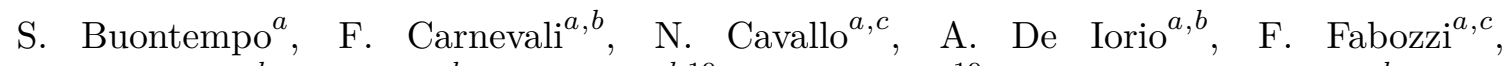

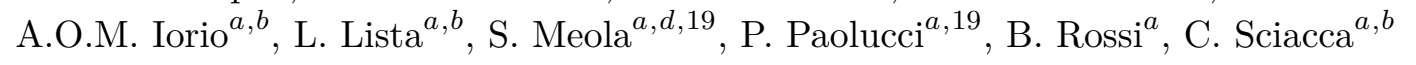

INFN Sezione di Padova ${ }^{a}$, Università di Padova ${ }^{b}$, Padova, Italy, Università di Trento ${ }^{c}$, Trento, Italy

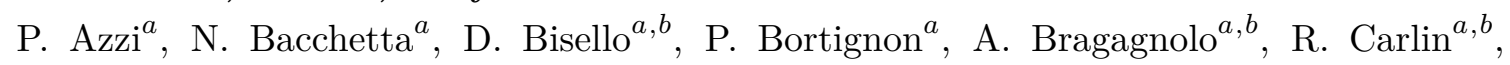

P. Checchia ${ }^{a}$, T. Dorigo ${ }^{a}$, U. Dosselli ${ }^{a}$, F. Gasparini ${ }^{a, b}$, U. Gasparini ${ }^{a, b}$, S.Y. Hoh $^{a, b}$,

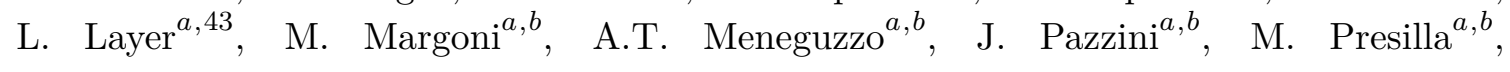




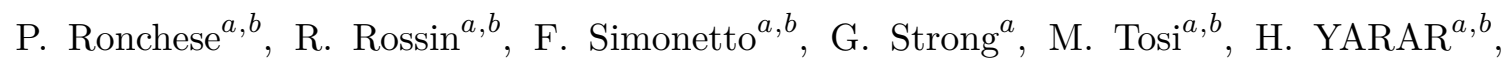
M. Zanetti ${ }^{a, b}$, P. Zotto ${ }^{a, b}$, A. Zucchetta $^{a, b}$, G. Zumerle ${ }^{a, b}$

INFN Sezione di Pavia ${ }^{a}$, Università di Pavia ${ }^{b}$

C. Aime ${ }^{a, b}$, A. Braghieri ${ }^{a}$, S. Calzaferri ${ }^{a, b}$, D. Fiorina ${ }^{a, b}$, P. Montagna ${ }^{a, b}$, S.P. Ratti ${ }^{a, b}$,

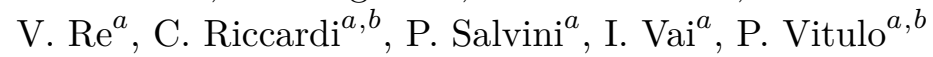

INFN Sezione di Perugia ${ }^{a}$, Università di Perugia ${ }^{b}$, Perugia, Italy

P. Asenov ${ }^{a, 44}$, G.M. Bilei ${ }^{a}$, D. Ciangottini ${ }^{a, b}$, L. Fanò ${ }^{a, b}$, P. Lariccia ${ }^{a, b}$, M. Magherini $^{b}$,

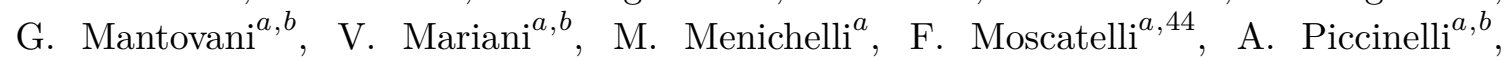

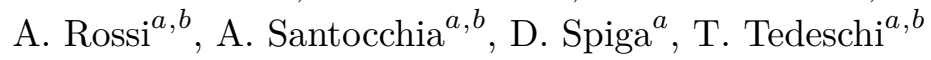

INFN Sezione di Pisa ${ }^{a}$, Università di Pisa ${ }^{b}$, Scuola Normale Superiore di Pisa ${ }^{c}$, Pisa Italy, Università di Siena ${ }^{d}$, Siena, Italy

P. Azzurri ${ }^{a}$, G. Bagliesi ${ }^{a}$, V. Bertacchi ${ }^{a, c}$, L. Bianchini ${ }^{a}$, T. Boccali ${ }^{a}$, E. Bossini ${ }^{a, b}$, R. Castaldi $^{a}$, M.A. Ciocci ${ }^{a, b}$, V. D'Amante ${ }^{a, d}$, R. Dell'Orso ${ }^{a}$, M.R. Di Domenico ${ }^{a, d}$,

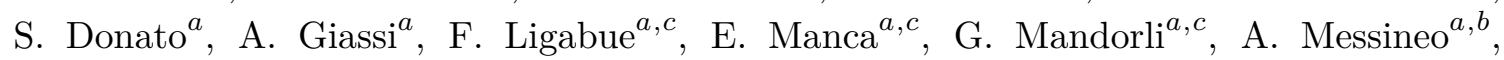
F. Palla ${ }^{a}$, S. Parolia ${ }^{a, b}$, G. Ramirez-Sanchez ${ }^{a, c}$, A. Rizzi ${ }^{a, b}$, G. Rolandi ${ }^{a, c}$, S. Roy Chowdhury ${ }^{a, c}$, A. Scribano ${ }^{a}$, N. Shafiei ${ }^{a, b}$, P. Spagnolo $^{a}$, R. Tenchini ${ }^{a}$, G. Tonelli $^{a, b}{ }$ N. Turini ${ }^{a, d}$, A. Venturi ${ }^{a}$, P.G. Verdini ${ }^{a}$

INFN Sezione di Roma ${ }^{a}$, Sapienza Università di Roma ${ }^{b}$, Rome, Italy M. Campana ${ }^{a, b}$, F. Cavallari ${ }^{a}$, D. Del Re ${ }^{a, b}$, E. Di Marco ${ }^{a}$, M. Diemoz $^{a}$, E. Longo ${ }^{a, b}$, P. Meridiani ${ }^{a}$, G. Organtini ${ }^{a, b}$, F. Pandolfi ${ }^{a}$, R. Paramatti ${ }^{a, b}$, C. Quaranta ${ }^{a, b}$,

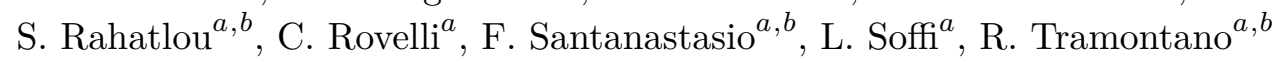

INFN Sezione di Torino ${ }^{a}$, Università di Torino ${ }^{b}$, Torino, Italy, Università del Piemonte Orientale ${ }^{c}$, Novara, Italy

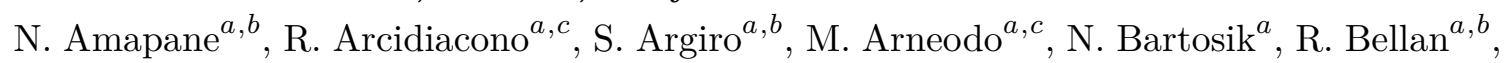

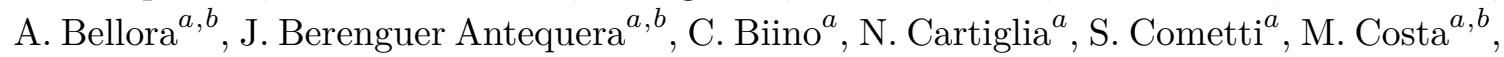
R. Covarelli ${ }^{a, b}$, N. Demaria ${ }^{a}$, B. Kiani ${ }^{a, b}$, F. Legger ${ }^{a}$, C. Mariotti ${ }^{a}$, S. Maselli ${ }^{a}$, E. Migliore $^{a, b}$, E. Monteil ${ }^{a, b}$, M. Monteno ${ }^{a}$, M.M. Obertino ${ }^{a, b}$, G. Ortona $^{a}$, L. Pacher ${ }^{a, b}$, N. Pastrone $^{a}$, M. Pelliccioni ${ }^{a}$, G.L. Pinna Angioni ${ }^{a, b}$, M. Ruspa ${ }^{a, c}$, K. Shchelina ${ }^{a, b}$,

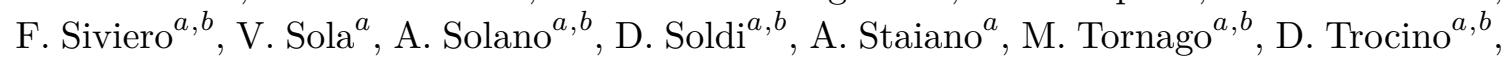
A. Vagnerini

INFN Sezione di Trieste ${ }^{a}$, Università di Trieste ${ }^{b}$, Trieste, Italy

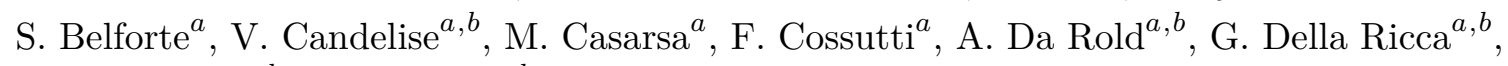
G. Sorrentino $^{a, b}$, F. Vazzoler ${ }^{a, b}$

\section{Kyungpook National University, Daegu, Korea}

S. Dogra, C. Huh, B. Kim, D.H. Kim, G.N. Kim, J. Kim, J. Lee, S.W. Lee, C.S. Moon, Y.D. Oh, S.I. Pak, B.C. Radburn-Smith, S. Sekmen, Y.C. Yang

Chonnam National University, Institute for Universe and Elementary Particles, Kwangju, Korea

H. Kim, D.H. Moon 
Hanyang University, Seoul, Korea

B. Francois, T.J. Kim, J. Park

Korea University, Seoul, Korea

S. Cho, S. Choi, Y. Go, B. Hong, K. Lee, K.S. Lee, J. Lim, J. Park, S.K. Park, J. Yoo

Kyung Hee University, Department of Physics, Seoul, Republic of Korea

J. Goh, A. Gurtu

Sejong University, Seoul, Korea

H.S. Kim, Y. Kim

Seoul National University, Seoul, Korea

J. Almond, J.H. Bhyun, J. Choi, S. Jeon, J. Kim, J.S. Kim, S. Ko, H. Kwon, H. Lee, S. Lee, B.H. Oh, M. Oh, S.B. Oh, H. Seo, U.K. Yang, I. Yoon

University of Seoul, Seoul, Korea

W. Jang, D.Y. Kang, Y. Kang, S. Kim, B. Ko, J.S.H. Lee, Y. Lee, I.C. Park, Y. Roh, M.S. Ryu, D. Song, I.J. Watson, S. Yang

Yonsei University, Department of Physics, Seoul, Korea

S. Ha, H.D. Yoo

Sungkyunkwan University, Suwon, Korea

M. Choi, Y. Jeong, H. Lee, Y. Lee, I. Yu

College of Engineering and Technology, American University of the Middle East (AUM), Egaila, Kuwait

T. Beyrouthy, Y. Maghrbi

Riga Technical University

T. Torims, V. Veckalns ${ }^{45}$

Vilnius University, Vilnius, Lithuania

M. Ambrozas, A. Carvalho Antunes De Oliveira, A. Juodagalvis, A. Rinkevicius, G. Tamulaitis

National Centre for Particle Physics, Universiti Malaya, Kuala Lumpur, Malaysia

N. Bin Norjoharuddeen, W.A.T. Wan Abdullah, M.N. Yusli, Z. Zolkapli

Universidad de Sonora (UNISON), Hermosillo, Mexico

J.F. Benitez, A. Castaneda Hernandez, M. León Coello, J.A. Murillo Quijada, A. Sehrawat, L. Valencia Palomo

Centro de Investigacion y de Estudios Avanzados del IPN, Mexico City, Mexico G. Ayala, H. Castilla-Valdez, E. De La Cruz-Burelo, I. Heredia-De La Cruz ${ }^{46}$, R. LopezFernandez, C.A. Mondragon Herrera, D.A. Perez Navarro, A. Sanchez-Hernandez

Universidad Iberoamericana, Mexico City, Mexico

S. Carrillo Moreno, C. Oropeza Barrera, F. Vazquez Valencia 
Benemerita Universidad Autonoma de Puebla, Puebla, Mexico

I. Pedraza, H.A. Salazar Ibarguen, C. Uribe Estrada

University of Montenegro, Podgorica, Montenegro

J. Mijuskovic ${ }^{47}$, N. Raicevic

University of Auckland, Auckland, New Zealand

D. Krofcheck

University of Canterbury, Christchurch, New Zealand

S. Bheesette, P.H. Butler

National Centre for Physics, Quaid-I-Azam University, Islamabad, Pakistan

A. Ahmad, M.I. Asghar, A. Awais, M.I.M. Awan, H.R. Hoorani, W.A. Khan, M.A. Shah, M. Shoaib, M. Waqas

AGH University of Science and Technology Faculty of Computer Science, Electronics and Telecommunications, Krakow, Poland

V. Avati, L. Grzanka, M. Malawski

National Centre for Nuclear Research, Swierk, Poland

H. Bialkowska, M. Bluj, B. Boimska, M. Górski, M. Kazana, M. Szleper, P. Zalewski

Institute of Experimental Physics, Faculty of Physics, University of Warsaw, Warsaw, Poland

K. Bunkowski, K. Doroba, A. Kalinowski, M. Konecki, J. Krolikowski, M. Walczak

Laboratório de Instrumentação e Física Experimental de Partículas, Lisboa, Portugal

M. Araujo, P. Bargassa, D. Bastos, A. Boletti, P. Faccioli, M. Gallinaro, J. Hollar, N. Leonardo, T. Niknejad, M. Pisano, J. Seixas, O. Toldaiev, J. Varela

Joint Institute for Nuclear Research, Dubna, Russia

S. Afanasiev, D. Budkouski, I. Golutvin, I. Gorbunov, V. Karjavine, V. Korenkov, A. Lanev, A. Malakhov, V. Matveev ${ }^{48,49}$, V. Palichik, V. Perelygin, M. Savina, D. Seitova, V. Shalaev, S. Shmatov, S. Shulha, V. Smirnov, O. Teryaev, N. Voytishin, B.S. Yuldashev ${ }^{50}$, A. Zarubin, I. Zhizhin

Petersburg Nuclear Physics Institute, Gatchina (St. Petersburg), Russia

G. Gavrilov, V. Golovtcov, Y. Ivanov, V. Kim ${ }^{51}$, E. Kuznetsova ${ }^{52}$, V. Murzin, V. Oreshkin, I. Smirnov, D. Sosnov, V. Sulimov, L. Uvarov, S. Volkov, A. Vorobyev

Institute for Nuclear Research, Moscow, Russia

Yu. Andreev, A. Dermenev, S. Gninenko, N. Golubev, A. Karneyeu, D. Kirpichnikov, M. Kirsanov, N. Krasnikov, A. Pashenkov, G. Pivovarov, D. Tlisov ${ }^{\dagger}$, A. Toropin

Institute for Theoretical and Experimental Physics named by A.I. Alikhanov of NRC 'Kurchatov Institute', Moscow, Russia

V. Epshteyn, V. Gavrilov, N. Lychkovskaya, A. Nikitenko ${ }^{53}$, V. Popov, A. Spiridonov, A. Stepennov, M. Toms, E. Vlasov, A. Zhokin 
Moscow Institute of Physics and Technology, Moscow, Russia

T. Aushev

National Research Nuclear University 'Moscow Engineering Physics Institute' (MEPhI), Moscow, Russia

O. Bychkova, M. Chadeeva ${ }^{54}$, P. Parygin, E. Popova, V. Rusinov

P.N. Lebedev Physical Institute, Moscow, Russia

V. Andreev, M. Azarkin, I. Dremin, M. Kirakosyan, A. Terkulov

Skobeltsyn Institute of Nuclear Physics, Lomonosov Moscow State University, Moscow, Russia

A. Belyaev, E. Boos, M. Dubinin ${ }^{55}$, L. Dudko, A. Ershov, A. Gribushin, V. Klyukhin,

O. Kodolova, I. Lokhtin, S. Obraztsov, S. Petrushanko, V. Savrin, A. Snigirev

Novosibirsk State University (NSU), Novosibirsk, Russia

V. Blinov ${ }^{56}$, T. Dimova ${ }^{56}$, L. Kardapoltsev ${ }^{56}$, A. Kozyrev ${ }^{56}$, I. Ovtin ${ }^{56}$, Y. Skovpen ${ }^{56}$

Institute for High Energy Physics of National Research Centre 'Kurchatov Institute', Protvino, Russia

I. Azhgirey, I. Bayshev, D. Elumakhov, V. Kachanov, D. Konstantinov, P. Mandrik,

V. Petrov, R. Ryutin, S. Slabospitskii, A. Sobol, S. Troshin, N. Tyurin, A. Uzunian,

A. Volkov

National Research Tomsk Polytechnic University, Tomsk, Russia

A. Babaev, V. Okhotnikov

Tomsk State University, Tomsk, Russia

V. Borshch, V. Ivanchenko, E. Tcherniaev

University of Belgrade: Faculty of Physics and VINCA Institute of Nuclear Sciences, Belgrade, Serbia

P. Adzic ${ }^{57}$, M. Dordevic, P. Milenovic, J. Milosevic

Centro de Investigaciones Energéticas Medioambientales y Tecnológicas (CIEMAT), Madrid, Spain

M. Aguilar-Benitez, J. Alcaraz Maestre, A. Álvarez Fernández, I. Bachiller, M. Barrio Luna, Cristina F. Bedoya, C.A. Carrillo Montoya, M. Cepeda, M. Cerrada, N. Colino, B. De La Cruz, A. Delgado Peris, J.P. Fernández Ramos, J. Flix, M.C. Fouz, O. Gonzalez Lopez, S. Goy Lopez, J.M. Hernandez, M.I. Josa, J. León Holgado, D. Moran, Á. Navarro Tobar, C. Perez Dengra, A. Pérez-Calero Yzquierdo, J. Puerta Pelayo, I. Redondo, L. Romero, S. Sánchez Navas, L. Urda Gómez, C. Willmott

Universidad Autónoma de Madrid, Madrid, Spain

J.F. de Trocóniz, R. Reyes-Almanza 
Universidad de Oviedo, Instituto Universitario de Ciencias y Tecnologías Espaciales de Asturias (ICTEA), Oviedo, Spain

B. Alvarez Gonzalez, J. Cuevas, C. Erice, J. Fernandez Menendez, S. Folgueras, I. Gonzalez Caballero, J.R. González Fernández, E. Palencia Cortezon, C. Ramón Álvarez, V. Rodríguez Bouza, A. Trapote, N. Trevisani

Instituto de Física de Cantabria (IFCA), CSIC-Universidad de Cantabria, Santander, Spain

J.A. Brochero Cifuentes, I.J. Cabrillo, A. Calderon, J. Duarte Campderros, M. Fernandez, C. Fernandez Madrazo, P.J. Fernández Manteca, A. García Alonso, G. Gomez, C. Martinez Rivero, P. Martinez Ruiz del Arbol, F. Matorras, P. Matorras Cuevas,

J. Piedra Gomez, C. Prieels, T. Rodrigo, A. Ruiz-Jimeno, L. Scodellaro, I. Vila, J.M. Vizan Garcia

\section{University of Colombo, Colombo, Sri Lanka}

MK Jayananda, B. Kailasapathy ${ }^{58}$, D.U.J. Sonnadara, DDC Wickramarathna

University of Ruhuna, Department of Physics, Matara, Sri Lanka

W.G.D. Dharmaratna, K. Liyanage, N. Perera, N. Wickramage

CERN, European Organization for Nuclear Research, Geneva, Switzerland

T.K. Aarrestad, D. Abbaneo, J. Alimena, E. Auffray, G. Auzinger, J. Baechler, P. Baillon ${ }^{\dagger}$, D. Barney, J. Bendavid, M. Bianco, A. Bocci, T. Camporesi, M. Capeans Garrido, G. Cerminara, S.S. Chhibra, M. Cipriani, L. Cristella, D. d'Enterria, A. Dabrowski, A. David, A. De Roeck, M.M. Defranchis, M. Deile, M. Dobson, M. Dünser, N. Dupont, A. ElliottPeisert, N. Emriskova, F. Fallavollita ${ }^{59}$, D. Fasanella, A. Florent, G. Franzoni, W. Funk, S. Giani, D. Gigi, K. Gill, F. Glege, L. Gouskos, M. Haranko, J. Hegeman, Y. Iiyama, V. Innocente, T. James, P. Janot, J. Kaspar, J. Kieseler, M. Komm, N. Kratochwil, C. Lange, S. Laurila, P. Lecoq, K. Long, C. Lourenço, L. Malgeri, S. Mallios, M. Mannelli, A.C. Marini, F. Meijers, S. Mersi, E. Meschi, F. Moortgat, M. Mulders, S. Orfanelli, L. Orsini, F. Pantaleo, L. Pape, E. Perez, M. Peruzzi, A. Petrilli, G. Petrucciani, A. Pfeiffer, M. Pierini, D. Piparo, M. Pitt, H. Qu, T. Quast, D. Rabady, A. Racz, G. Reales Gutiérrez, M. Rieger, M. Rovere, H. Sakulin, J. Salfeld-Nebgen, S. Scarfi, C. Schäfer, C. Schwick, M. Selvaggi, A. Sharma, P. Silva, W. Snoeys, P. Sphicas ${ }^{60}$, S. Summers, K. Tatar, V.R. Tavolaro, D. Treille, A. Tsirou, G.P. Van Onsem, J. Wanczyk ${ }^{61}$, K.A. Wozniak, W.D. Zeuner

Paul Scherrer Institut, Villigen, Switzerland

L. Caminada ${ }^{62}$, A. Ebrahimi, W. Erdmann, R. Horisberger, Q. Ingram, H.C. Kaestli, D. Kotlinski, U. Langenegger, M. Missiroli, T. Rohe

ETH Zurich - Institute for Particle Physics and Astrophysics (IPA), Zurich, Switzerland

K. Androsov ${ }^{61}$, M. Backhaus, P. Berger, A. Calandri, N. Chernyavskaya, A. De Cosa, G. Dissertori, M. Dittmar, M. Donegà, C. Dorfer, F. Eble, K. Gedia, F. Glessgen, T.A. Gómez Espinosa, C. Grab, D. Hits, W. Lustermann, A.-M. Lyon, R.A. Manzoni, 
C. Martin Perez, M.T. Meinhard, F. Nessi-Tedaldi, J. Niedziela, F. Pauss, V. Perovic, S. Pigazzini, M.G. Ratti, M. Reichmann, C. Reissel, T. Reitenspiess, B. Ristic, D. Ruini, D.A. Sanz Becerra, M. Schönenberger, V. Stampf, J. Steggemann ${ }^{61}$, R. Wallny, D.H. Zhu

Universität Zürich, Zurich, Switzerland

C. Amsler ${ }^{63}$, P. Bärtschi, C. Botta, D. Brzhechko, M.F. Canelli, K. Cormier, A. De Wit,

R. Del Burgo, J.K. Heikkilä, M. Huwiler, W. Jin, A. Jofrehei, B. Kilminster, S. Leontsinis, S.P. Liechti, A. Macchiolo, P. Meiring, V.M. Mikuni, U. Molinatti, I. Neutelings, A. Reimers, P. Robmann, S. Sanchez Cruz, K. Schweiger, Y. Takahashi

National Central University, Chung-Li, Taiwan

C. Adloff ${ }^{64}$, C.M. Kuo, W. Lin, A. Roy, T. Sarkar ${ }^{35}$, S.S. Yu

National Taiwan University (NTU), Taipei, Taiwan

L. Ceard, Y. Chao, K.F. Chen, P.H. Chen, W.-S. Hou, Y.y. Li, R.-S. Lu, E. Paganis, A. Psallidas, A. Steen, H.y. Wu, E. Yazgan, P.r. Yu

Chulalongkorn University, Faculty of Science, Department of Physics, Bangkok, Thailand

B. Asavapibhop, C. Asawatangtrakuldee, N. Srimanobhas

Çukurova University, Physics Department, Science and Art Faculty, Adana, Turkey

F. Boran, S. Damarseckin ${ }^{65}$, Z.S. Demiroglu, F. Dolek, I. Dumanoglu ${ }^{66}$, E. Eskut, Y. Guler,

E. Gurpinar Guler ${ }^{67}$, I. Hos ${ }^{68}$, C. Isik, O. Kara, A. Kayis Topaksu, U. Kiminsu, G. Onengut,

K. Ozdemir ${ }^{69}$, A. Polatoz, A.E. Simsek, B. Tali ${ }^{70}$, U.G. Tok, S. Turkcapar, I.S. Zorbakir,

C. Zorbilmez

Middle East Technical University, Physics Department, Ankara, Turkey

B. Isildak ${ }^{71}$, G. Karapinar ${ }^{72}$, K. Ocalan ${ }^{73}$, M. Yalvac ${ }^{74}$

Bogazici University, Istanbul, Turkey

B. Akgun, I.O. Atakisi, E. Gülmez, M. Kaya ${ }^{75}$, O. Kaya ${ }^{76}$, Ö. Özçelik, S. Tekten ${ }^{77}$, E.A. Yetkin ${ }^{78}$

Istanbul Technical University, Istanbul, Turkey

A. Cakir, K. Cankocak ${ }^{66}$, Y. Komurcu, S. Sen ${ }^{79}$

Istanbul University, Istanbul, Turkey

S. $\operatorname{Cerci}^{70}$, B. Kaynak, S. Ozkorucuklu, D. Sunar Cerci ${ }^{70}$

Institute for Scintillation Materials of National Academy of Science of Ukraine, Kharkov, Ukraine

B. Grynyov

National Scientific Center, Kharkov Institute of Physics and Technology, Kharkov, Ukraine

L. Levchuk 


\section{University of Bristol, Bristol, United Kingdom}

D. Anthony, E. Bhal, S. Bologna, J.J. Brooke, A. Bundock, E. Clement, D. Cussans, H. Flacher, J. Goldstein, G.P. Heath, H.F. Heath, M.l. Holmberg ${ }^{80}$, L. Kreczko, B. Krikler, S. Paramesvaran, S. Seif El Nasr-Storey, V.J. Smith, N. Stylianou ${ }^{81}$, K. Walkingshaw Pass, R. White

\section{Rutherford Appleton Laboratory, Didcot, United Kingdom}

K.W. Bell, A. Belyaev ${ }^{82}$, C. Brew, R.M. Brown, D.J.A. Cockerill, C. Cooke, K.V. Ellis, K. Harder, S. Harper, J. Linacre, K. Manolopoulos, D.M. Newbold, E. Olaiya, D. Petyt, T. Reis, T. Schuh, C.H. Shepherd-Themistocleous, I.R. Tomalin, T. Williams

\section{Imperial College, London, United Kingdom}

R. Bainbridge, P. Bloch, S. Bonomally, J. Borg, S. Breeze, O. Buchmuller, V. Cepaitis, G.S. Chahal ${ }^{83}$, D. Colling, P. Dauncey, G. Davies, M. Della Negra, S. Fayer, G. Fedi, G. Hall, M.H. Hassanshahi, G. Iles, J. Langford, L. Lyons, A.-M. Magnan, S. Malik, A. Martelli, D.G. Monk, J. Nash ${ }^{84}$, M. Pesaresi, D.M. Raymond, A. Richards, A. Rose, E. Scott, C. Seez, A. Shtipliyski, A. Tapper, K. Uchida, T. Virdee ${ }^{19}$, M. Vojinovic, N. Wardle, S.N. Webb, D. Winterbottom, A.G. Zecchinelli

\section{Brunel University, Uxbridge, United Kingdom}

K. Coldham, J.E. Cole, A. Khan, P. Kyberd, I.D. Reid, L. Teodorescu, S. Zahid

Baylor University, Waco, U.S.A.

S. Abdullin, A. Brinkerhoff, B. Caraway, J. Dittmann, K. Hatakeyama, A.R. Kanuganti, B. McMaster, N. Pastika, M. Saunders, S. Sawant, C. Sutantawibul, J. Wilson

Catholic University of America, Washington, DC, U.S.A.

R. Bartek, A. Dominguez, R. Uniyal, A.M. Vargas Hernandez

The University of Alabama, Tuscaloosa, U.S.A.

A. Buccilli, S.I. Cooper, D. Di Croce, S.V. Gleyzer, C. Henderson, C.U. Perez, P. Rumerio ${ }^{85}$, C. West

Boston University, Boston, U.S.A.

A. Akpinar, A. Albert, D. Arcaro, C. Cosby, Z. Demiragli, E. Fontanesi, D. Gastler, J. Rohlf, K. Salyer, D. Sperka, D. Spitzbart, I. Suarez, A. Tsatsos, S. Yuan, D. Zou

\section{Brown University, Providence, U.S.A.}

G. Benelli, B. Burkle, X. Coubez ${ }^{20}$, D. Cutts, M. Hadley, U. Heintz, J.M. Hogan ${ }^{86}$, G. Landsberg, K.T. Lau, M. Lukasik, J. Luo, M. Narain, S. Sagir ${ }^{87}$, E. Usai, W.Y. Wong, X. Yan, D. Yu, W. Zhang

University of California, Davis, Davis, U.S.A.

J. Bonilla, C. Brainerd, R. Breedon, M. Calderon De La Barca Sanchez, M. Chertok, J. Conway, P.T. Cox, R. Erbacher, G. Haza, F. Jensen, O. Kukral, R. Lander, M. Mulhearn, D. Pellett, B. Regnery, D. Taylor, Y. Yao, F. Zhang 
University of California, Los Angeles, U.S.A.

M. Bachtis, R. Cousins, A. Datta, D. Hamilton, J. Hauser, M. Ignatenko, M.A. Iqbal, T. Lam, W.A. Nash, S. Regnard, D. Saltzberg, B. Stone, V. Valuev

University of California, Riverside, Riverside, U.S.A.

K. Burt, Y. Chen, R. Clare, J.W. Gary, M. Gordon, G. Hanson, G. Karapostoli, O.R. Long, N. Manganelli, M. Olmedo Negrete, W. Si, S. Wimpenny, Y. Zhang

University of California, San Diego, La Jolla, U.S.A.

J.G. Branson, P. Chang, S. Cittolin, S. Cooperstein, N. Deelen, D. Diaz, J. Duarte, R. Gerosa, L. Giannini, D. Gilbert, J. Guiang, R. Kansal, V. Krutelyov, R. Lee, J. Letts, M. Masciovecchio, S. May, M. Pieri, B.V. Sathia Narayanan, V. Sharma, M. Tadel, A. Vartak, F. Würthwein, Y. Xiang, A. Yagil

University of California, Santa Barbara - Department of Physics, Santa Barbara, U.S.A.

N. Amin, C. Campagnari, M. Citron, A. Dorsett, V. Dutta, J. Incandela, M. Kilpatrick, J. Kim, B. Marsh, H. Mei, M. Oshiro, M. Quinnan, J. Richman, U. Sarica, F. Setti, J. Sheplock, D. Stuart, S. Wang

California Institute of Technology, Pasadena, U.S.A.

A. Bornheim, O. Cerri, I. Dutta, J.M. Lawhorn, N. Lu, J. Mao, H.B. Newman, T.Q. Nguyen, M. Spiropulu, J.R. Vlimant, C. Wang, S. Xie, Z. Zhang, R.Y. Zhu

Carnegie Mellon University, Pittsburgh, U.S.A.

J. Alison, S. An, M.B. Andrews, P. Bryant, T. Ferguson, A. Harilal, C. Liu, T. Mudholkar, M. Paulini, A. Sanchez, W. Terrill

University of Colorado Boulder, Boulder, U.S.A.

J.P. Cumalat, W.T. Ford, A. Hassani, E. MacDonald, R. Patel, A. Perloff, C. Savard, K. Stenson, K.A. Ulmer, S.R. Wagner

Cornell University, Ithaca, U.S.A.

J. Alexander, S. Bright-thonney, Y. Cheng, D.J. Cranshaw, S. Hogan, J. Monroy, J.R. Patterson, D. Quach, J. Reichert, M. Reid, A. Ryd, W. Sun, J. Thom, P. Wittich, R. Zou

Fermi National Accelerator Laboratory, Batavia, U.S.A.

M. Albrow, M. Alyari, G. Apollinari, A. Apresyan, A. Apyan, S. Banerjee, L.A.T. Bauerdick, D. Berry, J. Berryhill, P.C. Bhat, K. Burkett, J.N. Butler, A. Canepa, G.B. Cerati, H.W.K. Cheung, F. Chlebana, M. Cremonesi, K.F. Di Petrillo, V.D. Elvira, Y. Feng, J. Freeman, Z. Gecse, L. Gray, D. Green, S. Grünendahl, O. Gutsche, R.M. Harris, R. Heller, T.C. Herwig, J. Hirschauer, B. Jayatilaka, S. Jindariani, M. Johnson, U. Joshi, T. Klijnsma, B. Klima, K.H.M. Kwok, S. Lammel, D. Lincoln, R. Lipton, T. Liu, C. Madrid, K. Maeshima, C. Mantilla, D. Mason, P. McBride, P. Merkel, S. Mrenna, S. Nahn, J. Ngadiuba, V. O'Dell, V. Papadimitriou, K. Pedro, C. Pena ${ }^{55}$, O. Prokofyev, F. Ravera, A. Reinsvold Hall, L. Ristori, B. Schneider, E. Sexton-Kennedy, N. Smith, A. Soha, W.J. Spalding, L. Spiegel, S. Stoynev, J. Strait, L. Taylor, S. Tkaczyk, N.V. Tran, L. Uplegger, E.W. Vaandering, H.A. Weber 
University of Florida, Gainesville, U.S.A.

D. Acosta, P. Avery, D. Bourilkov, L. Cadamuro, V. Cherepanov, F. Errico, R.D. Field,

D. Guerrero, B.M. Joshi, M. Kim, E. Koenig, J. Konigsberg, A. Korytov, K.H. Lo,

K. Matchev, N. Menendez, G. Mitselmakher, A. Muthirakalayil Madhu, N. Rawal,

D. Rosenzweig, S. Rosenzweig, K. Shi, J. Sturdy, J. Wang, E. Yigitbasi, X. Zuo

Florida State University, Tallahassee, U.S.A.

T. Adams, A. Askew, R. Habibullah, V. Hagopian, K.F. Johnson, R. Khurana, T. Kolberg,

G. Martinez, H. Prosper, C. Schiber, O. Viazlo, R. Yohay, J. Zhang

Florida Institute of Technology, Melbourne, U.S.A.

M.M. Baarmand, S. Butalla, T. Elkafrawy ${ }^{14}$, M. Hohlmann, R. Kumar Verma, D. Noonan, M. Rahmani, F. Yumiceva

University of Illinois at Chicago (UIC), Chicago, U.S.A.

M.R. Adams, H. Becerril Gonzalez, R. Cavanaugh, X. Chen, S. Dittmer, O. Evdokimov, C.E. Gerber, D.A. Hangal, D.J. Hofman, A.H. Merrit, C. Mills, G. Oh, T. Roy, S. Rudrabhatla, M.B. Tonjes, N. Varelas, J. Viinikainen, X. Wang, Z. Wu, Z. Ye

The University of Iowa, Iowa City, U.S.A.

M. Alhusseini, K. Dilsiz ${ }^{88}$, R.P. Gandrajula, O.K. Köseyan, J.-P. Merlo, A. Mestvirishvili ${ }^{89}$, J. Nachtman, H. Ogul ${ }^{90}$, Y. Onel, A. Penzo, C. Snyder, E. Tiras ${ }^{91}$

Johns Hopkins University, Baltimore, U.S.A.

O. Amram, B. Blumenfeld, L. Corcodilos, J. Davis, M. Eminizer, A.V. Gritsan, S. Kyriacou, P. Maksimovic, J. Roskes, M. Swartz, T.Á. Vámi

The University of Kansas, Lawrence, U.S.A.

A. Abreu, J. Anguiano, C. Baldenegro Barrera, P. Baringer, A. Bean, A. Bylinkin, Z. Flowers, T. Isidori, S. Khalil, J. King, G. Krintiras, A. Kropivnitskaya, M. Lazarovits, C. Lindsey, J. Marquez, N. Minafra, M. Murray, M. Nickel, C. Rogan, C. Royon, R. Salvatico, S. Sanders, E. Schmitz, C. Smith, J.D. Tapia Takaki, Q. Wang, Z. Warner, J. Williams, G. Wilson

Kansas State University, Manhattan, U.S.A.

S. Duric, A. Ivanov, K. Kaadze, D. Kim, Y. Maravin, T. Mitchell, A. Modak, K. Nam

Lawrence Livermore National Laboratory, Livermore, U.S.A.

F. Rebassoo, D. Wright

University of Maryland, College Park, U.S.A.

E. Adams, A. Baden, O. Baron, A. Belloni, S.C. Eno, N.J. Hadley, S. Jabeen, R.G. Kellogg, T. Koeth, A.C. Mignerey, S. Nabili, C. Palmer, M. Seidel, A. Skuja, L. Wang, K. Wong

Massachusetts Institute of Technology, Cambridge, U.S.A.

D. Abercrombie, G. Andreassi, R. Bi, S. Brandt, W. Busza, I.A. Cali, Y. Chen, M. D'Alfonso, J. Eysermans, C. Freer, G. Gomez Ceballos, M. Goncharov, P. Harris, M. Hu, M. Klute, D. Kovalskyi, J. Krupa, Y.-J. Lee, B. Maier, C. Mironov, C. Paus, D. Rankin, C. Roland, G. Roland, Z. Shi, G.S.F. Stephans, J. Wang, Z. Wang, B. Wyslouch 
University of Minnesota, Minneapolis, U.S.A.

R.M. Chatterjee, A. Evans, P. Hansen, J. Hiltbrand, Sh. Jain, M. Krohn, Y. Kubota, J. Mans, M. Revering, R. Rusack, R. Saradhy, N. Schroeder, N. Strobbe, M.A. Wadud

University of Nebraska-Lincoln, Lincoln, U.S.A.

K. Bloom, M. Bryson, S. Chauhan, D.R. Claes, C. Fangmeier, L. Finco, F. Golf, C. Joo, I. Kravchenko, M. Musich, I. Reed, J.E. Siado, G.R. Snow ${ }^{\dagger}$, W. Tabb, F. Yan

State University of New York at Buffalo, Buffalo, U.S.A.

G. Agarwal, H. Bandyopadhyay, L. Hay, I. Iashvili, A. Kharchilava, C. McLean, D. Nguyen, J. Pekkanen, S. Rappoccio, A. Williams

Northeastern University, Boston, U.S.A.

G. Alverson, E. Barberis, Y. Haddad, A. Hortiangtham, J. Li, G. Madigan, B. Marzocchi, D.M. Morse, V. Nguyen, T. Orimoto, A. Parker, L. Skinnari, A. Tishelman-Charny, T. Wamorkar, B. Wang, A. Wisecarver, D. Wood

Northwestern University, Evanston, U.S.A.

S. Bhattacharya, J. Bueghly, Z. Chen, A. Gilbert, T. Gunter, K.A. Hahn, Y. Liu, N. Odell, M.H. Schmitt, M. Velasco

University of Notre Dame, Notre Dame, U.S.A.

R. Band, R. Bucci, A. Das, N. Dev, R. Goldouzian, M. Hildreth, K. Hurtado Anampa, C. Jessop, K. Lannon, J. Lawrence, N. Loukas, D. Lutton, N. Marinelli, I. Mcalister, T. McCauley, C. Mcgrady, F. Meng, K. Mohrman, Y. Musienko ${ }^{48}$, R. Ruchti, P. Siddireddy, A. Townsend, M. Wayne, A. Wightman, M. Wolf, M. Zarucki, L. Zygala

The Ohio State University, Columbus, U.S.A.

B. Bylsma, B. Cardwell, L.S. Durkin, B. Francis, C. Hill, M. Nunez Ornelas, K. Wei, B.L. Winer, B.R. Yates

Princeton University, Princeton, U.S.A.

F.M. Addesa, B. Bonham, P. Das, G. Dezoort, P. Elmer, A. Frankenthal, B. Greenberg, N. Haubrich, S. Higginbotham, A. Kalogeropoulos, G. Kopp, S. Kwan, D. Lange, M.T. Lucchini, D. Marlow, K. Mei, I. Ojalvo, J. Olsen, D. Stickland, C. Tully

University of Puerto Rico, Mayaguez, U.S.A.

S. Malik, S. Norberg

Purdue University, West Lafayette, U.S.A.

A.S. Bakshi, V.E. Barnes, R. Chawla, S. Das, L. Gutay, M. Jones, A.W. Jung, S. Karmarkar, M. Liu, G. Negro, N. Neumeister, G. Paspalaki, C.C. Peng, S. Piperov, A. Purohit, J.F. Schulte, M. Stojanovic ${ }^{15}$, J. Thieman, F. Wang, R. Xiao, W. Xie

Purdue University Northwest, Hammond, U.S.A.

J. Dolen, N. Parashar 
Rice University, Houston, U.S.A.

A. Baty, M. Decaro, S. Dildick, K.M. Ecklund, S. Freed, P. Gardner, F.J.M. Geurts, A. Kumar, W. Li, B.P. Padley, R. Redjimi, W. Shi, A.G. Stahl Leiton, S. Yang, L. Zhang, Y. Zhang

University of Rochester, Rochester, U.S.A.

A. Bodek, P. de Barbaro, R. Demina, J.L. Dulemba, C. Fallon, T. Ferbel, M. Galanti, A. Garcia-Bellido, O. Hindrichs, A. Khukhunaishvili, E. Ranken, R. Taus

Rutgers, The State University of New Jersey, Piscataway, U.S.A.

B. Chiarito, J.P. Chou, A. Gandrakota, Y. Gershtein, E. Halkiadakis, A. Hart, M. Heindl, O. Karacheban ${ }^{23}$, I. Laflotte, A. Lath, R. Montalvo, K. Nash, M. Osherson, S. Salur, S. Schnetzer, S. Somalwar, R. Stone, S.A. Thayil, S. Thomas, H. Wang

University of Tennessee, Knoxville, U.S.A.

H. Acharya, A.G. Delannoy, S. Fiorendi, S. Spanier

Texas A\&M University, College Station, U.S.A.

O. Bouhali ${ }^{92}$, M. Dalchenko, A. Delgado, R. Eusebi, J. Gilmore, T. Huang, T. Kamon ${ }^{93}$, H. Kim, S. Luo, S. Malhotra, R. Mueller, D. Overton, D. Rathjens, A. Safonov

Texas Tech University, Lubbock, U.S.A.

N. Akchurin, J. Damgov, V. Hegde, S. Kunori, K. Lamichhane, S.W. Lee, T. Mengke, S. Muthumuni, T. Peltola, I. Volobouev, Z. Wang, A. Whitbeck

Vanderbilt University, Nashville, U.S.A.

E. Appelt, S. Greene, A. Gurrola, W. Johns, A. Melo, H. Ni, K. Padeken, F. Romeo, P. Sheldon, S. Tuo, J. Velkovska

University of Virginia, Charlottesville, U.S.A.

M.W. Arenton, B. Cox, G. Cummings, J. Hakala, R. Hirosky, M. Joyce, A. Ledovskoy, A. Li, C. Neu, B. Tannenwald, S. White, E. Wolfe

Wayne State University, Detroit, U.S.A.

N. Poudyal

University of Wisconsin - Madison, Madison, WI, U.S.A.

K. Black, T. Bose, C. Caillol, S. Dasu, I. De Bruyn, P. Everaerts, F. Fienga, C. Galloni, H. He, M. Herndon, A. Hervé, U. Hussain, A. Lanaro, A. Loeliger, R. Loveless, J. Madhusudanan Sreekala, A. Mallampalli, A. Mohammadi, D. Pinna, A. Savin, V. Shang, V. Sharma, W.H. Smith, D. Teague, S. Trembath-reichert, W. Vetens

$\dagger$ : Deceased

1: Also at TU Wien, Wien, Austria

2: Also at Institute of Basic and Applied Sciences, Faculty of Engineering, Arab Academy for Science, Technology and Maritime Transport, Alexandria, Egypt

3: Also at Université Libre de Bruxelles, Bruxelles, Belgium

4: Also at Universidade Estadual de Campinas, Campinas, Brazil

5: Also at Federal University of Rio Grande do Sul, Porto Alegre, Brazil 
6: Also at University of Chinese Academy of Sciences, Beijing, China

7: Also at Department of Physics, Tsinghua University, Beijing, China

8: Also at UFMS, Nova Andradina, Brazil

9: Also at Nanjing Normal University Department of Physics, Nanjing, China

10: Now at The University of Iowa, Iowa City, U.S.A.

11: Also at Institute for Theoretical and Experimental Physics named by A.I. Alikhanov of NRC 'Kurchatov Institute', Moscow, Russia

12: Also at Joint Institute for Nuclear Research, Dubna, Russia

13: Also at Cairo University, Cairo, Egypt

14: Also at Ain Shams University, Cairo, Egypt

15: Also at Purdue University, West Lafayette, U.S.A.

16: Also at Université de Haute Alsace, Mulhouse, France

17: Also at Tbilisi State University, Tbilisi, Georgia

18: Also at Erzincan Binali Yildirim University, Erzincan, Turkey

19: Also at CERN, European Organization for Nuclear Research, Geneva, Switzerland

20: Also at RWTH Aachen University, III. Physikalisches Institut A, Aachen, Germany

21: Also at University of Hamburg, Hamburg, Germany

22: Also at Isfahan University of Technology, Isfahan, Iran, Isfahan, Iran

23: Also at Brandenburg University of Technology, Cottbus, Germany

24: Also at Physics Department, Faculty of Science, Assiut University, Assiut, Egypt

25: Also at Karoly Robert Campus, MATE Institute of Technology, Gyongyos, Hungary

26: Also at Institute of Physics, University of Debrecen, Debrecen, Hungary

27: Also at Institute of Nuclear Research ATOMKI, Debrecen, Hungary

28: Also at MTA-ELTE Lendület CMS Particle and Nuclear Physics Group, Eötvös Loránd University, Budapest, Hungary

29: Also at Wigner Research Centre for Physics, Budapest, Hungary

30: Also at IIT Bhubaneswar, Bhubaneswar, India

31: Also at Institute of Physics, Bhubaneswar, India

32: Also at G.H.G. Khalsa College, Punjab, India

33: Also at Shoolini University, Solan, India

34: Also at University of Hyderabad, Hyderabad, India

35: Also at University of Visva-Bharati, Santiniketan, India

36: Also at Indian Institute of Technology (IIT), Mumbai, India

37: Also at Deutsches Elektronen-Synchrotron, Hamburg, Germany

38: Also at Sharif University of Technology, Tehran, Iran

39: Also at Department of Physics, University of Science and Technology of Mazandaran, Behshahr, Iran

40: Now at INFN Sezione di Bari, Università di Bari, Politecnico di Bari, Bari, Italy

41: Also at Italian National Agency for New Technologies, Energy and Sustainable Economic Development, Bologna, Italy

42: Also at Centro Siciliano di Fisica Nucleare e di Struttura Della Materia, Catania, Italy

43: Also at Università di Napoli 'Federico II', Napoli, Italy

44: Also at Consiglio Nazionale delle Ricerche - Istituto Officina dei Materiali, Perugia, Italy

45: Also at Riga Technical University, Riga, Latvia

46: Also at Consejo Nacional de Ciencia y Tecnología, Mexico City, Mexico

47: Also at IRFU, CEA, Université Paris-Saclay, Gif-sur-Yvette, France

48: Also at Institute for Nuclear Research, Moscow, Russia

49: Now at National Research Nuclear University 'Moscow Engineering Physics Institute' (MEPhI), Moscow, Russia 
50: Also at Institute of Nuclear Physics of the Uzbekistan Academy of Sciences, Tashkent, Uzbekistan

51: Also at St. Petersburg State Polytechnical University, St. Petersburg, Russia

52: Also at University of Florida, Gainesville, U.S.A.

53: Also at Imperial College, London, United Kingdom

54: Also at P.N. Lebedev Physical Institute, Moscow, Russia

55: Also at California Institute of Technology, Pasadena, U.S.A.

56: Also at Budker Institute of Nuclear Physics, Novosibirsk, Russia

57: Also at Faculty of Physics, University of Belgrade, Belgrade, Serbia

58: Also at Trincomalee Campus, Eastern University, Sri Lanka, Nilaveli, Sri Lanka

59: Also at INFN Sezione di Pavia, Università di Pavia, Pavia, Italy

60: Also at National and Kapodistrian University of Athens, Athens, Greece

61: Also at Ecole Polytechnique Fédérale Lausanne, Lausanne, Switzerland

62: Also at Universität Zürich, Zurich, Switzerland

63: Also at Stefan Meyer Institute for Subatomic Physics, Vienna, Austria

64: Also at Laboratoire d'Annecy-le-Vieux de Physique des Particules, IN2P3-CNRS, Annecyle-Vieux, France

65: Also at Şırnak University, Sirnak, Turkey

66: Also at Near East University, Research Center of Experimental Health Science, Nicosia, Turkey

67: Also at Konya Technical University, Konya, Turkey

68: Also at Istanbul University - Cerrahpasa, Faculty of Engineering, Istanbul, Turkey

69: Also at Piri Reis University, Istanbul, Turkey

70: Also at Adiyaman University, Adiyaman, Turkey

71: Also at Ozyegin University, Istanbul, Turkey

72: Also at Izmir Institute of Technology, Izmir, Turkey

73: Also at Necmettin Erbakan University, Konya, Turkey

74: Also at Bozok Universitetesi Rektörlügü, Yozgat, Turkey

75: Also at Marmara University, Istanbul, Turkey

76: Also at Milli Savunma University, Istanbul, Turkey

77: Also at Kafkas University, Kars, Turkey

78: Also at Istanbul Bilgi University, Istanbul, Turkey

79: Also at Hacettepe University, Ankara, Turkey

80: Also at Rutherford Appleton Laboratory, Didcot, United Kingdom

81: Also at Vrije Universiteit Brussel, Brussel, Belgium

82: Also at School of Physics and Astronomy, University of Southampton, Southampton, United Kingdom

83: Also at IPPP Durham University, Durham, United Kingdom

84: Also at Monash University, Faculty of Science, Clayton, Australia

85: Also at Università di Torino, Torino, Italy

86: Also at Bethel University, St. Paul, Minneapolis/St. Paul, U.S.A.

87: Also at Karamanoğlu Mehmetbey University, Karaman, Turkey

88: Also at Bingol University, Bingol, Turkey

89: Also at Georgian Technical University, Tbilisi, Georgia

90: Also at Sinop University, Sinop, Turkey

91: Also at Erciyes University, Kayseri, Turkey

92: Also at Texas A\&M University at Qatar, Doha, Qatar

93: Also at Kyungpook National University, Daegu, Korea 